Florida International University FIU Digital Commons

3-24-2015

\title{
Comparison of Some Improved Estimators for Linear Regression Model under Different Conditions
}

Smit Shah

Florida International University, sshah060@fiu.edu

DOI: $10.25148 /$ etd.FI15032146

Follow this and additional works at: https://digitalcommons.fiu.edu/etd

Part of the Statistics and Probability Commons

\section{Recommended Citation}

Shah, Smit, "Comparison of Some Improved Estimators for Linear Regression Model under Different Conditions" (2015). FIU

Electronic Theses and Dissertations. 1853.

https://digitalcommons.fiu.edu/etd/1853 
FLORIDA INTERNATIONAL UNIVERSITY

\author{
Miami, Florida
}

COMPARISON OF SOME IMPROVED ESTIMATORS FOR LINEAR REGRESSION MODEL UNDER DIFFERENT CONDITIONS

\author{
A thesis submitted in partial fulfillment of \\ the requirements for the degree of \\ MASTER OF SCIENCE \\ in \\ STATISTICS \\ by \\ Smit Nailesh Shah
}


To: Dean Michael R. Heithaus

College of Arts and Sciences

This thesis, written by Smit Nailesh Shah, and entitled Comparison of some Improved Estimators for Linear Regression Model under Different Conditions, having been approved in respect to style and intellectual content, is referred to you for judgment.

We have read this thesis and recommend that it be approved.

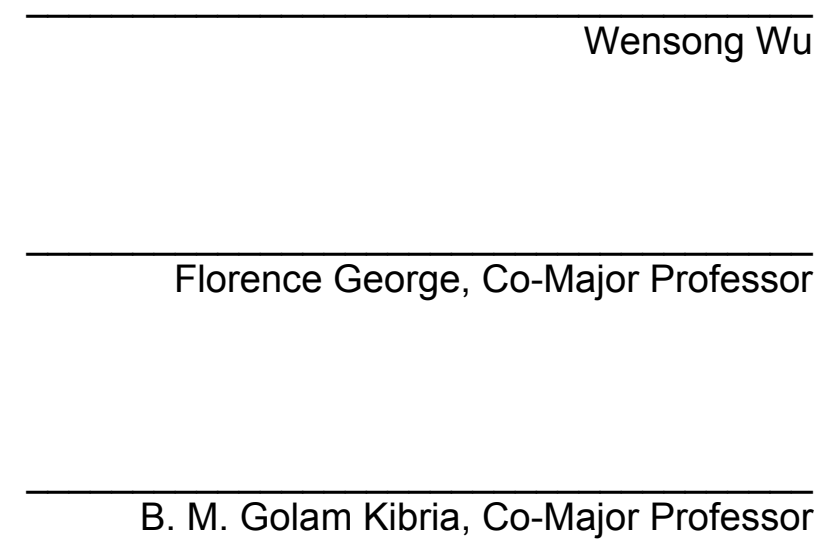

Date of Defense: March 24, 2015

The thesis of Smit Nailesh Shah is approved.

Dean Michael R. Heithaus College of Arts and Sciences

Dean Lakshmi N. Reddi University Graduate School

Florida International University, 2015 


\title{
ABSTRACT OF THE THESIS \\ COMPARISON OF SOME IMPROVED ESTIMATORS FOR LINEAR REGRESSION MODEL UNDER DIFFERENT CONDITIONS
}

\author{
by \\ Smit Nailesh Shah \\ Florida International University, 2015 \\ Miami, Florida \\ Professor B. M. Golam Kibria, Co-Major Professor \\ Professor Florence George, Co-Major Professor
}

Multiple linear regression model plays a key role in statistical inference and it has extensive applications in business, environmental, physical and social sciences. Multicollinearity has been a considerable problem in multiple regression analysis. When the regressor variables are multicollinear, it becomes difficult to make precise statistical inferences about the regression coefficients. There are some statistical methods that can be used, which are discussed in this thesis are ridge regression, Liu, two parameter biased and LASSO estimators. Firstly, an analytical comparison on the basis of risk was made among ridge, Liu and LASSO estimators under orthonormal regression model. I found that LASSO dominates least squares, ridge and Liu estimators over a significant portion of the parameter space for large dimension. Secondly, a simulation study was conducted to compare performance of ridge, Liu and two parameter biased estimator by their mean squared error criterion. I found that two parameter biased estimator performs better than its corresponding ridge regression estimator. Overall, Liu estimator performs better than both ridge and two parameter biased estimator. 


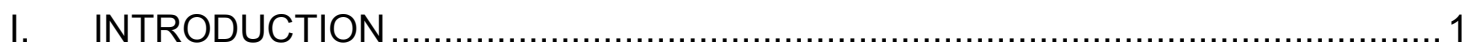

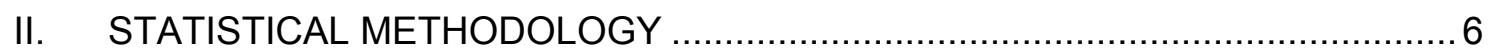

2.1 Regression models in orthogonal form and their MSEs .............................. 6

2.2 Risk functions of Estimators ………................................................. 8

2.2.1 Risk function of Ridge regression estimator ................................. 9

2.2.2 Risk function of Liu estimator.................................................... 10

III. ANALYSIS OF DOMINANCE PROPERTIES OF THE ESTIMATORS …...........12

3.1 Comparison of LASSO with Least Square Estimator .................................12

3.2 Comparison of LASSO with Ridge regression Estimator ............................ 12

3.3 Comparison of LASSO with Liu Estimator ............................................... 13

3.4 Comparison of Liu with Ridge regression Estimator.................................... 13

3.5 Comparison of Liu with Least Square Estimator ....................................... 14

3.6 Comparison of Ridge regression with Least Square Estimator .................... 14

IV. COMPARISON OF RIDGE, LIU AND TWO PARAMETER BIASED

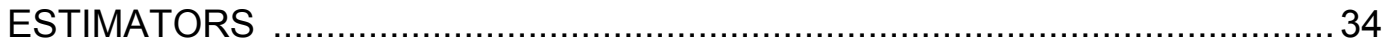

4.1 Ridge, Liu and Two parameter biased estimators .....................................34

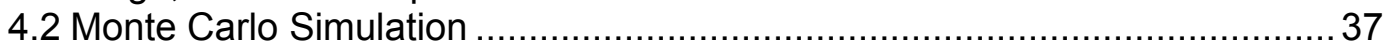

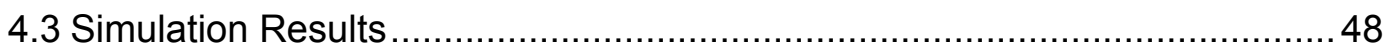

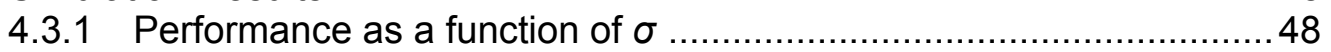

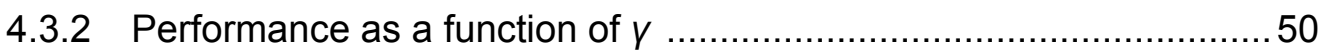

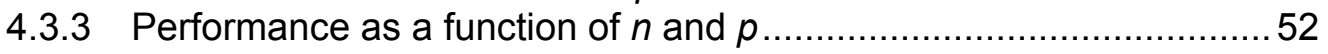

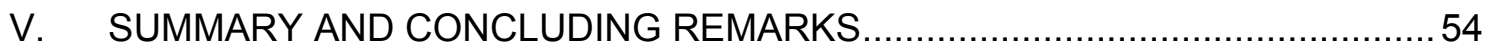

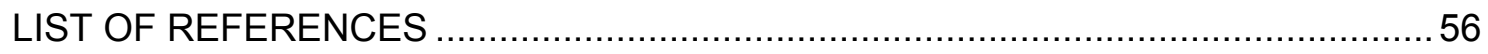




\section{INTRODUCTION}

Regression is a statistical technique for determining relationship between variables, this relationship is formulated by a statistical equation. This statistical equation allows us to predict the values of dependent variable on the basis of fixed values of one or more independent variables(or regressors or predictors), which is called regression equation or prediction model and the technique is called regression analysis. Along with the dependent variable and known independent variables, a regression equation also contains unknown regression coefficients. The main goal of a regression analysis is to appropriately estimate the values of regression coefficients and fit a good model. Regression analysis is used in almost all fields including psychology, economics, engineering, management, biology and sociology (for examples, see Mansson and Kibria (2012), Liu (2003)). Sir Francis Galton first introduced regression analysis in 1880 s in his studies of hereditary and eugenics. A regression equation with a degree of one is called linear regression equation. The simplest form of linear regression is with one dependent and only one independent variable and it is called the simple linear regression model. Usually, the dependent variable is explained by more than one variable, and we use multiple linear regression model. The standard multiple linear regression model is expressed as

$$
y=\mathbf{X} \beta+\varepsilon
$$

where $y$ is a $n \times 1$ vector of response variable, $\mathbf{X}$ is a design matrix of order $n \times p, \beta$ is a $p \times 1$ vector of regression coefficients and $\varepsilon$ is a $n \times 1$ vector of random error, which is normally

distributed with mean vector 0 and variance $\sigma^{2} \mathbf{I}_{n}$. Here $\mathbf{I}_{n}$ is identity matrix of order $\mathrm{n}$. The least square estimator (LSE) of $\beta$ is a linear function of $y$ and is defined as 


$$
\hat{\beta}=\left(\mathbf{X}^{\prime} \mathbf{X}\right)^{-1} \mathbf{X}^{\prime} y
$$

and the covariance matrix of $\hat{\beta}$ is obtained as

$$
\operatorname{cov}(\hat{\beta})=\sigma^{2}\left(\mathbf{X}^{\prime} \mathbf{X}\right)^{-1} .
$$

It is noted that the least squares estimator is unbiased and has a minimum variance. Naturally, we deal with data where the variables may or may not be independent, thus making the $\mathbf{X}^{\prime} \mathbf{X}$ matrix ill-conditioned (that is, near linear dependency among various columns of $\mathbf{X}^{\prime} \mathbf{X}$ ). We see from equations (1.2) and (1.3) that the LSE and its variancecovariance matrix heavily depend on the property of $\mathbf{X}^{\prime} \mathbf{X}$ matrix. The dependence of the columns of $\mathbf{X}$ matrix leads to the problem of multicollinearity and produce a number of errors in estimating $\beta$ which affects the reliability of the statistical inference.

To overcome this multicollinearity problem, Hoerl and Kennard (1970) introduced a new kind of estimator, the ridge regression estimator, where they proposed to add a small positive number to the diagonal elements of the $\mathbf{X}^{\prime} \mathbf{X}$ matrix. The ridge regression estimator proposed by Hoerl and Kennard is given by

$$
\hat{\beta}_{k}=\left(\mathbf{X}^{\prime} \mathbf{X}+k \mathbf{I}_{\boldsymbol{p}}\right)^{-1} \mathbf{X}^{\prime} y, \quad k \geq 0 .
$$

For a small positive value of $k$, this estimator provides a smaller mean squared error (MSE) compared to the LSE. The constant $k$ is called the ridge or biased parameter. Literature reveals a lot of discussion related to estimating a good estimator of $k$, which is to be estimated from the real data. The estimation of $k$ are discussed by Hoerl and Kennard (1970), Golub et al. (1979), Kibria (2003), Saleh (2006), Muniz and Kibria (2009), Dorugade (2013), Aslam (2014), Hefnawy and Farag (2014), and very recently Kibria and Banik (2015) among others. 
Motivated by the interpretation of the ridge estimator, Liu (1993), to combat the multicollinearity problem proposed a new class of biased estimate, the Liu estimator, defined as

$$
\hat{\beta}_{d}=\left(\mathbf{X}^{\prime} \mathbf{X}+\mathbf{I}_{\boldsymbol{p}}\right)^{-1}\left(\mathbf{X}^{\prime} y+d \hat{\beta}\right), \quad 0<d<1 .
$$

For any value of $d$, this estimator provides a smaller mean squared error compared to the least square estimator. The constant $d$ is called the shrinkage parameter. The advantage of the Liu estimator over the ridge estimator, which is a complex function of $k$, is that $\hat{\beta}_{d}$ is a linear function of $d$ and so it is convenient.

Hoerl and Kennard (1970) suggested that the appropriate range of $k$ is between 0 to 1 , but in application the chosen $k$ may not be large enough to correct the ill conditioning problem, especially when $\mathbf{X}^{\prime} \mathbf{X}$ is severely ill conditioned. In this case, the small $k$ may not be able to reduce the condition number of $\mathbf{X}^{\prime} \mathbf{X}+k \mathbf{I}_{\boldsymbol{p}}$ to proper extent, thus the resulting ridge regression may still remain unstable. This reason of instability motivated Liu (2003) to propose a new two parameter biased estimator which is defined as

$$
\hat{\beta}_{k, d}=\left(\mathbf{X}^{\prime} \mathbf{X}+k \mathbf{I}_{\boldsymbol{p}}\right)^{-1}\left(\mathbf{X}^{\prime} y-d \tilde{\beta}\right), \quad k>0,-\infty<d<\infty .
$$

where $\tilde{\beta}$ can be any estimator of $\beta . \hat{\beta}_{k, d}$ is generalization of $\hat{\beta}_{d}=\left(\mathbf{X}^{\prime} \mathbf{X}+\mathbf{I}_{\boldsymbol{p}}\right)^{-1}\left(\mathbf{X}^{\prime} y+\right.$ $d \hat{\beta})$ when $\tilde{\beta}=\hat{\beta}_{L S}$, which is the Liu estimator.

When $\tilde{\beta}=\hat{\beta}_{R}, \hat{\beta}_{k, d}=\left(\mathbf{X}^{\prime} \mathbf{X}+k \mathbf{I}_{\boldsymbol{p}}\right)^{-1} \mathbf{X}^{\prime} y-d\left(\mathbf{X}^{\prime} \mathbf{X}+k \mathbf{I}_{\boldsymbol{p}}\right)^{-2} \mathbf{X}^{\prime} y$, the estimator can fully address the ill conditioning problem. For any $k>0$, we can always find a value of $d$ so that the mean squared error provided by this estimator is less than or equal to that provided by ridge estimator. 
I will briefly discuss about the above three estimators in the latter part of the thesis, where I compare them under multicollinear regression model and error assuming a normal distribution. The comparison will be made using optimum value of $d$ proposed by Liu (2003) and few suggested ks from the literature.

The least square estimator, ridge regression estimator and Liu estimator were not considered satisfactory because, least square estimates have large variance hence less prediction accuracy. Also, with large number of predictors we would like to determine smaller subsets that has the strongest effects and thus produce easily interpretable models. On the other hand ridge regression and Liu estimators are continuous process that shrink coefficients and thus are more stable; however the problem of interpreting model with large predictors still remain unsolved as they do not set any of the coefficients to 0.Tibshirani (1996) proposed a new technique, called the LASSO, for 'least absolute shrinkage and selection operator'. It minimizes the residual sum of squares subject to the sum of the absolute value of the coefficients being less than a constant. Because of this nature of the constraint it shrinks some coefficients and tends to set others to exactly 0 , thus retaining "selection" a good feature of subset selection method and "shrinking of coefficients" a good feature of ridge regression and Liu estimator. Because of these good features the LASSO gives interpretable models.

Suppose $x^{i}=\left(x_{i 1}, \ldots, x_{i p}\right)^{\prime}, i=1,2, \ldots, n$ are the predictor variables and $y_{i}$ are responses. I assume that the $x_{i j}$ are standardized so that $\sum_{i} x_{i j} / n=0, \sum_{i} x_{i j}^{2} / n=1$.

Letting $\hat{\beta}=\left(\hat{\beta}_{1}, \ldots, \hat{\beta}_{p}\right)^{\prime}$, the LASSO estimate $(\hat{\alpha}, \hat{\beta})$ is obtained as follow,

$$
(\hat{\alpha}, \hat{\beta})=\arg \min \left\{\sum_{i=1}^{n}\left(y_{i}-\alpha-\sum_{j} \beta_{j} x_{i j}\right)^{2}\right\}, \quad \text { subject to } \sum_{j}\left|\beta_{j}\right| \leq \mathrm{t} .
$$


Here $t \geq 0$ is a tuning parameter. For all $t$, the solution for $\alpha$ is $\hat{\alpha}=\bar{y}$. I assume without loss of generality that $\bar{y}=0$ and hence omit $\alpha$.

The purpose of this research is to investigate the least square estimator, ridge regression estimator, Liu estimator and LASSO estimator and make an analytical comparison amongst them. This analytical comparison will be made under orthonormal regression model and based on the smallest mean squared error or risk and efficiency over least square estimator.

The organization of the thesis is as follows: The risk functions of the proposed estimators under the orthonormal model is given in Chapter II. Chapter III contains details of analysis of risks and efficiencies of the estimators with the tables and graphs. In Chapter IV, I reviewed some estimators of $k$ and $d$ and use Monte Carlo simulation to evaluate the performance of all estimators. Finally some concluding remarks are given in Chapter V. 


\section{STATISTICAL METHODOLOGY}

To make an analytical comparison, I have expressed all risk functions under the orthogonal regression model in this chapter. It is noted that we are restricted to compare the performance of the estimators under the orthonormal regression model as the risk of LASSO is available under the orthonormal regression model.

\subsection{Regression models in orthogonal form and their MSEs}

From (1.1) we have the multiple linear regression model as,

$$
y=\mathbf{X} \beta+\varepsilon
$$

Suppose, there exists an orthogonal matrix $\mathbf{Q}$ whose columns constitute the eigen vectors of $\mathbf{X}^{\prime} \mathbf{X}$, then $\mathbf{Q}^{\prime} \mathbf{X}^{\prime} \mathbf{X} \mathbf{Q}=\mathbf{\Lambda}=\operatorname{diag}\left(\lambda_{1}, \lambda_{2}, \ldots, \lambda_{p}\right)$, where $\lambda_{1} \geq \lambda_{2} \geq \ldots . \lambda_{p}>0$ are ordered eigenvalues of $\mathbf{X}^{\prime} \mathbf{X}$. Thus the canonical form of (1.1) is

$$
y=\mathbf{X}^{*} \alpha+\varepsilon
$$

where $\mathbf{X}^{*}=\mathbf{X} \mathbf{Q}$ and $\alpha=\mathbf{Q}^{\prime} \beta$. Here the least square estimate is given as

$$
\hat{\alpha}_{l s}=\Lambda^{-1} \mathbf{Q}^{\prime} \mathbf{y}
$$

The ridge regression approach replaces $\mathbf{X}^{\prime} \mathbf{X}$ with $\mathbf{X}^{\prime} \mathbf{X}+k \mathbf{l}$, which is same as replacing $\lambda_{\mathrm{i}}$ with $\lambda_{\mathrm{i}}+k$. Then the generalized ridge regression estimators of $\alpha$ are given as

$$
\hat{\alpha}_{k}=(\mathbf{\Lambda}+\mathbf{K} \mathbf{I})^{-1} \mathbf{Q}^{\prime} \mathbf{y}
$$

where, $\mathbf{K}=\operatorname{diag}\left(k_{1}, k_{2}, \ldots, k_{\mathrm{p}}\right), k_{\mathrm{i}}>0$. The relationship between both models is as $\hat{\beta}_{k}=\mathbf{Q} \hat{\alpha}_{k}$. Now, MSE $\left(\hat{\beta}_{k}\right)=\operatorname{MSE}\left(\hat{\alpha}_{k}\right)$ MSE $\left(\hat{\alpha}_{k}\right)$ is obtained as,

$$
\operatorname{MSE}\left(\hat{\alpha}_{k}\right)=\sigma^{2} \sum_{i=1}^{p} \frac{\lambda_{i}}{\left(\lambda_{i}+k_{i}\right)^{2}}+\sum_{i=1}^{p} \frac{k_{i}^{2} \lambda_{i}^{2}}{\left(\lambda_{i}+k_{i}\right)^{2}}, \quad k>0
$$


The Liu estimator of $\alpha$ is given as

$$
\hat{\alpha}_{d}=(\boldsymbol{\Lambda}+\mathbf{I})^{-1}\left(\mathbf{Q}^{\prime} \mathrm{y}+d \hat{\alpha}\right)
$$

The relationship between the estimators under the linear regression model and orthogonal model is as follows:

$$
\hat{\beta}_{d}=\mathbf{Q} \hat{\alpha}_{d}
$$

The MSE of $\hat{\alpha}_{d}$ is obtained as,

$$
\operatorname{MSE}\left(\hat{\alpha}_{d}\right)=\sigma^{2} \sum_{i=1}^{p} \frac{\left(\lambda_{i}+d\right)^{2}}{\lambda_{i}\left(\lambda_{i}+1\right)^{2}}+(d-1)^{2} \sum_{i=1}^{p} \frac{\alpha_{i}^{2}}{\left(\lambda_{i}+1\right)^{2}}, \quad d>0
$$

Equations (2.4) and (2.6) provides the mean squared error (MSE) of the ridge estimator and Liu estimator respectively. The MSEs are combination of their corresponding variance of the estimator and the bias in the estimator. In (2.4) the first term on right side is the sum of variances of the parameters in $\hat{\beta}_{k}$ and the second term is the square of the bias in $\hat{\beta}_{k}$. Similarly in (2.6) the first term on right side is the sum of variances of the parameters in $\hat{\beta}_{d}$ and the second term is the square of the bias in $\hat{\beta}_{d}$.

For LASSO estimator, let us consider the canonical form with full least square estimate, orthogonal regressors and normal errors with known variance. Let $\mathbf{X}$ be $n x p$ design matrix with ijth entry $\mathbf{x}_{\mathrm{ij}}$ and $\mathbf{X}^{\prime} \mathbf{X}=\mathbf{I}_{p}$.

The LASSO estimator equals,

$$
\hat{\beta}^{L}=\left(t\left(\hat{\beta}_{1}\right), t\left(\hat{\beta}_{2}\right), \ldots, t\left(\hat{\beta}_{p}\right)\right)^{\prime}
$$

where $t(x)=\operatorname{sign}(x)(|x|-\lambda)_{+}$which is exactly same as soft shrinkage proposals of Donoho and Johnstone (1994). Here, $\lambda$ is the tuning parameter. 
For any estimator $\bar{\beta}$ of $\beta$, one may define the normalized mean squared error or risk as,

$$
\mathrm{R}(\bar{\beta}, \beta)=\frac{E(\bar{\beta}-\beta)^{\prime}(\bar{\beta}-\beta)}{p \sigma_{n}^{2}}
$$

Now taking the LASSO estimator, For $\lambda=\sigma_{n} \sqrt{2 \ln (p)}$, its risk satisfies the bound

$$
\mathrm{R}\left(\hat{\beta}^{L}, \beta\right) \leq \sigma^{2}(1+2 \ln (\mathrm{p}))\left(\frac{1}{p}+c_{n}^{L}(\beta)\right)
$$

where, $c_{n}^{L}(\beta)=\frac{1}{p} \sum_{j=1}^{p} \min \left(\frac{\beta_{j}^{2}}{\sigma_{n}^{2}}, 1\right)$.

For the case when some coefficients are non-zero and some are zero. In particular, suppose $q<p$ coefficients satisfy $\beta_{j}{ }^{2} \geq \sigma_{n}^{2}$ and remaining equal zero. Then

$c_{n}^{L}(\beta)=q / p$ so the bound in (2.9) is

$$
\mathrm{R}\left(\hat{\beta}^{L}, \beta\right) \leq \sigma^{2}(1+2 \ln (p))\left(\frac{1+q}{p}\right)
$$

which approaches zero as $p \rightarrow \infty$ with $q$ fixed. More details on this see Donoho and Johnstone (1994).

\subsection{Risk functions of Estimators}

Let $(\hat{\theta}-\theta)^{2}$ be the quadratic loss function or squared error loss function, then $\mathrm{E}(\hat{\theta}-\theta)^{2}$ is termed as the risk function of the estimator, which in fact is the mean square error (MSE) of estimator $\hat{\theta}$ of a parameter $\theta$. In this section I present the risk functions of ridge regression estimator and Liu estimator.

The risk function of LSE can be obtained as,

We know, $\operatorname{MSE}(\hat{\beta})=\mathrm{E}\left((\hat{\beta}-\beta)(\hat{\beta}-\beta)^{\prime}\right)=\sigma^{2}\left(\mathbf{X}^{\prime} \mathbf{X}\right)^{-1}$ from $(1.3)$

$\operatorname{Risk}(\hat{\beta})=\sigma^{2} \operatorname{tr}\left(\left(\mathbf{X}^{\prime} \mathbf{X}\right)^{-1}\right)$ 
Let $\mathbf{X}^{\prime} \mathbf{X}=\mathbf{I}_{\boldsymbol{p}}$

$\operatorname{Risk}(\hat{\beta})=\sigma^{2} \operatorname{tr}\left(\mathbf{I}_{\boldsymbol{p}}\right)$

$$
=\sigma^{2} p
$$

\subsubsection{Risk function of ridge regression estimator}

From (1.4) we have the ridge regression estimator as,

$$
\begin{aligned}
& \hat{\beta}_{k}=\left(\mathbf{X}^{\prime} \mathbf{X}+k \mathbf{I}_{\boldsymbol{p}}\right)^{-1} \mathbf{X}^{\prime} y \\
& =\left(\mathbf{I}_{\boldsymbol{p}}+k\left(\mathbf{X}^{\prime} \mathbf{X}\right)^{-1}\right)^{-1}\left(\mathbf{X}^{\prime} \mathbf{X}\right)^{-1} \mathbf{X}^{\prime} y \\
& \text { Let } \mathbf{W}=\left(\mathbf{I}_{\boldsymbol{p}}+k\left(\mathbf{X}^{\prime} \mathbf{X}\right)^{-1}\right)^{-1} \\
& \hat{\beta}_{k}=\mathbf{w} \hat{\beta} \\
& \operatorname{MSE}\left(\hat{\beta}_{k}\right)=E\left(\hat{\beta}_{k}-\beta\right)\left(\hat{\beta}_{k}-\beta\right)^{\prime} \\
& \hat{\beta}_{k}-\beta=\mathbf{W} \hat{\beta}-\mathbf{W} \beta+\mathbf{W} \beta-\beta \\
& =\mathbf{W}(\hat{\beta}-\beta)+\left(\mathbf{W}-\mathbf{I}_{\boldsymbol{p}}\right) \beta \\
& \operatorname{MSE}\left(\hat{\beta}_{k}\right)=E\left\{\left(\mathbf{W}(\hat{\beta}-\beta)+\left(\mathbf{W}-\mathbf{I}_{\boldsymbol{p}}\right) \beta\right)\left(\mathbf{W}(\hat{\beta}-\beta)+\left(\mathbf{W}-\mathbf{I}_{\boldsymbol{p}}\right) \beta\right)^{\prime}\right\} \\
& =\mathbf{W}\left(E(\hat{\beta}-\beta)(\hat{\beta}-\beta)^{\prime}\right) \mathbf{W}^{\prime}+E\left(\mathbf{W}-\mathbf{I}_{\boldsymbol{p}}\right) \boldsymbol{\beta} \boldsymbol{\beta}^{\prime}\left(\mathbf{W}-\mathbf{I}_{\boldsymbol{p}}\right)^{\prime} \\
& =\sigma^{2} \mathbf{W}\left(\mathbf{X}^{\prime} \mathbf{X}\right)^{-1} \mathbf{W}^{\prime}+\left(\mathbf{W}-\mathbf{I}_{\boldsymbol{p}}\right) \boldsymbol{\beta} \boldsymbol{\beta}^{\prime}\left(\mathbf{W}-\mathbf{I}_{\boldsymbol{p}}\right)^{\prime}
\end{aligned}
$$

$\operatorname{Risk}\left(\hat{\beta}_{k}\right)=\sigma^{2} \operatorname{tr}\left(\mathbf{W}\left(\mathbf{X}^{\prime} \mathbf{X}\right)^{-1} \mathbf{W}^{\prime}\right)+\beta^{\prime}\left(\mathbf{W}-\mathbf{I}_{\boldsymbol{p}}\right)^{2} \beta$

$$
\text { Let } \mathbf{X}^{\prime} \mathbf{X}=\mathbf{I}_{\boldsymbol{p}} \quad \Rightarrow \mathbf{W}=\left(\mathbf{I}_{\boldsymbol{p}}+k \mathbf{I}_{\boldsymbol{p}}\right)^{-1}=\frac{1}{(1+k)} \mathbf{I}_{\boldsymbol{p}}
$$




$$
\begin{aligned}
& =\sigma^{2} \operatorname{tr}\left(\frac{1}{(1+k)} \mathbf{I}_{\boldsymbol{p}} \mathbf{I}_{\boldsymbol{p}} \mathbf{I}_{\boldsymbol{p}} \frac{1}{(1+k)}\right)+\beta^{\prime}\left(\frac{1}{(1+k)} \mathbf{I}_{\boldsymbol{p}}-\mathbf{I}_{\boldsymbol{p}}\right)^{2} \beta \\
& =\frac{\sigma^{2} p}{(1+k)^{2}}+\frac{k^{2} \beta^{\prime} \beta}{(1+k)^{2}}
\end{aligned}
$$

taking, $\Delta^{2}=\beta^{\prime} \beta / \sigma^{2}$

$$
=\frac{\sigma^{2}}{(1+k)^{2}}\left[p+k^{2} \Delta^{2}\right], \quad k>0, \Delta^{2} \geq 0
$$

where, $\Delta^{2}$ is defined as the divergence parameter. It is the sum of squares of the normalized coefficients.

\subsubsection{Risk function of Liu estimator}

From (1.5) we have Liu estimator as,

$$
\begin{aligned}
& \hat{\beta}_{d}=\left(\mathbf{X}^{\prime} \mathbf{X}+\mathbf{I}_{\boldsymbol{p}}\right)^{-1}\left(\mathbf{X}^{\prime} y+d \hat{\beta}\right) \\
& =\left(\mathbf{X}^{\prime} \mathbf{X}+\mathbf{I}_{\boldsymbol{p}}\right)^{-1}\left(\mathbf{X}^{\prime} \mathbf{X}+d \mathbf{I}_{\boldsymbol{p}}\right) \hat{\beta} \\
& \text { Let } \mathbf{F}=\left(\mathbf{X}^{\prime} \mathbf{X}+\mathbf{I}_{\boldsymbol{p}}\right)^{-1}\left(\mathbf{X}^{\prime} \mathbf{X}+d \mathbf{I}_{\boldsymbol{p}}\right), \text { then } \\
& \hat{\beta}_{d}=\mathbf{F} \hat{\beta} \\
& \operatorname{MSE}\left(\hat{\beta}_{d}\right)=E\left(\hat{\beta}_{d}-\beta\right)\left(\hat{\beta}_{d}-\beta\right)^{\prime} \\
& \hat{\beta}_{d}-\beta=\mathbf{F} \hat{\beta}-\mathbf{F} \beta+\mathbf{F} \beta-\beta \\
& =\mathbf{F}(\hat{\beta}-\beta)+\left(\mathbf{F}-\mathbf{I}_{\boldsymbol{p}}\right) \beta \\
& \operatorname{MSE}\left(\hat{\beta}_{d}\right)=E\left\{\left(\mathbf{F}(\hat{\beta}-\beta)+\left(\mathbf{F}-\mathbf{I}_{\boldsymbol{p}}\right) \beta\right)\left(\mathbf{F}(\hat{\beta}-\beta)+\left(\mathbf{F}-\mathbf{I}_{\boldsymbol{p}}\right) \beta\right)^{\prime}\right\} \\
& \quad=\mathbf{F}\left(E(\hat{\beta}-\beta)(\hat{\beta}-\beta)^{\prime}\right) \mathbf{F}^{\prime}+E\left(\mathbf{F}-\mathbf{I}_{\boldsymbol{p}}\right) \boldsymbol{\beta} \boldsymbol{\beta}^{\prime}\left(\mathbf{F}-\mathbf{I}_{\boldsymbol{p}}\right)^{\prime}
\end{aligned}
$$




$$
=\sigma^{2} \mathbf{F}\left(\mathbf{X}^{\prime} \mathbf{X}\right)^{-1} \mathbf{F}^{\prime}+\left(\mathbf{F}-\mathbf{I}_{p}\right) \boldsymbol{\beta} \boldsymbol{\beta}^{\prime}\left(\mathbf{F}-\mathbf{I}_{p}\right)^{\prime}
$$

$$
\begin{aligned}
& \operatorname{Risk}\left(\hat{\beta}_{d}\right)=\sigma^{2} \operatorname{tr}\left(\mathbf{F}\left(\mathbf{X}^{\prime} \mathbf{X}\right)^{-1} \mathbf{F}^{\prime}\right)+\beta^{\prime}\left(\mathbf{F}-\mathbf{I}_{p}\right)^{2} \beta \\
& \qquad \operatorname{Let} \mathbf{X} \mathbf{X}=\mathbf{I}_{\boldsymbol{p}} \Rightarrow \mathbf{F}=\left(\mathbf{I}_{\boldsymbol{p}}+\mathbf{I}_{\boldsymbol{p}}\right)^{-1}\left(\mathbf{I}_{\boldsymbol{p}}+d \mathbf{I}_{\boldsymbol{p}}\right) \quad=\frac{(1+d)}{2} \mathbf{I}_{\boldsymbol{p}} \\
& =\sigma^{2} \operatorname{tr}\left(\frac{(1+d)}{2} \mathbf{I}_{\boldsymbol{p}} \mathbf{I}_{\boldsymbol{p}} \mathbf{I}_{\boldsymbol{p}} \frac{(1+d)}{2}\right)+\beta^{\prime}\left(\frac{(1+d)}{2} \mathbf{I}_{\boldsymbol{p}}-\mathbf{I}_{\boldsymbol{p}}\right)^{2} \beta \\
& =\frac{\sigma^{2}(1+d)^{2} p}{4}+\frac{(d-1)^{2} \beta^{\prime} \beta}{4}
\end{aligned}
$$

taking, $\Delta^{2}=\beta^{\prime} \beta / \sigma^{2}$

$$
\operatorname{Risk}\left(\hat{\beta}_{d}\right)=\frac{\sigma^{2}}{4}\left[(1+d)^{2} p+(d-1)^{2} \Delta^{2}\right], \quad d>0, \Delta^{2} \geq 0 .
$$

where, $\Delta^{2}$ is defined as the divergence parameter. 


\section{ANALYSIS OF DOMINANCE PROPERTIES OF THE ESTIMATORS}

In this chapter, I consider the risks and relative efficiencies comparison of various estimators using the risk functions from (2.10), (2.11), (2.12) and (2.13). The relative efficiencies of each estimator is compared with LSE, which is simply the ratio of risk of LSE to risk of corresponding estimator. I computed risks and provided them as tabular form in Tables 3.1-3.8 (for fixed $p$ and different values of $\Delta^{2}$ ) and graphically presented in Figures 3.1-3.8 for different $p$. The efficiencies are provided as tabular form in Tables 3.9-3.12 for $p=3,5,7$ and 10 with graphical presentation in Figures 3.9-3.12.

\subsection{Comparison of LASSO with Least Square Estimator.}

The risk of LASSO will be less than that of LSE of $\beta$ when,

$$
\begin{gathered}
\mathrm{R}\left(\hat{\beta}^{L}\right)-\mathrm{R}(\hat{\beta})<0 \\
\sigma^{2}(1+2 \ln (\mathrm{p}))\left(\frac{1+q}{p}\right)-\mathrm{p} \sigma^{2}<0 \\
q<\frac{p^{2}}{(1+2 \ln (p))}-1
\end{gathered}
$$

Thus for all $q$ satisfying (3.1), the risk of LASSO will be less than that of LSE.

\subsection{Comparison of LASSO with Ridge regression Estimator.}

For fixed $k$ and $q$, the risk of LASSO to be less than that of ridge regression estimator when,

$$
\begin{gathered}
\mathrm{R}\left(\hat{\beta}^{L}\right)-\mathrm{R}\left(\hat{\beta}_{k}\right)<0 \\
\sigma^{2}(1+2 \ln (\mathrm{p}))\left(\frac{1+q}{p}\right)-\frac{\sigma^{2}}{(1+k)^{2}}\left[p+k^{2} \Delta^{2}\right]<0 \\
\Delta^{2}>\frac{(1+2 \ln (p))(1+q)(1+k)^{2}-p^{2}}{p k^{2}} .
\end{gathered}
$$


For all $\Delta^{2}$ satisfying (3.2) risk of LASSO will be less than that of ridge regression estimator. Otherwise, ridge regression estimator will have smaller risk than that of LASSO.

\subsection{Comparison of LASSO with Liu Estimator.}

For fixed $k$ and $q$, the risk of LASSO to be less than that of Liu estimator when,

$$
\begin{gathered}
\mathrm{R}\left(\hat{\beta}^{L}\right)-\mathrm{R}\left(\hat{\beta}_{d}\right)<0 \\
\sigma^{2}(1+2 \ln (\mathrm{p}))\left(\frac{1+q}{p}\right)-\frac{\sigma^{2}}{4}\left[(1+d)^{2} p+(d-1)^{2} \Delta^{2}\right]<0 \\
\Delta^{2}>\frac{4(1+2 \ln (p))(1+q)-((1+d) p)^{2}}{(d-1)^{2}}
\end{gathered}
$$

Otherwise, Liu will dominate LASSO estimator.

\subsection{Comparison of Liu with Ridge regression Estimator.}

The risk of Liu estimator to be less than that of ridge regression estimator when,

$$
\begin{gathered}
\mathrm{R}\left(\hat{\beta}_{d}\right)-\mathrm{R}\left(\hat{\beta}_{k}\right)<0 \\
\frac{\sigma^{2}}{4}\left[(1+d)^{2} p+(d-1)^{2} \Delta^{2}\right]-\frac{\sigma^{2}}{(1+k)^{2}}\left[p+k^{2} \Delta^{2}\right]<0
\end{gathered}
$$

When $k=d$

$$
\Delta^{2}<\frac{p\left[4-(1+k)^{4}\right]}{\left[\left(k^{2}-1\right)^{2}-4 k^{2}\right]}
$$

Otherwise ridge will dominate Liu estimator. 


\subsection{Comparison of Liu with Least Square Estimator.}

The risk of Liu estimator will be less than that of the least square estimator when,

$$
\begin{gathered}
\mathrm{R}\left(\hat{\beta}_{d}\right)-\mathrm{R}(\hat{\beta})<0 \\
\frac{\sigma^{2}}{4}\left[(1+d)^{2} p+(d-1)^{2} \Delta^{2}\right]-p \sigma^{2}<0 \\
\Delta^{2}<\frac{p\left[4-(1+d)^{2}\right]}{(d-1)^{2}}
\end{gathered}
$$

For all values of $\Delta^{2}$ satisfying (3.5), Liu estimator dominates the least square estimator.

\subsection{Comparison of Ridge regression with Least Square Estimator.}

The risk of ridge regression estimator will be less than that of the least square estimator when,

$$
\begin{gathered}
\mathrm{R}\left(\hat{\beta}_{k}\right)-\mathrm{R}(\hat{\beta})<0 \\
\frac{\sigma^{2}}{(1+k)^{2}}\left[p+k^{2} \Delta^{2}\right]-p \sigma^{2}<0 \\
\Delta^{2}<\frac{p(2+k)}{k}
\end{gathered}
$$

Otherwise, LSE dominates the ridge regression estimator.

The risks and relative efficiencies of LASSO, ridge regression, Liu and LS estimators for different values of $k, d$ and $q$ and for $p=3,4,5,6,7,8,9,10$ are presented in Tables $3.1-3.12$ respectively. These tables are in support of the comparison among all the estimators. See also the Figures 3.1-3.12 in this respect. In these figures three different values of $k$ and $d(0.1,0.5$ and 0.9$)$ are considered and the risk line corresponding to the particular value of estimator is denoted by estimator followed by its value (e.g. k0.1 for 
when $k=0.1$ and $\mathrm{d} 0.5$ for when $d=0.5)$. We know $q<p$, but $q=0$ provides a model with no explanatory variables thus we do not consider the value 0 for $q$. In the figures, for a value of $q$ the risk line is denoted by la followed by the value of $q$ (e.g. la3 for when $q=$ 3 and la for LASSO). 


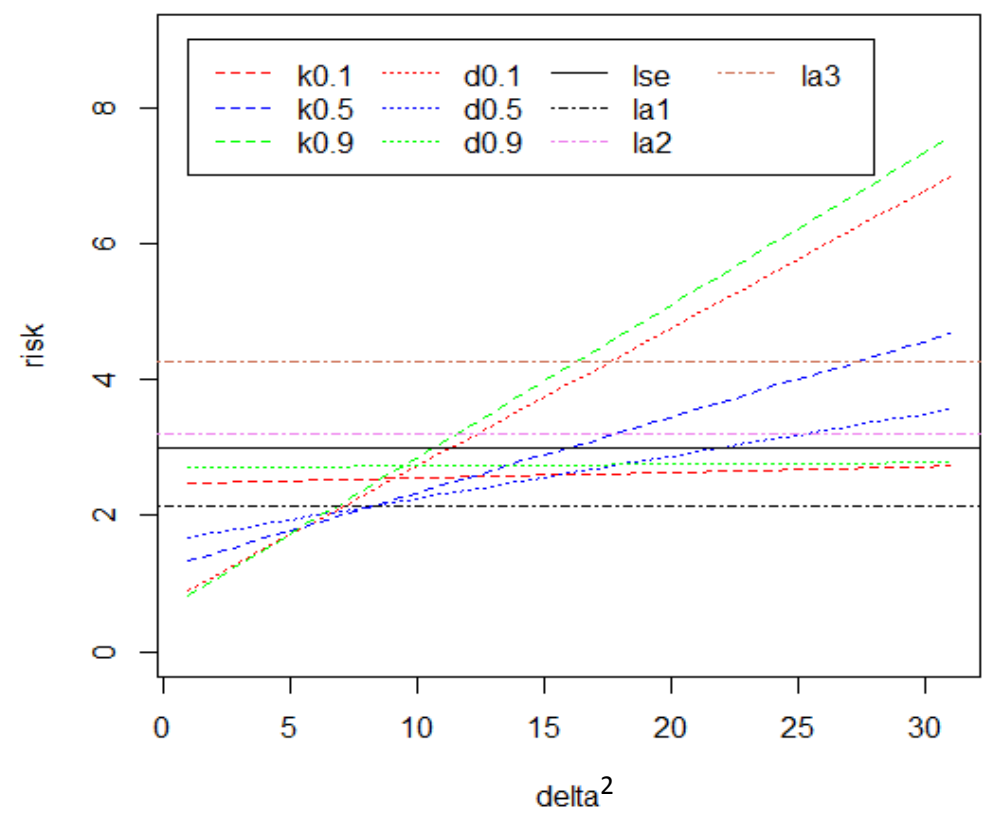

Figure 3.1: Risks of all estimators as a function of $\Delta^{2}$ for $p=3$

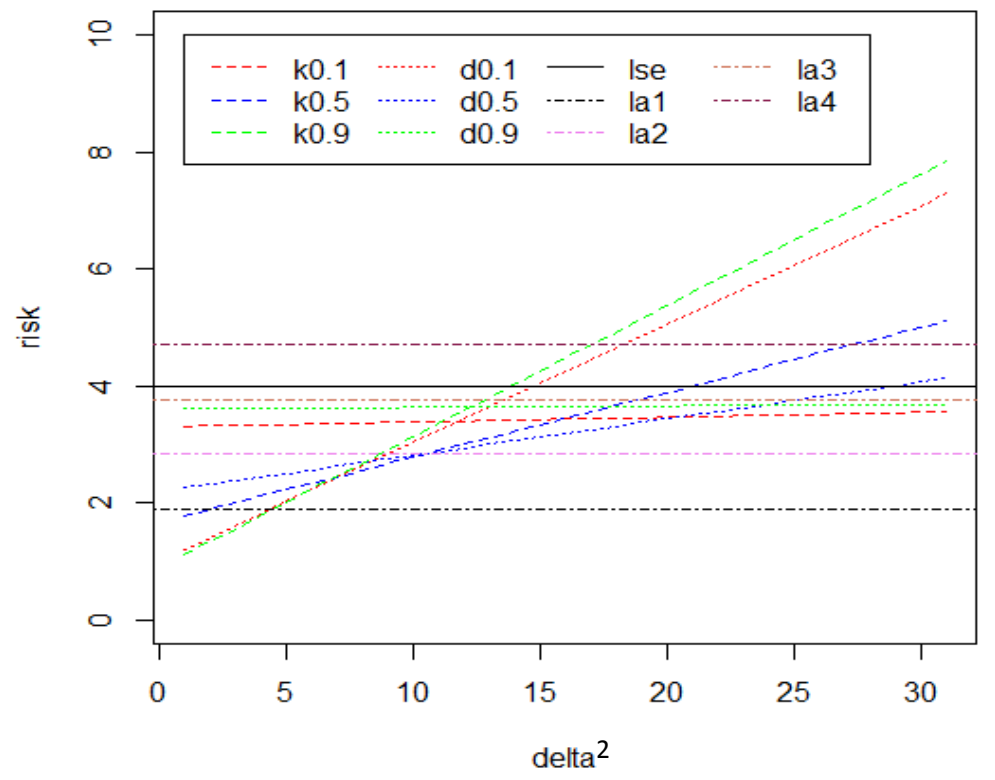

Figure 3.2: Risks of all estimators as a function of $\Delta^{2}$ for $p=4$ 


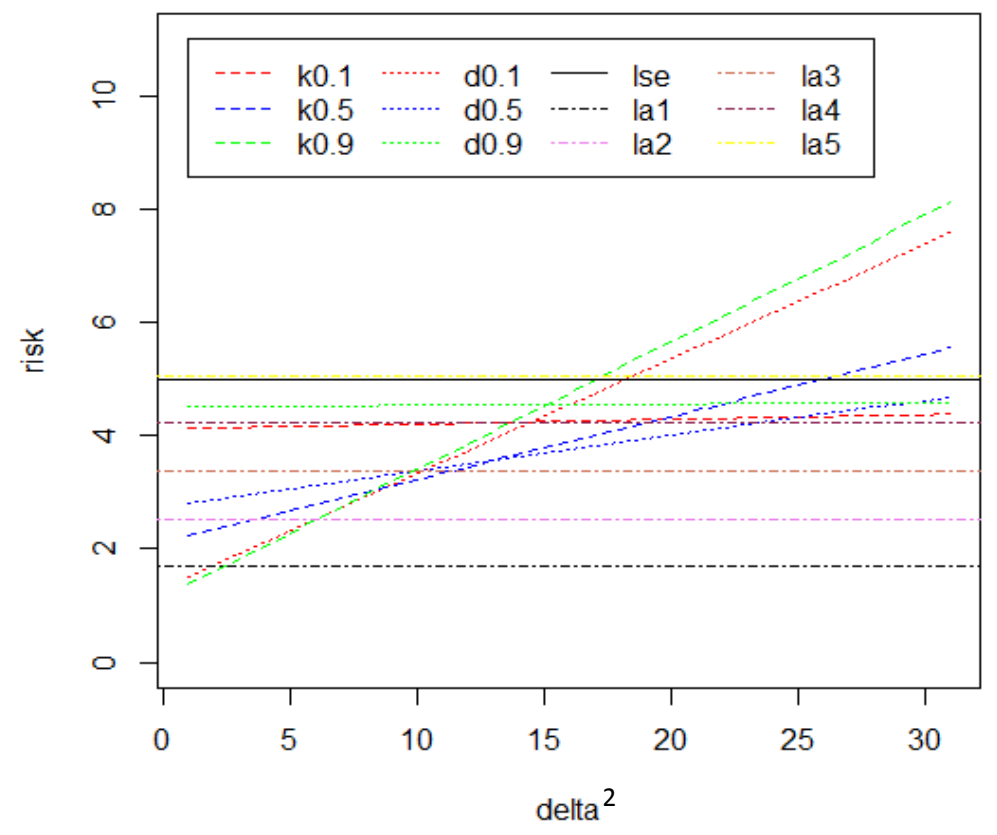

Figure 3.3: Risks of all estimators as a function of $\Delta^{2}$ for $p=5$

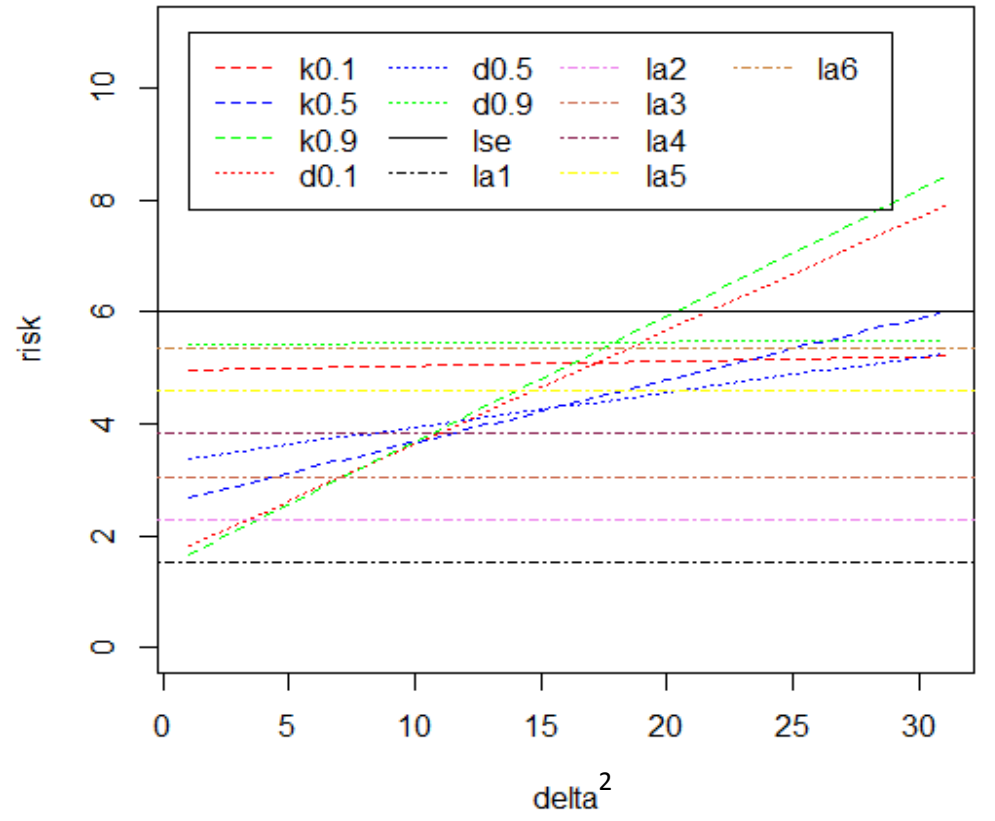

Figure 3.4: Risks of all estimators as a function of $\Delta^{2}$ for $p=6$ 


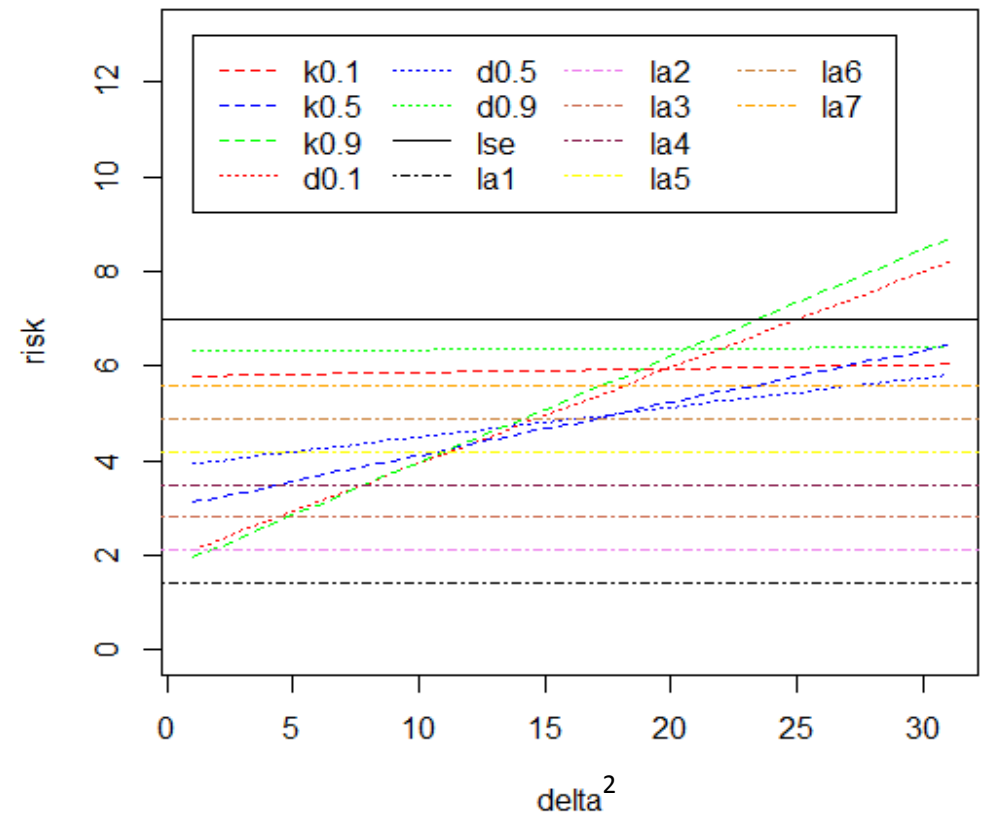

Figure 3.5: Risks of all estimators as a function of $\Delta^{2}$ for $p=7$

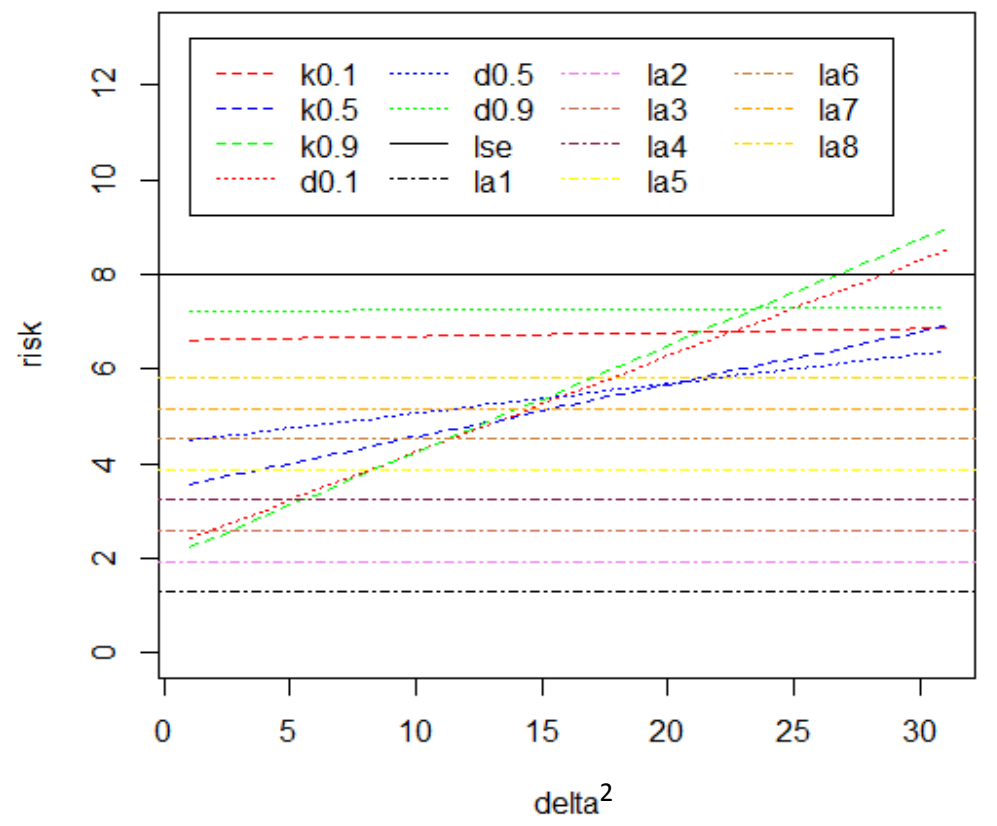

Figure 3.6: Risks of all estimators as a function of $\Delta^{2}$ for $p=8$ 


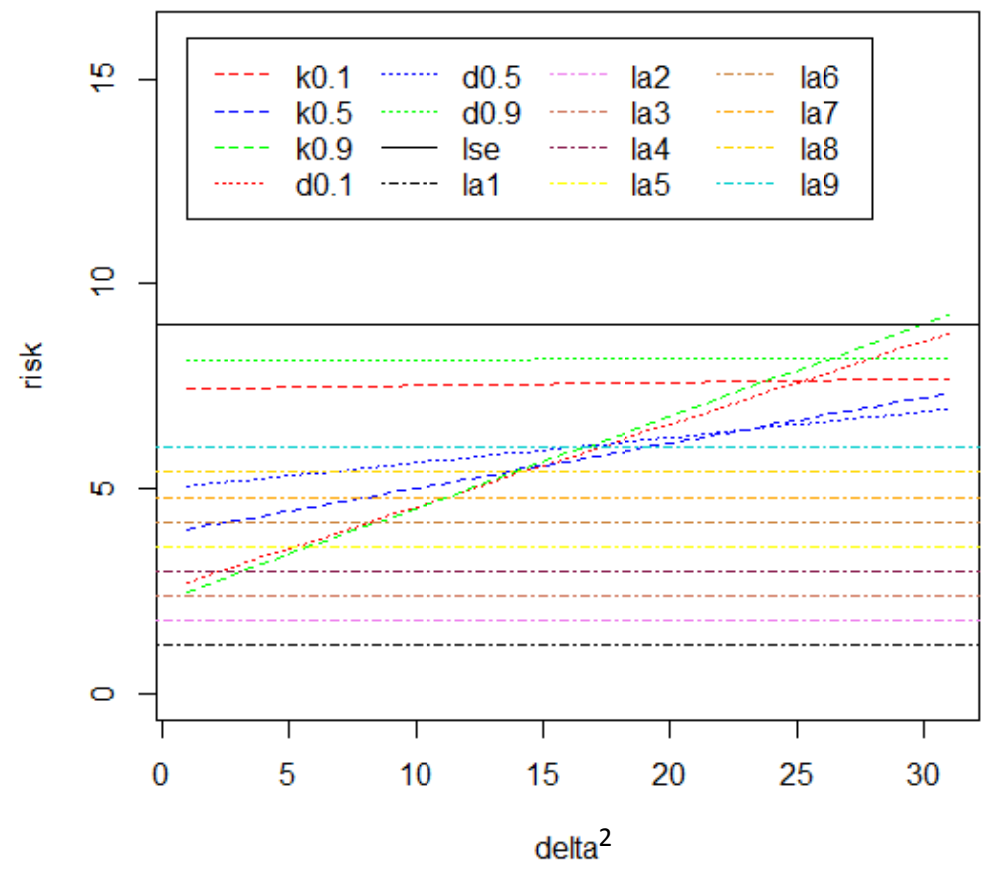

Figure 3.7: Risks of all estimators as a function of $\Delta^{2}$ for $p=9$

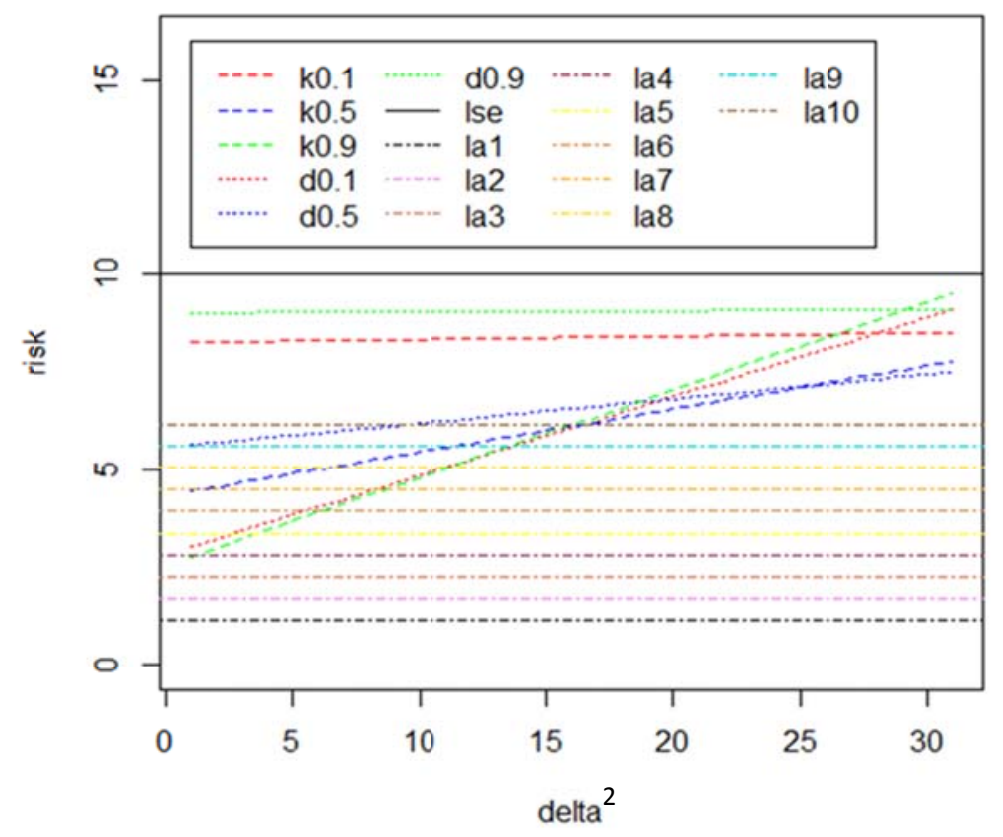

Figure 3.8: Risks of all estimators as a function of $\Delta^{2}$ for $p=10$ 


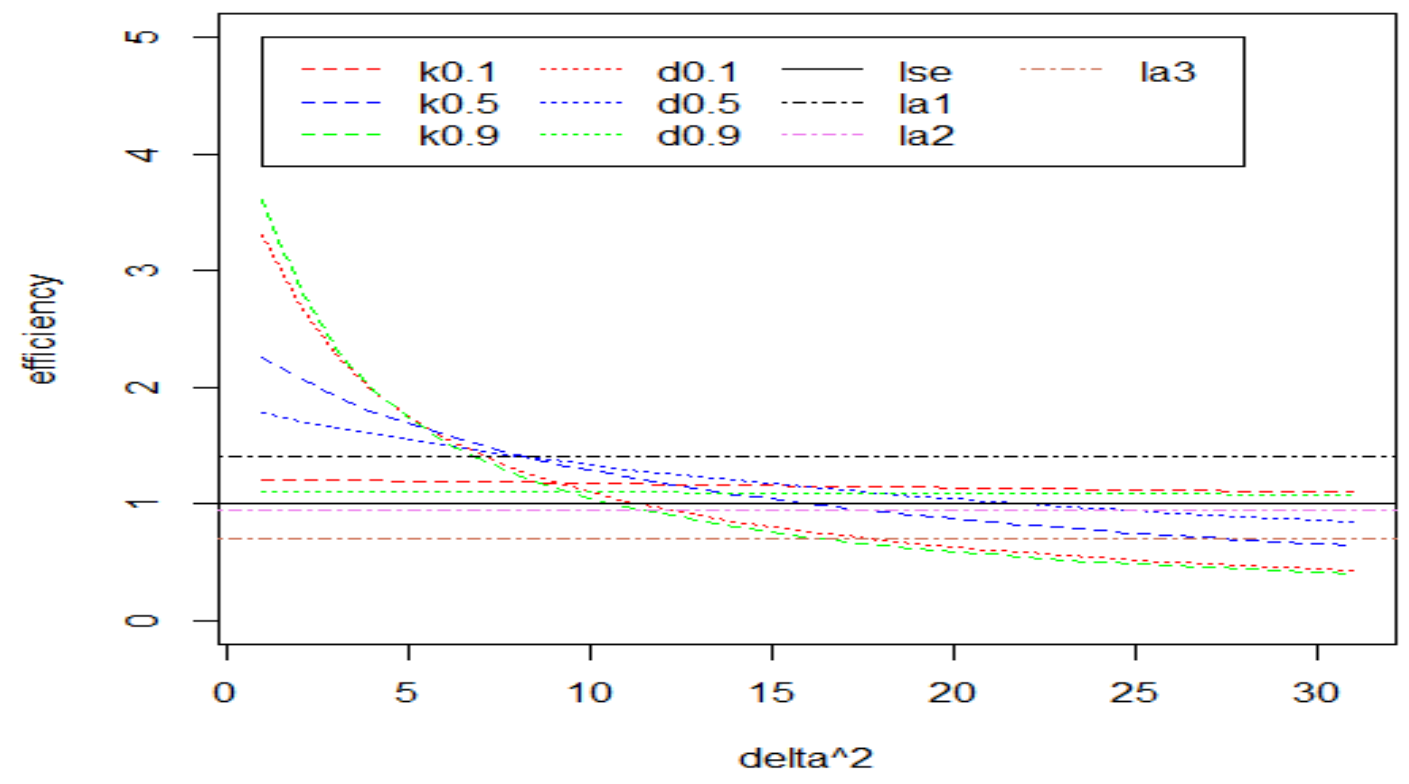

Figure 3.9: Efficiency of all estimators as a function of $\Delta^{2}$ for $p=3$

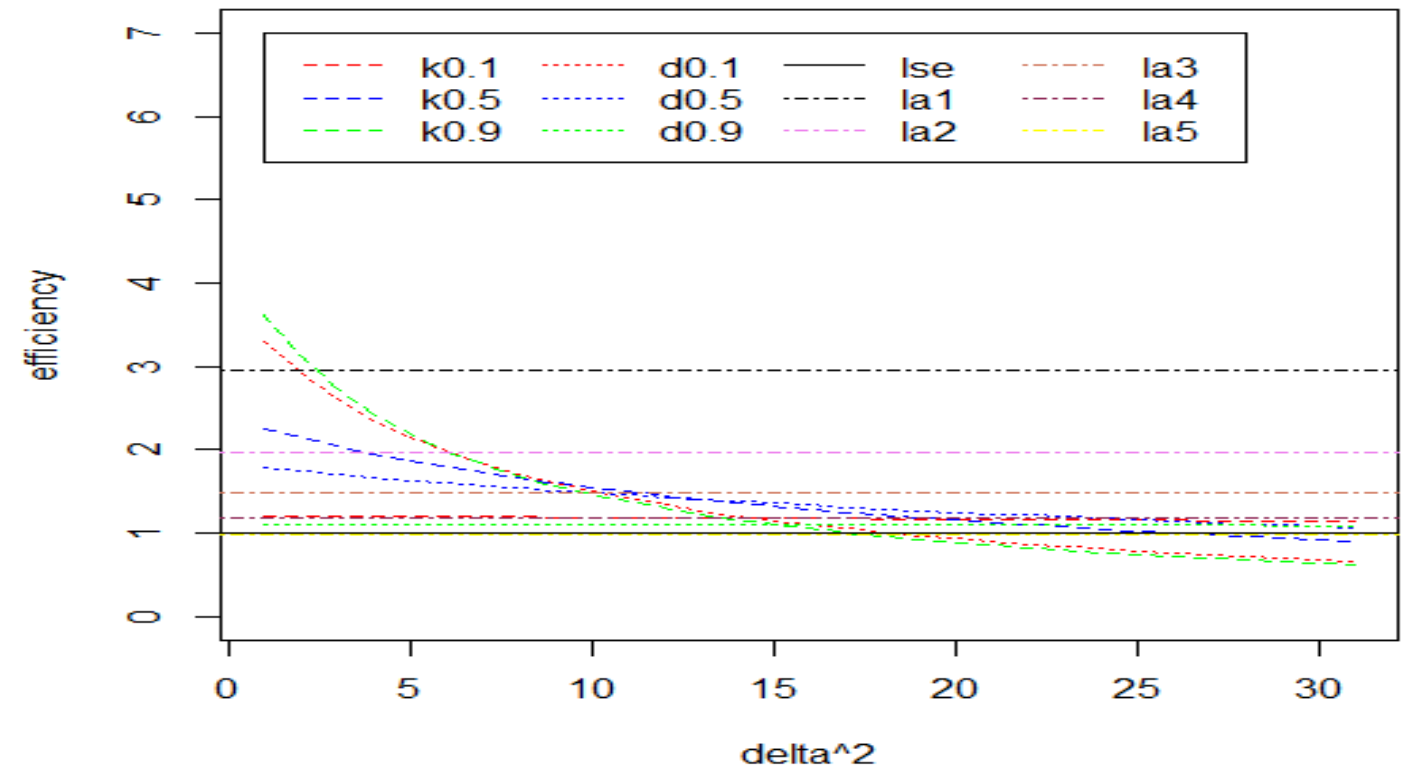

Figure 3.10: Efficiency of all estimators as a function of $\Delta^{2}$ for $p=5$ 


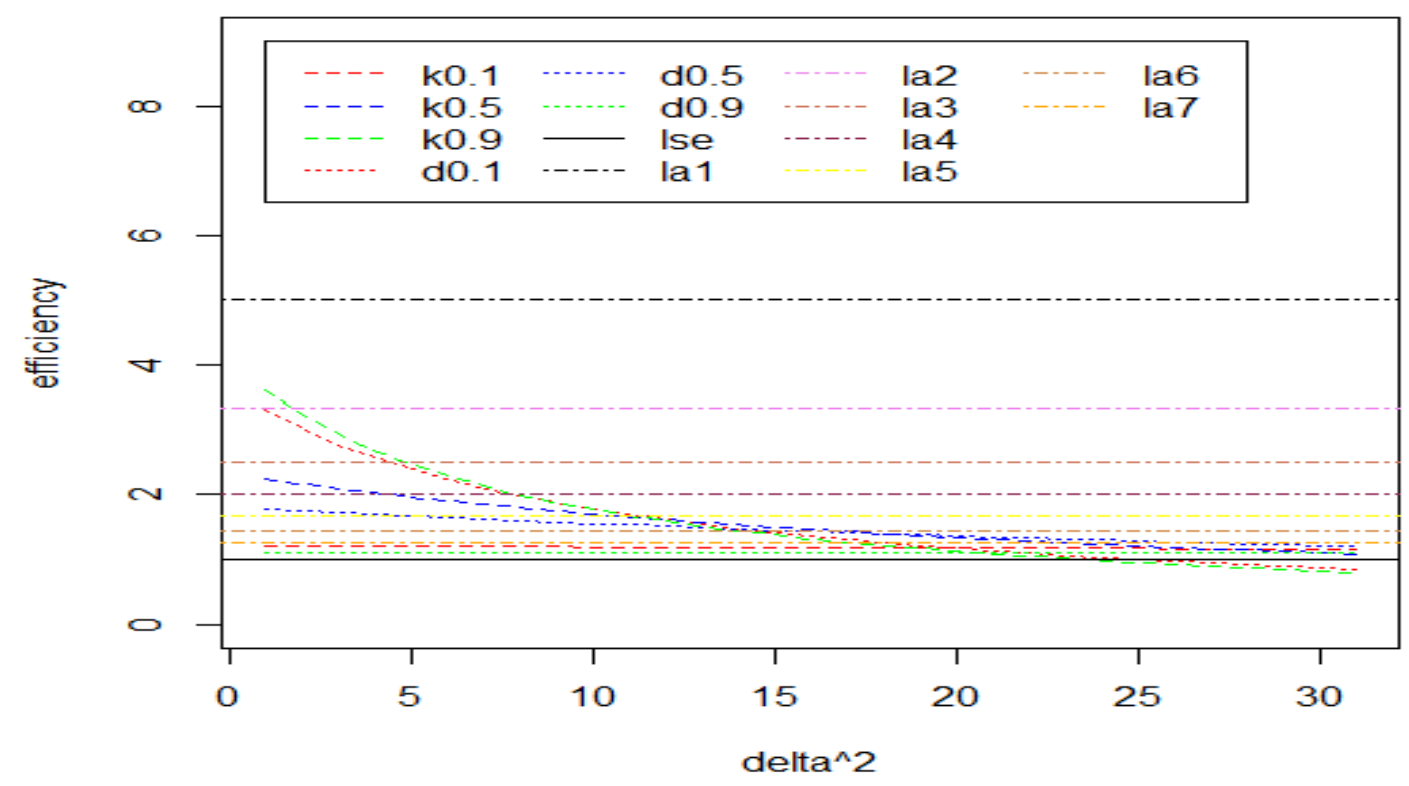

Figure 3.11: Efficiency of all estimators as a function of $\Delta^{2}$ for $p=7$

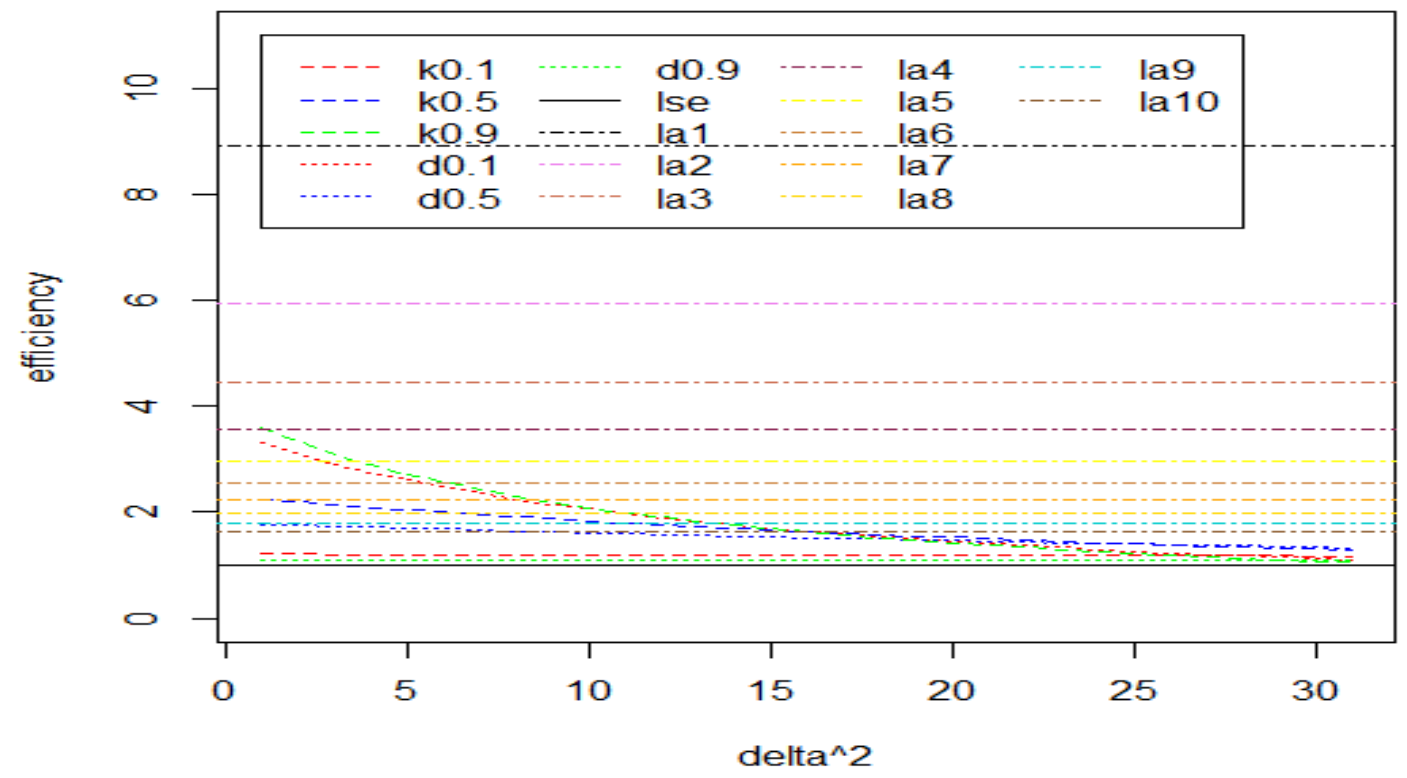

Figure 3.12: Efficiency of all estimators as a function of $\Delta^{2}$ for $p=10$ 
Table 3.1: Risk for different values of $\Delta^{2}$ at $p=3$ and $k=d=0.1,0.5$ and 0.9

\begin{tabular}{|c|c|c|c|c|c|c|c|c|c|c|}
\hline \multirow[t]{2}{*}{$\Delta^{2}$} & \multirow[t]{2}{*}{ LSE } & \multicolumn{3}{|c|}{ LASSO } & \multicolumn{3}{|c|}{ Ridge } & \multicolumn{3}{|c|}{ Liu } \\
\hline & & $q=1$ & $q=2$ & $q=3$ & $k=0.1$ & $k=0.5$ & $k=0.9$ & $d=0.1$ & $d=0.5$ & $d=0.9$ \\
\hline 0.00 & 3.00 & 2.13 & 3.20 & 4.26 & 2.48 & 1.33 & 0.83 & 0.91 & 1.69 & 2.71 \\
\hline 1.00 & 3.00 & 2.13 & 3.20 & 4.26 & 2.49 & 1.44 & 1.06 & 1.11 & 1.75 & 2.71 \\
\hline 2.00 & 3.00 & 2.13 & 3.20 & 4.26 & 2.50 & 1.56 & 1.28 & 1.31 & 1.81 & 2.71 \\
\hline 3.00 & 3.00 & 2.13 & 3.20 & 4.26 & 2.50 & 1.67 & 1.50 & 1.52 & 1.88 & 2.72 \\
\hline 4.00 & 3.00 & 2.13 & 3.20 & 4.26 & 2.51 & 1.78 & 1.73 & 1.72 & 1.94 & 2.72 \\
\hline 5.00 & 3.00 & 2.13 & 3.20 & 4.26 & 2.52 & 1.89 & 1.95 & 1.92 & 2.00 & 2.72 \\
\hline 6.00 & 3.00 & 2.13 & 3.20 & 4.26 & 2.53 & 2.00 & 2.18 & 2.12 & 2.06 & 2.72 \\
\hline 7.00 & 3.00 & 2.13 & 3.20 & 4.26 & 2.54 & 2.11 & 2.40 & 2.33 & 2.13 & 2.73 \\
\hline 8.00 & 3.00 & 2.13 & 3.20 & 4.26 & 2.55 & 2.22 & 2.63 & 2.53 & 2.19 & 2.73 \\
\hline 9.00 & 3.00 & 2.13 & 3.20 & 4.26 & 2.55 & 2.33 & 2.85 & 2.73 & 2.25 & 2.73 \\
\hline 10.00 & 3.00 & 2.13 & 3.20 & 4.26 & 2.56 & 2.44 & 3.07 & 2.93 & 2.31 & 2.73 \\
\hline 11.00 & 3.00 & 2.13 & 3.20 & 4.26 & 2.57 & 2.56 & 3.30 & 3.14 & 2.38 & 2.74 \\
\hline 12.00 & 3.00 & 2.13 & 3.20 & 4.26 & 2.58 & 2.67 & 3.52 & 3.34 & 2.44 & 2.74 \\
\hline 13.00 & 3.00 & 2.13 & 3.20 & 4.26 & 2.59 & 2.78 & 3.75 & 3.54 & 2.50 & 2.74 \\
\hline 14.00 & 3.00 & 2.13 & 3.20 & 4.26 & 2.60 & 2.89 & 3.97 & 3.74 & 2.56 & 2.74 \\
\hline 15.00 & 3.00 & 2.13 & 3.20 & 4.26 & 2.60 & 3.00 & 4.20 & 3.95 & 2.63 & 2.75 \\
\hline 16.00 & 3.00 & 2.13 & 3.20 & 4.26 & 2.61 & 3.11 & 4.42 & 4.15 & 2.69 & 2.75 \\
\hline 17.00 & 3.00 & 2.13 & 3.20 & 4.26 & 2.62 & 3.22 & 4.65 & 4.35 & 2.75 & 2.75 \\
\hline 18.00 & 3.00 & 2.13 & 3.20 & 4.26 & 2.63 & 3.33 & 4.87 & 4.55 & 2.81 & 2.75 \\
\hline 19.00 & 3.00 & 2.13 & 3.20 & 4.26 & 2.64 & 3.44 & 5.09 & 4.76 & 2.88 & 2.76 \\
\hline 20.00 & 3.00 & 2.13 & 3.20 & 4.26 & 2.64 & 3.56 & 5.32 & 4.96 & 2.94 & 2.76 \\
\hline 21.00 & 3.00 & 2.13 & 3.20 & 4.26 & 2.65 & 3.67 & 5.54 & 5.16 & 3.00 & 2.76 \\
\hline 22.00 & 3.00 & 2.13 & 3.20 & 4.26 & 2.66 & 3.78 & 5.77 & 5.36 & 3.06 & 2.76 \\
\hline 23.00 & 3.00 & 2.13 & 3.20 & 4.26 & 2.67 & 3.89 & 5.99 & 5.57 & 3.13 & 2.77 \\
\hline 24.00 & 3.00 & 2.13 & 3.20 & 4.26 & 2.68 & 4.00 & 6.22 & 5.77 & 3.19 & 2.77 \\
\hline 25.00 & 3.00 & 2.13 & 3.20 & 4.26 & 2.69 & 4.11 & 6.44 & 5.97 & 3.25 & 2.77 \\
\hline 26.00 & 3.00 & 2.13 & 3.20 & 4.26 & 2.69 & 4.22 & 6.66 & 6.17 & 3.31 & 2.77 \\
\hline 27.00 & 3.00 & 2.13 & 3.20 & 4.26 & 2.70 & 4.33 & 6.89 & 6.38 & 3.38 & 2.78 \\
\hline 28.00 & 3.00 & 2.13 & 3.20 & 4.26 & 2.71 & 4.44 & 7.11 & 6.58 & 3.44 & 2.78 \\
\hline 29.00 & 3.00 & 2.13 & 3.20 & 4.26 & 2.72 & 4.56 & 7.34 & 6.78 & 3.50 & 2.78 \\
\hline 30.00 & 3.00 & 2.13 & 3.20 & 4.26 & 2.73 & 4.67 & 7.56 & 6.98 & 3.56 & 2.78 \\
\hline
\end{tabular}


Table 3.2: Risk for different values of $\Delta^{2}$ at $p=4$ and $k=d=0.1,0.5$ and 0.9

\begin{tabular}{|c|c|c|c|c|c|c|c|c|c|c|}
\hline \multirow[t]{2}{*}{$\Delta^{2}$} & \multirow[t]{2}{*}{ LSE } & \multicolumn{3}{|c|}{ LASSO } & \multicolumn{3}{|c|}{ Ridge } & \multicolumn{3}{|c|}{ Liu } \\
\hline & & $q=1$ & $q=2$ & $q=4$ & $k=0.1$ & $k=0.5$ & $k=0.9$ & $d=0.1$ & $d=0.5$ & $d=0.9$ \\
\hline 0.00 & 4.00 & 1.89 & 3.77 & 4.72 & 3.31 & 1.78 & 1.11 & 1.21 & 2.25 & 3.61 \\
\hline 1.00 & 4.00 & 1.89 & 3.77 & 4.72 & 3.31 & 1.89 & 1.33 & 1.41 & 2.31 & 3.61 \\
\hline 2.00 & 4.00 & 1.89 & 3.77 & 4.72 & 3.32 & 2.00 & 1.56 & 1.62 & 2.38 & 3.62 \\
\hline 3.00 & 4.00 & 1.89 & 3.77 & 4.72 & 3.33 & 2.11 & 1.78 & 1.82 & 2.44 & 3.62 \\
\hline 4.00 & 4.00 & 1.89 & 3.77 & 4.72 & 3.34 & 2.22 & 2.01 & 2.02 & 2.50 & 3.62 \\
\hline 5.00 & 4.00 & 1.89 & 3.77 & 4.72 & 3.35 & 2.33 & 2.23 & 2.22 & 2.56 & 3.62 \\
\hline 6.00 & 4.00 & 1.89 & 3.77 & 4.72 & 3.36 & 2.44 & 2.45 & 2.43 & 2.63 & 3.63 \\
\hline 7.00 & 4.00 & 1.89 & 3.77 & 4.72 & 3.36 & 2.56 & 2.68 & 2.63 & 2.69 & 3.63 \\
\hline 8.00 & 4.00 & 1.89 & 3.77 & 4.72 & 3.37 & 2.67 & 2.90 & 2.83 & 2.75 & 3.63 \\
\hline 9.00 & 4.00 & 1.89 & 3.77 & 4.72 & 3.38 & 2.78 & 3.13 & 3.03 & 2.81 & 3.63 \\
\hline 10.00 & 4.00 & 1.89 & 3.77 & 4.72 & 3.39 & 2.89 & 3.35 & 3.24 & 2.88 & 3.64 \\
\hline 11.00 & 4.00 & 1.89 & 3.77 & 4.72 & 3.40 & 3.00 & 3.58 & 3.44 & 2.94 & 3.64 \\
\hline 12.00 & 4.00 & 1.89 & 3.77 & 4.72 & 3.40 & 3.11 & 3.80 & 3.64 & 3.00 & 3.64 \\
\hline 13.00 & 4.00 & 1.89 & 3.77 & 4.72 & 3.41 & 3.22 & 4.02 & 3.84 & 3.06 & 3.64 \\
\hline 14.00 & 4.00 & 1.89 & 3.77 & 4.72 & 3.42 & 3.33 & 4.25 & 4.05 & 3.13 & 3.65 \\
\hline 15.00 & 4.00 & 1.89 & 3.77 & 4.72 & 3.43 & 3.44 & 4.47 & 4.25 & 3.19 & 3.65 \\
\hline 16.00 & 4.00 & 1.89 & 3.77 & 4.72 & 3.44 & 3.56 & 4.70 & 4.45 & 3.25 & 3.65 \\
\hline 17.00 & 4.00 & 1.89 & 3.77 & 4.72 & 3.45 & 3.67 & 4.92 & 4.65 & 3.31 & 3.65 \\
\hline 18.00 & 4.00 & 1.89 & 3.77 & 4.72 & 3.45 & 3.78 & 5.15 & 4.86 & 3.38 & 3.66 \\
\hline 19.00 & 4.00 & 1.89 & 3.77 & 4.72 & 3.46 & 3.89 & 5.37 & 5.06 & 3.44 & 3.66 \\
\hline 20.00 & 4.00 & 1.89 & 3.77 & 4.72 & 3.47 & 4.00 & 5.60 & 5.26 & 3.50 & 3.66 \\
\hline 21.00 & 4.00 & 1.89 & 3.77 & 4.72 & 3.48 & 4.11 & 5.82 & 5.46 & 3.56 & 3.66 \\
\hline 22.00 & 4.00 & 1.89 & 3.77 & 4.72 & 3.49 & 4.22 & 6.04 & 5.67 & 3.63 & 3.67 \\
\hline 23.00 & 4.00 & 1.89 & 3.77 & 4.72 & 3.50 & 4.33 & 6.27 & 5.87 & 3.69 & 3.67 \\
\hline 24.00 & 4.00 & 1.89 & 3.77 & 4.72 & 3.50 & 4.44 & 6.49 & 6.07 & 3.75 & 3.67 \\
\hline 25.00 & 4.00 & 1.89 & 3.77 & 4.72 & 3.51 & 4.56 & 6.72 & 6.27 & 3.81 & 3.67 \\
\hline 26.00 & 4.00 & 1.89 & 3.77 & 4.72 & 3.52 & 4.67 & 6.94 & 6.48 & 3.88 & 3.68 \\
\hline 27.00 & 4.00 & 1.89 & 3.77 & 4.72 & 3.53 & 4.78 & 7.17 & 6.68 & 3.94 & 3.68 \\
\hline 28.00 & 4.00 & 1.89 & 3.77 & 4.72 & 3.54 & 4.89 & 7.39 & 6.88 & 4.00 & 3.68 \\
\hline 29.00 & 4.00 & 1.89 & 3.77 & 4.72 & 3.55 & 5.00 & 7.61 & 7.08 & 4.06 & 3.68 \\
\hline 30.00 & 4.00 & 1.89 & 3.77 & 4.72 & 3.55 & 5.11 & 7.84 & 7.29 & 4.13 & 3.69 \\
\hline
\end{tabular}


Table 3.3: Risk for different values of $\Delta^{2}$ at $p=5$ and $k=d=0.1,0.5$ and 0.9

\begin{tabular}{|c|c|c|c|c|c|c|c|c|c|c|}
\hline \multirow[t]{2}{*}{$\Delta^{2}$} & \multirow[t]{2}{*}{ LSE } & \multicolumn{3}{|c|}{ LASSO } & \multicolumn{3}{|c|}{ Ridge } & \multicolumn{3}{|c|}{ Liu } \\
\hline & & $q=1$ & $q=3$ & $q=5$ & $k=0.1$ & $k=0.5$ & $k=0.9$ & $d=0.1$ & $d=0.5$ & $d=0.9$ \\
\hline 0.00 & 5.00 & 1.69 & 3.38 & 5.06 & 4.13 & 2.22 & 1.39 & 1.51 & 2.81 & 4.51 \\
\hline 1.00 & 5.00 & 1.69 & 3.38 & 5.06 & 4.14 & 2.33 & 1.61 & 1.72 & 2.88 & 4.52 \\
\hline 2.00 & 5.00 & 1.69 & 3.38 & 5.06 & 4.15 & 2.44 & 1.83 & 1.92 & 2.94 & 4.52 \\
\hline 3.00 & 5.00 & 1.69 & 3.38 & 5.06 & 4.16 & 2.56 & 2.06 & 2.12 & 3.00 & 4.52 \\
\hline 4.00 & 5.00 & 1.69 & 3.38 & 5.06 & 4.17 & 2.67 & 2.28 & 2.32 & 3.06 & 4.52 \\
\hline 5.00 & 5.00 & 1.69 & 3.38 & 5.06 & 4.17 & 2.78 & 2.51 & 2.53 & 3.13 & 4.53 \\
\hline 6.00 & 5.00 & 1.69 & 3.38 & 5.06 & 4.18 & 2.89 & 2.73 & 2.73 & 3.19 & 4.53 \\
\hline 7.00 & 5.00 & 1.69 & 3.38 & 5.06 & 4.19 & 3.00 & 2.96 & 2.93 & 3.25 & 4.53 \\
\hline 8.00 & 5.00 & 1.69 & 3.38 & 5.06 & 4.20 & 3.11 & 3.18 & 3.13 & 3.31 & 4.53 \\
\hline 9.00 & 5.00 & 1.69 & 3.38 & 5.06 & 4.21 & 3.22 & 3.40 & 3.34 & 3.38 & 4.54 \\
\hline 10.00 & 5.00 & 1.69 & 3.38 & 5.06 & 4.21 & 3.33 & 3.63 & 3.54 & 3.44 & 4.54 \\
\hline 11.00 & 5.00 & 1.69 & 3.38 & 5.06 & 4.22 & 3.44 & 3.85 & 3.74 & 3.50 & 4.54 \\
\hline 12.00 & 5.00 & 1.69 & 3.38 & 5.06 & 4.23 & 3.56 & 4.08 & 3.94 & 3.56 & 4.54 \\
\hline 13.00 & 5.00 & 1.69 & 3.38 & 5.06 & 4.24 & 3.67 & 4.30 & 4.15 & 3.63 & 4.55 \\
\hline 14.00 & 5.00 & 1.69 & 3.38 & 5.06 & 4.25 & 3.78 & 4.53 & 4.35 & 3.69 & 4.55 \\
\hline 15.00 & 5.00 & 1.69 & 3.38 & 5.06 & 4.26 & 3.89 & 4.75 & 4.55 & 3.75 & 4.55 \\
\hline 16.00 & 5.00 & 1.69 & 3.38 & 5.06 & 4.26 & 4.00 & 4.98 & 4.75 & 3.81 & 4.55 \\
\hline 17.00 & 5.00 & 1.69 & 3.38 & 5.06 & 4.27 & 4.11 & 5.20 & 4.96 & 3.88 & 4.56 \\
\hline 18.00 & 5.00 & 1.69 & 3.38 & 5.06 & 4.28 & 4.22 & 5.42 & 5.16 & 3.94 & 4.56 \\
\hline 19.00 & 5.00 & 1.69 & 3.38 & 5.06 & 4.29 & 4.33 & 5.65 & 5.36 & 4.00 & 4.56 \\
\hline 20.00 & 5.00 & 1.69 & 3.38 & 5.06 & 4.30 & 4.44 & 5.87 & 5.56 & 4.06 & 4.56 \\
\hline 21.00 & 5.00 & 1.69 & 3.38 & 5.06 & 4.31 & 4.56 & 6.10 & 5.77 & 4.13 & 4.57 \\
\hline 22.00 & 5.00 & 1.69 & 3.38 & 5.06 & 4.31 & 4.67 & 6.32 & 5.97 & 4.19 & 4.57 \\
\hline 23.00 & 5.00 & 1.69 & 3.38 & 5.06 & 4.32 & 4.78 & 6.55 & 6.17 & 4.25 & 4.57 \\
\hline 24.00 & 5.00 & 1.69 & 3.38 & 5.06 & 4.33 & 4.89 & 6.77 & 6.37 & 4.31 & 4.57 \\
\hline 25.00 & 5.00 & 1.69 & 3.38 & 5.06 & 4.34 & 5.00 & 6.99 & 6.58 & 4.38 & 4.58 \\
\hline 26.00 & 5.00 & 1.69 & 3.38 & 5.06 & 4.35 & 5.11 & 7.22 & 6.78 & 4.44 & 4.58 \\
\hline 27.00 & 5.00 & 1.69 & 3.38 & 5.06 & 4.36 & 5.22 & 7.44 & 6.98 & 4.50 & 4.58 \\
\hline 28.00 & 5.00 & 1.69 & 3.38 & 5.06 & 4.36 & 5.33 & 7.67 & 7.18 & 4.56 & 4.58 \\
\hline 29.00 & 5.00 & 1.69 & 3.38 & 5.06 & 4.37 & 5.44 & 7.89 & 7.39 & 4.63 & 4.59 \\
\hline 30.00 & 5.00 & 1.69 & 3.38 & 5.06 & 4.38 & 5.56 & 8.12 & 7.59 & 4.69 & 4.59 \\
\hline
\end{tabular}


Table 3.4: Risk for different values of $\Delta^{2}$ at $p=7$ and $k=d=0.1,0.5$ and 0.9

\begin{tabular}{|c|c|c|c|c|c|c|c|c|c|c|c|}
\hline \multirow[t]{2}{*}{$\Delta^{2}$} & \multirow[t]{2}{*}{ LSE } & \multicolumn{4}{|c|}{ LASSO } & \multicolumn{3}{|c|}{ Ridge } & \multicolumn{3}{|c|}{ Liu } \\
\hline & & $q=1$ & $q=3$ & $q=5$ & $q=7$ & $k=0.1$ & $k=0.5$ & $k=0.9$ & $d=0.1$ & $d=0.5$ & $d=0.9$ \\
\hline 0.00 & 7.00 & 1.40 & 2.80 & 4.19 & 5.59 & 5.79 & 3.11 & 1.94 & 2.12 & 3.94 & 6.32 \\
\hline 1.00 & 7.00 & 1.40 & 2.80 & 4.19 & 5.59 & 5.79 & 3.22 & 2.16 & 2.32 & 4.00 & 6.32 \\
\hline 2.00 & 7.00 & 1.40 & 2.80 & 4.19 & 5.59 & 5.80 & 3.33 & 2.39 & 2.52 & 4.06 & 6.32 \\
\hline 3.00 & 7.00 & 1.40 & 2.80 & 4.19 & 5.59 & 5.81 & 3.44 & 2.61 & 2.73 & 4.13 & 6.33 \\
\hline 4.00 & 7.00 & 1.40 & 2.80 & 4.19 & 5.59 & 5.82 & 3.56 & 2.84 & 2.93 & 4.19 & 6.33 \\
\hline 5.00 & 7.00 & 1.40 & 2.80 & 4.19 & 5.59 & 5.83 & 3.67 & 3.06 & 3.13 & 4.25 & 6.33 \\
\hline 6.00 & 7.00 & 1.40 & 2.80 & 4.19 & 5.59 & 5.83 & 3.78 & 3.29 & 3.33 & 4.31 & 6.33 \\
\hline 7.00 & 7.00 & 1.40 & 2.80 & 4.19 & 5.59 & 5.84 & 3.89 & 3.51 & 3.54 & 4.38 & 6.34 \\
\hline 8.00 & 7.00 & 1.40 & 2.80 & 4.19 & 5.59 & 5.85 & 4.00 & 3.73 & 3.74 & 4.44 & 6.34 \\
\hline 9.00 & 7.00 & 1.40 & 2.80 & 4.19 & 5.59 & 5.86 & 4.11 & 3.96 & 3.94 & 4.50 & 6.34 \\
\hline 10.00 & 7.00 & 1.40 & 2.80 & 4.19 & 5.59 & 5.87 & 4.22 & 4.18 & 4.14 & 4.56 & 6.34 \\
\hline 11.00 & 7.00 & 1.40 & 2.80 & 4.19 & 5.59 & 5.88 & 4.33 & 4.41 & 4.35 & 4.63 & 6.35 \\
\hline 12.00 & 7.00 & 1.40 & 2.80 & 4.19 & 5.59 & 5.88 & 4.44 & 4.63 & 4.55 & 4.69 & 6.35 \\
\hline 13.00 & 7.00 & 1.40 & 2.80 & 4.19 & 5.59 & 5.89 & 4.56 & 4.86 & 4.75 & 4.75 & 6.35 \\
\hline 14.00 & 7.00 & 1.40 & 2.80 & 4.19 & 5.59 & 5.90 & 4.67 & 5.08 & 4.95 & 4.81 & 6.35 \\
\hline 15.00 & 7.00 & 1.40 & 2.80 & 4.19 & 5.59 & 5.91 & 4.78 & 5.30 & 5.16 & 4.88 & 6.36 \\
\hline 16.00 & 7.00 & 1.40 & 2.80 & 4.19 & 5.59 & 5.92 & 4.89 & 5.53 & 5.36 & 4.94 & 6.36 \\
\hline 17.00 & 7.00 & 1.40 & 2.80 & 4.19 & 5.59 & 5.93 & 5.00 & 5.75 & 5.56 & 5.00 & 6.36 \\
\hline 18.00 & 7.00 & 1.40 & 2.80 & 4.19 & 5.59 & 5.93 & 5.11 & 5.98 & 5.76 & 5.06 & 6.36 \\
\hline 19.00 & 7.00 & 1.40 & 2.80 & 4.19 & 5.59 & 5.94 & 5.22 & 6.20 & 5.97 & 5.13 & 6.37 \\
\hline 20.00 & 7.00 & 1.40 & 2.80 & 4.19 & 5.59 & 5.95 & 5.33 & 6.43 & 6.17 & 5.19 & 6.37 \\
\hline 21.00 & 7.00 & 1.40 & 2.80 & 4.19 & 5.59 & 5.96 & 5.44 & 6.65 & 6.37 & 5.25 & 6.37 \\
\hline 22.00 & 7.00 & 1.40 & 2.80 & 4.19 & 5.59 & 5.97 & 5.56 & 6.88 & 6.57 & 5.31 & 6.37 \\
\hline 23.00 & 7.00 & 1.40 & 2.80 & 4.19 & 5.59 & 5.98 & 5.67 & 7.10 & 6.78 & 5.38 & 6.38 \\
\hline 24.00 & 7.00 & 1.40 & 2.80 & 4.19 & 5.59 & 5.98 & 5.78 & 7.32 & 6.98 & 5.44 & 6.38 \\
\hline 25.00 & 7.00 & 1.40 & 2.80 & 4.19 & 5.59 & 5.99 & 5.89 & 7.55 & 7.18 & 5.50 & 6.38 \\
\hline 26.00 & 7.00 & 1.40 & 2.80 & 4.19 & 5.59 & 6.00 & 6.00 & 7.77 & 7.38 & 5.56 & 6.38 \\
\hline 27.00 & 7.00 & 1.40 & 2.80 & 4.19 & 5.59 & 6.01 & 6.11 & 8.00 & 7.59 & 5.63 & 6.39 \\
\hline 28.00 & 7.00 & 1.40 & 2.80 & 4.19 & 5.59 & 6.02 & 6.22 & 8.22 & 7.79 & 5.69 & 6.39 \\
\hline 29.00 & 7.00 & 1.40 & 2.80 & 4.19 & 5.59 & 6.02 & 6.33 & 8.45 & 7.99 & 5.75 & 6.39 \\
\hline 30.00 & 7.00 & 1.40 & 2.80 & 4.19 & 5.59 & 6.03 & 6.44 & 8.67 & 8.19 & 5.81 & 6.39 \\
\hline
\end{tabular}


Table 3.5: Risk for different values of $\Delta^{2}$ at $p=6$ and $k=d=0.1,0.5$ and 0.9

\begin{tabular}{|c|c|c|c|c|c|c|c|c|c|c|c|}
\hline \multirow[t]{2}{*}{$\Delta^{2}$} & \multirow[t]{2}{*}{ LSE } & \multicolumn{4}{|c|}{ LASSO } & \multicolumn{3}{|c|}{ Ridge } & \multicolumn{3}{|c|}{ Liu } \\
\hline & & $q=1$ & $q=3$ & $q=5$ & $q=6$ & $k=0.1$ & $k=0.5$ & $k=0.9$ & $d=0.1$ & $d=0.5$ & $d=0.9$ \\
\hline 0.00 & 6.00 & 1.53 & 3.06 & 4.58 & 5.35 & 4.96 & 2.67 & 1.66 & 1.82 & 3.38 & 5.42 \\
\hline 1.00 & 6.00 & 1.53 & 3.06 & 4.58 & 5.35 & 4.97 & 2.78 & 1.89 & 2.02 & 3.44 & 5.42 \\
\hline 2.00 & 6.00 & 1.53 & 3.06 & 4.58 & 5.35 & 4.98 & 2.89 & 2.11 & 2.22 & 3.50 & 5.42 \\
\hline 3.00 & 6.00 & 1.53 & 3.06 & 4.58 & 5.35 & 4.98 & 3.00 & 2.34 & 2.42 & 3.56 & 5.42 \\
\hline 4.00 & 6.00 & 1.53 & 3.06 & 4.58 & 5.35 & 4.99 & 3.11 & 2.56 & 2.63 & 3.63 & 5.43 \\
\hline 5.00 & 6.00 & 1.53 & 3.06 & 4.58 & 5.35 & 5.00 & 3.22 & 2.78 & 2.83 & 3.69 & 5.43 \\
\hline 6.00 & 6.00 & 1.53 & 3.06 & 4.58 & 5.35 & 5.01 & 3.33 & 3.01 & 3.03 & 3.75 & 5.43 \\
\hline 7.00 & 6.00 & 1.53 & 3.06 & 4.58 & 5.35 & 5.02 & 3.44 & 3.23 & 3.23 & 3.81 & 5.43 \\
\hline 8.00 & 6.00 & 1.53 & 3.06 & 4.58 & 5.35 & 5.02 & 3.56 & 3.46 & 3.44 & 3.88 & 5.44 \\
\hline 9.00 & 6.00 & 1.53 & 3.06 & 4.58 & 5.35 & 5.03 & 3.67 & 3.68 & 3.64 & 3.94 & 5.44 \\
\hline 10.00 & 6.00 & 1.53 & 3.06 & 4.58 & 5.35 & 5.04 & 3.78 & 3.91 & 3.84 & 4.00 & 5.44 \\
\hline 11.00 & 6.00 & 1.53 & 3.06 & 4.58 & 5.35 & 5.05 & 3.89 & 4.13 & 4.04 & 4.06 & 5.44 \\
\hline 12.00 & 6.00 & 1.53 & 3.06 & 4.58 & 5.35 & 5.06 & 4.00 & 4.35 & 4.25 & 4.13 & 5.45 \\
\hline 13.00 & 6.00 & 1.53 & 3.06 & 4.58 & 5.35 & 5.07 & 4.11 & 4.58 & 4.45 & 4.19 & 5.45 \\
\hline 14.00 & 6.00 & 1.53 & 3.06 & 4.58 & 5.35 & 5.07 & 4.22 & 4.80 & 4.65 & 4.25 & 5.45 \\
\hline 15.00 & 6.00 & 1.53 & 3.06 & 4.58 & 5.35 & 5.08 & 4.33 & 5.03 & 4.85 & 4.31 & 5.45 \\
\hline 16.00 & 6.00 & 1.53 & 3.06 & 4.58 & 5.35 & 5.09 & 4.44 & 5.25 & 5.06 & 4.38 & 5.46 \\
\hline 17.00 & 6.00 & 1.53 & 3.06 & 4.58 & 5.35 & 5.10 & 4.56 & 5.48 & 5.26 & 4.44 & 5.46 \\
\hline 18.00 & 6.00 & 1.53 & 3.06 & 4.58 & 5.35 & 5.11 & 4.67 & 5.70 & 5.46 & 4.50 & 5.46 \\
\hline 19.00 & 6.00 & 1.53 & 3.06 & 4.58 & 5.35 & 5.12 & 4.78 & 5.93 & 5.66 & 4.56 & 5.46 \\
\hline 20.00 & 6.00 & 1.53 & 3.06 & 4.58 & 5.35 & 5.12 & 4.89 & 6.15 & 5.87 & 4.63 & 5.47 \\
\hline 21.00 & 6.00 & 1.53 & 3.06 & 4.58 & 5.35 & 5.13 & 5.00 & 6.37 & 6.07 & 4.69 & 5.47 \\
\hline 22.00 & 6.00 & 1.53 & 3.06 & 4.58 & 5.35 & 5.14 & 5.11 & 6.60 & 6.27 & 4.75 & 5.47 \\
\hline 23.00 & 6.00 & 1.53 & 3.06 & 4.58 & 5.35 & 5.15 & 5.22 & 6.82 & 6.47 & 4.81 & 5.47 \\
\hline 24.00 & 6.00 & 1.53 & 3.06 & 4.58 & 5.35 & 5.16 & 5.33 & 7.05 & 6.68 & 4.88 & 5.48 \\
\hline 25.00 & 6.00 & 1.53 & 3.06 & 4.58 & 5.35 & 5.17 & 5.44 & 7.27 & 6.88 & 4.94 & 5.48 \\
\hline 26.00 & 6.00 & 1.53 & 3.06 & 4.58 & 5.35 & 5.17 & 5.56 & 7.50 & 7.08 & 5.00 & 5.48 \\
\hline 27.00 & 6.00 & 1.53 & 3.06 & 4.58 & 5.35 & 5.18 & 5.67 & 7.72 & 7.28 & 5.06 & 5.48 \\
\hline 28.00 & 6.00 & 1.53 & 3.06 & 4.58 & 5.35 & 5.19 & 5.78 & 7.94 & 7.49 & 5.13 & 5.49 \\
\hline 29.00 & 6.00 & 1.53 & 3.06 & 4.58 & 5.35 & 5.20 & 5.89 & 8.17 & 7.69 & 5.19 & 5.49 \\
\hline 30.00 & 6.00 & 1.53 & 3.06 & 4.58 & 5.35 & 5.21 & 6.00 & 8.39 & 7.89 & 5.25 & 5.49 \\
\hline
\end{tabular}


Table 3.6: Risk for different values of $\Delta^{2}$ at $p=8$ and $k=d=0.1,0.5$ and 0.9

\begin{tabular}{|c|c|c|c|c|c|c|c|c|c|c|c|c|}
\hline \multirow[t]{2}{*}{$\Delta^{2}$} & \multirow[t]{2}{*}{ LSE } & \multicolumn{5}{|c|}{ LASSO } & \multicolumn{3}{|c|}{ Ridge } & \multicolumn{3}{|c|}{ Liu } \\
\hline & & $q=1$ & $q=3$ & $q=5$ & $q=7$ & $q=8$ & $k=0.1$ & $k=0.5$ & $k=0.9$ & $d=0.1$ & $d=0.5$ & $d=0.9$ \\
\hline 0.00 & 8.00 & 1.29 & 2.58 & 3.87 & 5.16 & 5.80 & 6.61 & 3.56 & 2.22 & 2.42 & 4.50 & 7.22 \\
\hline 1.00 & 8.00 & 1.29 & 2.58 & 3.87 & 5.16 & 5.80 & 6.62 & 3.67 & 2.44 & 2.62 & 4.56 & 7.22 \\
\hline 2.00 & 8.00 & 1.29 & 2.58 & 3.87 & 5.16 & 5.80 & 6.63 & 3.78 & 2.66 & 2.83 & 4.63 & 7.23 \\
\hline 3.00 & 8.00 & 1.29 & 2.58 & 3.87 & 5.16 & 5.80 & 6.64 & 3.89 & 2.89 & 3.03 & 4.69 & 7.23 \\
\hline 4.00 & 8.00 & 1.29 & 2.58 & 3.87 & 5.16 & 5.80 & 6.64 & 4.00 & 3.11 & 3.23 & 4.75 & 7.23 \\
\hline 5.00 & 8.00 & 1.29 & 2.58 & 3.87 & 5.16 & 5.80 & 6.65 & 4.11 & 3.34 & 3.43 & 4.81 & 7.23 \\
\hline 6.00 & 8.00 & 1.29 & 2.58 & 3.87 & 5.16 & 5.80 & 6.66 & 4.22 & 3.56 & 3.64 & 4.88 & 7.24 \\
\hline 7.00 & 8.00 & 1.29 & 2.58 & 3.87 & 5.16 & 5.80 & 6.67 & 4.33 & 3.79 & 3.84 & 4.94 & 7.24 \\
\hline 8.00 & 8.00 & 1.29 & 2.58 & 3.87 & 5.16 & 5.80 & 6.68 & 4.44 & 4.01 & 4.04 & 5.00 & 7.24 \\
\hline 9.00 & 8.00 & 1.29 & 2.58 & 3.87 & 5.16 & 5.80 & 6.69 & 4.56 & 4.24 & 4.24 & 5.06 & 7.24 \\
\hline 10.00 & 8.00 & 1.29 & 2.58 & 3.87 & 5.16 & 5.80 & 6.69 & 4.67 & 4.46 & 4.45 & 5.13 & 7.25 \\
\hline 11.00 & 8.00 & 1.29 & 2.58 & 3.87 & 5.16 & 5.80 & 6.70 & 4.78 & 4.68 & 4.65 & 5.19 & 7.25 \\
\hline 12.00 & 8.00 & 1.29 & 2.58 & 3.87 & 5.16 & 5.80 & 6.71 & 4.89 & 4.91 & 4.85 & 5.25 & 7.25 \\
\hline 13.00 & 8.00 & 1.29 & 2.58 & 3.87 & 5.16 & 5.80 & 6.72 & 5.00 & 5.13 & 5.05 & 5.31 & 7.25 \\
\hline 14.00 & 8.00 & 1.29 & 2.58 & 3.87 & 5.16 & 5.80 & 6.73 & 5.11 & 5.36 & 5.26 & 5.38 & 7.26 \\
\hline 15.00 & 8.00 & 1.29 & 2.58 & 3.87 & 5.16 & 5.80 & 6.74 & 5.22 & 5.58 & 5.46 & 5.44 & 7.26 \\
\hline 16.00 & 8.00 & 1.29 & 2.58 & 3.87 & 5.16 & 5.80 & 6.74 & 5.33 & 5.81 & 5.66 & 5.50 & 7.26 \\
\hline 17.00 & 8.00 & 1.29 & 2.58 & 3.87 & 5.16 & 5.80 & 6.75 & 5.44 & 6.03 & 5.86 & 5.56 & 7.26 \\
\hline 18.00 & 8.00 & 1.29 & 2.58 & 3.87 & 5.16 & 5.80 & 6.76 & 5.56 & 6.25 & 6.07 & 5.63 & 7.27 \\
\hline 19.00 & 8.00 & 1.29 & 2.58 & 3.87 & 5.16 & 5.80 & 6.77 & 5.67 & 6.48 & 6.27 & 5.69 & 7.27 \\
\hline 20.00 & 8.00 & 1.29 & 2.58 & 3.87 & 5.16 & 5.80 & 6.78 & 5.78 & 6.70 & 6.47 & 5.75 & 7.27 \\
\hline 21.00 & 8.00 & 1.29 & 2.58 & 3.87 & 5.16 & 5.80 & 6.79 & 5.89 & 6.93 & 6.67 & 5.81 & 7.27 \\
\hline 22.00 & 8.00 & 1.29 & 2.58 & 3.87 & 5.16 & 5.80 & 6.79 & 6.00 & 7.15 & 6.88 & 5.88 & 7.28 \\
\hline 23.00 & 8.00 & 1.29 & 2.58 & 3.87 & 5.16 & 5.80 & 6.80 & 6.11 & 7.38 & 7.08 & 5.94 & 7.28 \\
\hline 24.00 & 8.00 & 1.29 & 2.58 & 3.87 & 5.16 & 5.80 & 6.81 & 6.22 & 7.60 & 7.28 & 6.00 & 7.28 \\
\hline 25.00 & 8.00 & 1.29 & 2.58 & 3.87 & 5.16 & 5.80 & 6.82 & 6.33 & 7.83 & 7.48 & 6.06 & 7.28 \\
\hline 26.00 & 8.00 & 1.29 & 2.58 & 3.87 & 5.16 & 5.80 & 6.83 & 6.44 & 8.05 & 7.69 & 6.13 & 7.29 \\
\hline 27.00 & 8.00 & 1.29 & 2.58 & 3.87 & 5.16 & 5.80 & 6.83 & 6.56 & 8.27 & 7.89 & 6.19 & 7.29 \\
\hline 28.00 & 8.00 & 1.29 & 2.58 & 3.87 & 5.16 & 5.80 & 6.84 & 6.67 & 8.50 & 8.09 & 6.25 & 7.29 \\
\hline 29.00 & 8.00 & 1.29 & 2.58 & 3.87 & 5.16 & 5.80 & 6.85 & 6.78 & 8.72 & 8.29 & 6.31 & 7.29 \\
\hline 30.00 & 8.00 & 1.29 & 2.58 & 3.87 & 5.16 & 5.80 & 6.86 & 6.89 & 8.95 & 8.50 & 6.38 & 7.30 \\
\hline
\end{tabular}


Table 3.7: Risk for different values of $\Delta^{2}$ at $p=9$ and $k=d=0.1,0.5$ and 0.9

\begin{tabular}{|c|c|c|c|c|c|c|c|c|c|c|c|c|}
\hline \multirow[t]{2}{*}{$\Delta^{2}$} & \multirow[t]{2}{*}{ LSE } & \multicolumn{5}{|c|}{ LASSO } & \multicolumn{3}{|c|}{ Ridge } & \multicolumn{3}{|c|}{ Liu } \\
\hline & & $q=1$ & $q=3$ & $q=5$ & $q=7$ & $q=9$ & $k=0.1$ & $k=0.5$ & $k=0.9$ & $d=0.1$ & $d=0.5$ & $d=0.9$ \\
\hline 0.00 & 9.00 & 1.20 & 2.40 & 3.60 & 4.80 & 5.99 & 7.44 & 4.00 & 2.49 & 2.72 & 5.06 & 8.12 \\
\hline 1.00 & 9.00 & 1.20 & 2.40 & 3.60 & 4.80 & 5.99 & 7.45 & 4.11 & 2.72 & 2.93 & 5.13 & 8.13 \\
\hline 2.00 & 9.00 & 1.20 & 2.40 & 3.60 & 4.80 & 5.99 & 7.45 & 4.22 & 2.94 & 3.13 & 5.19 & 8.13 \\
\hline 3.00 & 9.00 & 1.20 & 2.40 & 3.60 & 4.80 & 5.99 & 7.46 & 4.33 & 3.17 & 3.33 & 5.25 & 8.13 \\
\hline 4.00 & 9.00 & 1.20 & 2.40 & 3.60 & 4.80 & 5.99 & 7.47 & 4.44 & 3.39 & 3.53 & 5.31 & 8.13 \\
\hline 5.00 & 9.00 & 1.20 & 2.40 & 3.60 & 4.80 & 5.99 & 7.48 & 4.56 & 3.61 & 3.74 & 5.38 & 8.14 \\
\hline 6.00 & 9.00 & 1.20 & 2.40 & 3.60 & 4.80 & 5.99 & 7.49 & 4.67 & 3.84 & 3.94 & 5.44 & 8.14 \\
\hline 7.00 & 9.00 & 1.20 & 2.40 & 3.60 & 4.80 & 5.99 & 7.50 & 4.78 & 4.06 & 4.14 & 5.50 & 8.14 \\
\hline 8.00 & 9.00 & 1.20 & 2.40 & 3.60 & 4.80 & 5.99 & 7.50 & 4.89 & 4.29 & 4.34 & 5.56 & 8.14 \\
\hline 9.00 & 9.00 & 1.20 & 2.40 & 3.60 & 4.80 & 5.99 & 7.51 & 5.00 & 4.51 & 4.55 & 5.63 & 8.15 \\
\hline 10.00 & 9.00 & 1.20 & 2.40 & 3.60 & 4.80 & 5.99 & 7.52 & 5.11 & 4.74 & 4.75 & 5.69 & 8.15 \\
\hline 11.00 & 9.00 & 1.20 & 2.40 & 3.60 & 4.80 & 5.99 & 7.53 & 5.22 & 4.96 & 4.95 & 5.75 & 8.15 \\
\hline 12.00 & 9.00 & 1.20 & 2.40 & 3.60 & 4.80 & 5.99 & 7.54 & 5.33 & 5.19 & 5.15 & 5.81 & 8.15 \\
\hline 13.00 & 9.00 & 1.20 & 2.40 & 3.60 & 4.80 & 5.99 & 7.55 & 5.44 & 5.41 & 5.36 & 5.88 & 8.16 \\
\hline 14.00 & 9.00 & 1.20 & 2.40 & 3.60 & 4.80 & 5.99 & 7.55 & 5.56 & 5.63 & 5.56 & 5.94 & 8.16 \\
\hline 15.00 & 9.00 & 1.20 & 2.40 & 3.60 & 4.80 & 5.99 & 7.56 & 5.67 & 5.86 & 5.76 & 6.00 & 8.16 \\
\hline 16.00 & 9.00 & 1.20 & 2.40 & 3.60 & 4.80 & 5.99 & 7.57 & 5.78 & 6.08 & 5.96 & 6.06 & 8.16 \\
\hline 17.00 & 9.00 & 1.20 & 2.40 & 3.60 & 4.80 & 5.99 & 7.58 & 5.89 & 6.31 & 6.17 & 6.13 & 8.17 \\
\hline 18.00 & 9.00 & 1.20 & 2.40 & 3.60 & 4.80 & 5.99 & 7.59 & 6.00 & 6.53 & 6.37 & 6.19 & 8.17 \\
\hline 19.00 & 9.00 & 1.20 & 2.40 & 3.60 & 4.80 & 5.99 & 7.60 & 6.11 & 6.76 & 6.57 & 6.25 & 8.17 \\
\hline 20.00 & 9.00 & 1.20 & 2.40 & 3.60 & 4.80 & 5.99 & 7.60 & 6.22 & 6.98 & 6.77 & 6.31 & 8.17 \\
\hline 21.00 & 9.00 & 1.20 & 2.40 & 3.60 & 4.80 & 5.99 & 7.61 & 6.33 & 7.20 & 6.98 & 6.38 & 8.18 \\
\hline 22.00 & 9.00 & 1.20 & 2.40 & 3.60 & 4.80 & 5.99 & 7.62 & 6.44 & 7.43 & 7.18 & 6.44 & 8.18 \\
\hline 23.00 & 9.00 & 1.20 & 2.40 & 3.60 & 4.80 & 5.99 & 7.63 & 6.56 & 7.65 & 7.38 & 6.50 & 8.18 \\
\hline 24.00 & 9.00 & 1.20 & 2.40 & 3.60 & 4.80 & 5.99 & 7.64 & 6.67 & 7.88 & 7.58 & 6.56 & 8.18 \\
\hline 25.00 & 9.00 & 1.20 & 2.40 & 3.60 & 4.80 & 5.99 & 7.64 & 6.78 & 8.10 & 7.79 & 6.63 & 8.19 \\
\hline 26.00 & 9.00 & 1.20 & 2.40 & 3.60 & 4.80 & 5.99 & 7.65 & 6.89 & 8.33 & 7.99 & 6.69 & 8.19 \\
\hline 27.00 & 9.00 & 1.20 & 2.40 & 3.60 & 4.80 & 5.99 & 7.66 & 7.00 & 8.55 & 8.19 & 6.75 & 8.19 \\
\hline 28.00 & 9.00 & 1.20 & 2.40 & 3.60 & 4.80 & 5.99 & 7.67 & 7.11 & 8.78 & 8.39 & 6.81 & 8.19 \\
\hline 29.00 & 9.00 & 1.20 & 2.40 & 3.60 & 4.80 & 5.99 & 7.68 & 7.22 & 9.00 & 8.60 & 6.88 & 8.20 \\
\hline 30.00 & 9.00 & 1.20 & 2.40 & 3.60 & 4.80 & 5.99 & 7.69 & 7.33 & 9.22 & 8.80 & 6.94 & 8.20 \\
\hline
\end{tabular}


Table 3.8: Risk for different values of $\Delta^{2}$ at $p=10$ and $k=d=0.1,0.5$ and 0.9

\begin{tabular}{|c|c|c|c|c|c|c|c|c|c|c|c|c|c|}
\hline \multirow[t]{2}{*}{$\Delta^{2}$} & \multirow[t]{2}{*}{ LSE } & \multicolumn{6}{|c|}{ LASSO } & \multicolumn{3}{|c|}{ Ridge } & \multicolumn{3}{|c|}{ Liu } \\
\hline & & $q=1$ & $q=3$ & $q=5$ & $q=7$ & $q=9$ & $q=10$ & $k=0.1$ & $k=0.5$ & $k=0.9$ & $d=0.1$ & $d=0.5$ & $\mathrm{~d}=0.9$ \\
\hline 0.00 & 10.00 & 1.12 & 2.24 & 3.36 & 4.48 & 5.61 & 6.17 & 8.26 & 4.44 & 2.77 & 3.03 & 5.63 & 9.03 \\
\hline 1.00 & 10.00 & 1.12 & 2.24 & 3.36 & 4.48 & 5.61 & 6.17 & 8.27 & 4.56 & 2.99 & 3.23 & 5.69 & 9.03 \\
\hline 2.00 & 10.00 & 1.12 & 2.24 & 3.36 & 4.48 & 5.61 & 6.17 & 8.28 & 4.67 & 3.22 & 3.43 & 5.75 & 9.03 \\
\hline 3.00 & 10.00 & 1.12 & 2.24 & 3.36 & 4.48 & 5.61 & 6.17 & 8.29 & 4.78 & 3.44 & 3.63 & 5.81 & 9.03 \\
\hline 4.00 & 10.00 & 1.12 & 2.24 & 3.36 & 4.48 & 5.61 & 6.17 & 8.30 & 4.89 & 3.67 & 3.84 & 5.88 & 9.04 \\
\hline 5.00 & 10.00 & 1.12 & 2.24 & 3.36 & 4.48 & 5.61 & 6.17 & 8.31 & 5.00 & 3.89 & 4.04 & 5.94 & 9.04 \\
\hline 6.00 & 10.00 & 1.12 & 2.24 & 3.36 & 4.48 & 5.61 & 6.17 & 8.31 & 5.11 & 4.12 & 4.24 & 6.00 & 9.04 \\
\hline 7.00 & 10.00 & 1.12 & 2.24 & 3.36 & 4.48 & 5.61 & 6.17 & 8.32 & 5.22 & 4.34 & 4.44 & 6.06 & 9.04 \\
\hline 8.00 & 10.00 & 1.12 & 2.24 & 3.36 & 4.48 & 5.61 & 6.17 & 8.33 & 5.33 & 4.57 & 4.65 & 6.13 & 9.05 \\
\hline 9.00 & 10.00 & 1.12 & 2.24 & 3.36 & 4.48 & 5.61 & 6.17 & 8.34 & 5.44 & 4.79 & 4.85 & 6.19 & 9.05 \\
\hline 10.00 & 10.00 & 1.12 & 2.24 & 3.36 & 4.48 & 5.61 & 6.17 & 8.35 & 5.56 & 5.01 & 5.05 & 6.25 & 9.05 \\
\hline 11.00 & 10.00 & 1.12 & 2.24 & 3.36 & 4.48 & 5.61 & 6.17 & 8.36 & 5.67 & 5.24 & 5.25 & 6.31 & 9.05 \\
\hline 12.00 & 10.00 & 1.12 & 2.24 & 3.36 & 4.48 & 5.61 & 6.17 & 8.36 & 5.78 & 5.46 & 5.46 & 6.38 & 9.06 \\
\hline 13.00 & 10.00 & 1.12 & 2.24 & 3.36 & 4.48 & 5.61 & 6.17 & 8.37 & 5.89 & 5.69 & 5.66 & 6.44 & 9.06 \\
\hline 14.00 & 10.00 & 1.12 & 2.24 & 3.36 & 4.48 & 5.61 & 6.17 & 8.38 & 6.00 & 5.91 & 5.86 & 6.50 & 9.06 \\
\hline 15.00 & 10.00 & 1.12 & 2.24 & 3.36 & 4.48 & 5.61 & 6.17 & 8.39 & 6.11 & 6.14 & 6.06 & 6.56 & 9.06 \\
\hline 16.00 & 10.00 & 1.12 & 2.24 & 3.36 & 4.48 & 5.61 & 6.17 & 8.40 & 6.22 & 6.36 & 6.27 & 6.63 & 9.07 \\
\hline 17.00 & 10.00 & 1.12 & 2.24 & 3.36 & 4.48 & 5.61 & 6.17 & 8.40 & 6.33 & 6.58 & 6.47 & 6.69 & 9.07 \\
\hline 18.00 & 10.00 & 1.12 & 2.24 & 3.36 & 4.48 & 5.61 & 6.17 & 8.41 & 6.44 & 6.81 & 6.67 & 6.75 & 9.07 \\
\hline 19.00 & 10.00 & 1.12 & 2.24 & 3.36 & 4.48 & 5.61 & 6.17 & 8.42 & 6.56 & 7.03 & 6.87 & 6.81 & 9.07 \\
\hline 20.00 & 10.00 & 1.12 & 2.24 & 3.36 & 4.48 & 5.61 & 6.17 & 8.43 & 6.67 & 7.26 & 7.08 & 6.88 & 9.08 \\
\hline 21.00 & 10.00 & 1.12 & 2.24 & 3.36 & 4.48 & 5.61 & 6.17 & 8.44 & 6.78 & 7.48 & 7.28 & 6.94 & 9.08 \\
\hline 22.00 & 10.00 & 1.12 & 2.24 & 3.36 & 4.48 & 5.61 & 6.17 & 8.45 & 6.89 & 7.71 & 7.48 & 7.00 & 9.08 \\
\hline 23.00 & 10.00 & 1.12 & 2.24 & 3.36 & 4.48 & 5.61 & 6.17 & 8.45 & 7.00 & 7.93 & 7.68 & 7.06 & 9.08 \\
\hline 24.00 & 10.00 & 1.12 & 2.24 & 3.36 & 4.48 & 5.61 & 6.17 & 8.46 & 7.11 & 8.16 & 7.89 & 7.13 & 9.09 \\
\hline 25.00 & 10.00 & 1.12 & 2.24 & 3.36 & 4.48 & 5.61 & 6.17 & 8.47 & 7.22 & 8.38 & 8.09 & 7.19 & 9.09 \\
\hline 26.00 & 10.00 & 1.12 & 2.24 & 3.36 & 4.48 & 5.61 & 6.17 & 8.48 & 7.33 & 8.60 & 8.29 & 7.25 & 9.09 \\
\hline 27.00 & 10.00 & 1.12 & 2.24 & 3.36 & 4.48 & 5.61 & 6.17 & 8.49 & 7.44 & 8.83 & 8.49 & 7.31 & 9.09 \\
\hline 28.00 & 10.00 & 1.12 & 2.24 & 3.36 & 4.48 & 5.61 & 6.17 & 8.50 & 7.56 & 9.05 & 8.70 & 7.38 & 9.10 \\
\hline 29.00 & 10.00 & 1.12 & 2.24 & 3.36 & 4.48 & 5.61 & 6.17 & 8.50 & 7.67 & 9.28 & 8.90 & 7.44 & 9.10 \\
\hline 30.00 & 10.00 & 1.12 & 2.24 & 3.36 & 4.48 & 5.61 & 6.17 & 8.51 & 7.78 & 9.50 & 9.10 & 7.50 & 9.10 \\
\hline
\end{tabular}


Table 3.9: Efficiency for different values of $\Delta^{2}$ at $p=3$ and $k=d=0.1,0.5$ and 0.9

\begin{tabular}{|c|c|c|c|c|c|c|c|c|c|c|}
\hline \multirow{2}{*}{$\Delta^{2}$} & \multirow[t]{2}{*}{ LSE } & \multicolumn{3}{|c|}{ LASSO } & \multicolumn{3}{|l|}{ Ridge } & \multicolumn{3}{|l|}{ Liu } \\
\hline & & $q=1$ & $q=2$ & $q=3$ & $k=0.1$ & $k=0.5$ & $k=0.9$ & $d=0.1$ & $d=0.5$ & $d=0.9$ \\
\hline 0.00 & 1.00 & 1.41 & 0.94 & 0.70 & 1.21 & 2.25 & 3.61 & 3.31 & 1.78 & 1.11 \\
\hline 1.00 & 1.00 & 1.41 & 0.94 & 0.70 & 1.21 & 2.08 & 2.84 & 2.70 & 1.71 & 1.11 \\
\hline 2.00 & 1.00 & 1.41 & 0.94 & 0.70 & 1.20 & 1.93 & 2.34 & 2.29 & 1.66 & 1.11 \\
\hline $\begin{array}{l}3.00 \\
4.00\end{array}$ & $\begin{array}{l}1.00 \\
1.00\end{array}$ & $\begin{array}{l}1.41 \\
1.41\end{array}$ & $\begin{array}{l}0.94 \\
0.94\end{array}$ & $\begin{array}{l}0.70 \\
0.70\end{array}$ & $\begin{array}{l}1.20 \\
1.19\end{array}$ & $\begin{array}{l}1.80 \\
1.69\end{array}$ & $\begin{array}{l}1.99 \\
1.74\end{array}$ & $\begin{array}{l}1.98 \\
1.75\end{array}$ & $\begin{array}{l}1.60 \\
1.55\end{array}$ & $\begin{array}{l}1.10 \\
1.10\end{array}$ \\
\hline 5.00 & 1.00 & 1.41 & 0.94 & 0.70 & 1.19 & 1.59 & 1.54 & 1.56 & 1.50 & 1.10 \\
\hline 6.00 & 1.00 & 1.41 & 0.94 & 0.70 & 1.19 & 1.50 & 1.38 & 1.41 & 1.45 & 1.10 \\
\hline 7.00 & 1.00 & 1.41 & 0.94 & 0.70 & 1.18 & 1.42 & 1.25 & 1.29 & 1.41 & 1.10 \\
\hline 8.00 & 1.00 & 1.41 & 0.94 & 0.70 & 1.18 & 1.35 & 1.14 & 1.19 & 1.37 & 1.10 \\
\hline 9.00 & 1.00 & 1.41 & 0.94 & 0.70 & 1.17 & 1.29 & 1.05 & 1.10 & 1.33 & 1.10 \\
\hline 10.00 & 1.00 & 1.41 & 0.94 & 0.70 & 1.17 & 1.23 & 0.98 & 1.02 & 1.30 & 1.10 \\
\hline 11.00 & 1.00 & 1.41 & 0.94 & 0.70 & 1.17 & 1.17 & 0.91 & 0.96 & 1.26 & 1.10 \\
\hline 12.00 & 1.00 & 1.41 & 0.94 & 0.70 & 1.16 & 1.13 & 0.85 & 0.90 & 1.23 & 1.10 \\
\hline 13.00 & 1.00 & 1.41 & 0.94 & 0.70 & 1.16 & 1.08 & 0.80 & 0.85 & 1.20 & 1.09 \\
\hline 14.00 & 1.00 & 1.41 & 0.94 & 0.70 & 1.16 & 1.04 & 0.76 & 0.80 & 1.17 & 1.09 \\
\hline 15.00 & 1.00 & 1.41 & 0.94 & 0.70 & 1.15 & 1.00 & 0.71 & 0.76 & 1.14 & 1.09 \\
\hline 16.00 & 1.00 & 1.41 & 0.94 & 0.70 & 1.15 & 0.96 & 0.68 & 0.72 & 1.12 & 1.09 \\
\hline 17.00 & 1.00 & 1.41 & 0.94 & 0.70 & 1.15 & 0.93 & 0.65 & 0.69 & 1.09 & 1.09 \\
\hline 18.00 & 1.00 & 1.41 & 0.94 & 0.70 & 1.14 & 0.90 & 0.62 & 0.66 & 1.07 & 1.09 \\
\hline 19.00 & 1.00 & 1.41 & 0.94 & 0.70 & 1.14 & 0.87 & 0.59 & 0.63 & 1.04 & 1.09 \\
\hline 20.00 & 1.00 & 1.41 & 0.94 & 0.70 & 1.13 & 0.84 & 0.56 & 0.61 & 1.02 & 1.09 \\
\hline 21.00 & 1.00 & 1.41 & 0.94 & 0.70 & 1.13 & 0.82 & 0.54 & 0.58 & 1.00 & 1.09 \\
\hline 22.00 & 1.00 & 1.41 & 0.94 & 0.70 & 1.13 & 0.79 & 0.52 & 0.56 & 0.98 & 1.09 \\
\hline 23.00 & 1.00 & 1.41 & 0.94 & 0.70 & 1.12 & 0.77 & 0.50 & 0.54 & 0.96 & 1.08 \\
\hline 24.00 & 1.00 & 1.41 & 0.94 & 0.70 & 1.12 & 0.75 & 0.48 & 0.52 & 0.94 & 1.08 \\
\hline 25.00 & 1.00 & 1.41 & 0.94 & 0.70 & 1.12 & 0.73 & 0.47 & 0.50 & 0.92 & 1.08 \\
\hline 26.00 & 1.00 & 1.41 & 0.94 & 0.70 & 1.11 & 0.71 & 0.45 & 0.49 & 0.91 & 1.08 \\
\hline 27.00 & 1.00 & 1.41 & 0.94 & 0.70 & 1.11 & 0.69 & 0.44 & 0.47 & 0.89 & 1.08 \\
\hline 28.00 & 1.00 & 1.41 & 0.94 & 0.70 & 1.11 & 0.68 & 0.42 & 0.46 & 0.87 & 1.08 \\
\hline 29.00 & 1.00 & 1.41 & 0.94 & 0.70 & 1.10 & 0.66 & 0.41 & 0.44 & 0.86 & 1.08 \\
\hline 30.00 & 1.00 & 1.41 & 0.94 & 0.70 & 1.10 & 0.64 & 0.40 & 0.43 & 0.84 & 1.08 \\
\hline
\end{tabular}


Table 3.10: Efficiency for different values of $\Delta^{2}$ at $p=5$ and $k=d=0.1,0.5$ and 0.9

\begin{tabular}{|c|c|c|c|c|c|c|c|c|c|c|}
\hline \multirow[t]{2}{*}{$\Delta^{2}$} & \multirow[t]{2}{*}{ LSE } & \multicolumn{3}{|c|}{ LASSO } & \multicolumn{3}{|c|}{ Ridge } & \multicolumn{3}{|c|}{ Liu } \\
\hline & & $q=1$ & $q=3$ & $q=5$ & $k=0.1$ & $k=0.5$ & $k=0.9$ & $d=0.1$ & $d=0.5$ & $d=0.9$ \\
\hline 0.00 & 1.00 & 2.96 & 1.48 & 0.99 & 1.21 & 2.25 & 3.61 & 3.31 & 1.78 & 1.11 \\
\hline 1.00 & 1.00 & 2.96 & 1.48 & 0.99 & 1.21 & 2.14 & 3.11 & 2.92 & 1.74 & 1.11 \\
\hline 2.00 & 1.00 & 2.96 & 1.48 & 0.99 & 1.21 & 2.05 & 2.73 & 2.61 & 1.70 & 1.11 \\
\hline 3.00 & 1.00 & 2.96 & 1.48 & 0.99 & 1.20 & 1.96 & 2.43 & 2.36 & 1.67 & 1.11 \\
\hline 4.00 & 1.00 & 2.96 & 1.48 & 0.99 & 1.20 & 1.88 & 2.19 & 2.15 & 1.63 & 1.11 \\
\hline 5.00 & 1.00 & 2.96 & 1.48 & 0.99 & 1.20 & 1.80 & 1.99 & 1.98 & 1.60 & 1.10 \\
\hline 6.00 & 1.00 & 2.96 & 1.48 & 0.99 & 1.20 & 1.73 & 1.83 & 1.83 & 1.57 & 1.10 \\
\hline 7.00 & 1.00 & 2.96 & 1.48 & 0.99 & 1.19 & 1.67 & 1.69 & 1.71 & 1.54 & 1.10 \\
\hline 8.00 & 1.00 & 2.96 & 1.48 & 0.99 & 1.19 & 1.61 & 1.57 & 1.60 & 1.51 & 1.10 \\
\hline 9.00 & 1.00 & 2.96 & 1.48 & 0.99 & 1.19 & 1.55 & 1.47 & 1.50 & 1.48 & 1.10 \\
\hline 10.00 & 1.00 & 2.96 & 1.48 & 0.99 & 1.19 & 1.50 & 1.38 & 1.41 & 1.45 & 1.10 \\
\hline 11.00 & 1.00 & 2.96 & 1.48 & 0.99 & 1.18 & 1.45 & 1.30 & 1.34 & 1.43 & 1.10 \\
\hline 12.00 & 1.00 & 2.96 & 1.48 & 0.99 & 1.18 & 1.41 & 1.23 & 1.27 & 1.40 & 1.10 \\
\hline 13.00 & 1.00 & 2.96 & 1.48 & 0.99 & 1.18 & 1.36 & 1.16 & 1.21 & 1.38 & 1.10 \\
\hline 14.00 & 1.00 & 2.96 & 1.48 & 0.99 & 1.18 & 1.32 & 1.10 & 1.15 & 1.36 & 1.10 \\
\hline 15.00 & 1.00 & 2.96 & 1.48 & 0.99 & 1.17 & 1.29 & 1.05 & 1.10 & 1.33 & 1.10 \\
\hline 16.00 & 1.00 & 2.96 & 1.48 & 0.99 & 1.17 & 1.25 & 1.01 & 1.05 & 1.31 & 1.10 \\
\hline 17.00 & 1.00 & 2.96 & 1.48 & 0.99 & 1.17 & 1.22 & 0.96 & 1.01 & 1.29 & 1.10 \\
\hline 18.00 & 1.00 & 2.96 & 1.48 & 0.99 & 1.17 & 1.18 & 0.92 & 0.97 & 1.27 & 1.10 \\
\hline 19.00 & 1.00 & 2.96 & 1.48 & 0.99 & 1.17 & 1.15 & 0.89 & 0.93 & 1.25 & 1.10 \\
\hline 20.00 & 1.00 & 2.96 & 1.48 & 0.99 & 1.16 & 1.13 & 0.85 & 0.90 & 1.23 & 1.10 \\
\hline 21.00 & 1.00 & 2.96 & 1.48 & 0.99 & 1.16 & 1.10 & 0.82 & 0.87 & 1.21 & 1.10 \\
\hline 22.00 & 1.00 & 2.96 & 1.48 & 0.99 & 1.16 & 1.07 & 0.79 & 0.84 & 1.19 & 1.09 \\
\hline 23.00 & 1.00 & 2.96 & 1.48 & 0.99 & 1.16 & 1.05 & 0.76 & 0.81 & 1.18 & 1.09 \\
\hline 24.00 & 1.00 & 2.96 & 1.48 & 0.99 & 1.15 & 1.02 & 0.74 & 0.78 & 1.16 & 1.09 \\
\hline 25.00 & 1.00 & 2.96 & 1.48 & 0.99 & 1.15 & 1.00 & 0.71 & 0.76 & 1.14 & 1.09 \\
\hline 26.00 & 1.00 & 2.96 & 1.48 & 0.99 & 1.15 & 0.98 & 0.69 & 0.74 & 1.13 & 1.09 \\
\hline 27.00 & 1.00 & 2.96 & 1.48 & 0.99 & 1.15 & 0.96 & 0.67 & 0.72 & 1.11 & 1.09 \\
\hline 28.00 & 1.00 & 2.96 & 1.48 & 0.99 & 1.15 & 0.94 & 0.65 & 0.70 & 1.10 & 1.09 \\
\hline 29.00 & 1.00 & 2.96 & 1.48 & 0.99 & 1.14 & 0.92 & 0.63 & 0.68 & 1.08 & 1.09 \\
\hline 30.00 & 1.00 & 2.96 & 1.48 & 0.99 & 1.14 & 0.90 & 0.62 & 0.66 & 1.07 & 1.09 \\
\hline
\end{tabular}


Table 3.11: Efficiency for different values of $\Delta^{2}$ at $p=7$ and $k=d=0.1,0.5$ and 0.9

\begin{tabular}{|c|c|c|c|c|c|c|c|c|c|c|c|}
\hline \multirow[t]{2}{*}{$\Delta^{2}$} & \multirow[t]{2}{*}{ LSE } & \multicolumn{4}{|c|}{ LASSO } & \multicolumn{3}{|c|}{ Ridge } & \multicolumn{3}{|c|}{ Liu } \\
\hline & & $q=1$ & $q=3$ & $q=5$ & $q=7$ & $k=0.1$ & $k=0.5$ & $k=0.9$ & $d=0.1$ & $d=0.5$ & $d=0.9$ \\
\hline 0.00 & 1.00 & 5.01 & 2.50 & 1.67 & 1.25 & 1.21 & 2.25 & 3.61 & 3.31 & 1.78 & 1.11 \\
\hline 1.00 & 1.00 & 5.01 & 2.50 & 1.67 & 1.25 & 1.21 & 2.17 & 3.24 & 3.02 & 1.75 & 1.11 \\
\hline 2.00 & 1.00 & 5.01 & 2.50 & 1.67 & 1.25 & 1.21 & 2.10 & 2.93 & 2.78 & 1.72 & 1.11 \\
\hline 3.00 & 1.00 & 5.01 & 2.50 & 1.67 & 1.25 & 1.20 & 2.03 & 2.68 & 2.57 & 1.70 & 1.11 \\
\hline 4.00 & 1.00 & 5.01 & 2.50 & 1.67 & 1.25 & 1.20 & 1.97 & 2.47 & 2.39 & 1.67 & 1.11 \\
\hline 5.00 & 1.00 & 5.01 & 2.50 & 1.67 & 1.25 & 1.20 & 1.91 & 2.29 & 2.24 & 1.65 & 1.11 \\
\hline 6.00 & 1.00 & 5.01 & 2.50 & 1.67 & 1.25 & 1.20 & 1.85 & 2.13 & 2.10 & 1.62 & 1.11 \\
\hline 7.00 & 1.00 & 5.01 & 2.50 & 1.67 & 1.25 & 1.20 & 1.80 & 1.99 & 1.98 & 1.60 & 1.10 \\
\hline 8.00 & 1.00 & 5.01 & 2.50 & 1.67 & 1.25 & 1.20 & 1.75 & 1.87 & 1.87 & 1.58 & 1.10 \\
\hline 9.00 & 1.00 & 5.01 & 2.50 & 1.67 & 1.25 & 1.19 & 1.70 & 1.77 & 1.78 & 1.56 & 1.10 \\
\hline 10.00 & 1.00 & 5.01 & 2.50 & 1.67 & 1.25 & 1.19 & 1.66 & 1.67 & 1.69 & 1.53 & 1.10 \\
\hline 11.00 & 1.00 & 5.01 & 2.50 & 1.67 & 1.25 & 1.19 & 1.62 & 1.59 & 1.61 & 1.51 & 1.10 \\
\hline 12.00 & 1.00 & 5.01 & 2.50 & 1.67 & 1.25 & 1.19 & 1.58 & 1.51 & 1.54 & 1.49 & 1.10 \\
\hline 13.00 & 1.00 & 5.01 & 2.50 & 1.67 & 1.25 & 1.19 & 1.54 & 1.44 & 1.47 & 1.47 & 1.10 \\
\hline 14.00 & 1.00 & 5.01 & 2.50 & 1.67 & 1.25 & 1.19 & 1.50 & 1.38 & 1.41 & 1.45 & 1.10 \\
\hline 15.00 & 1.00 & 5.01 & 2.50 & 1.67 & 1.25 & 1.18 & 1.47 & 1.32 & 1.36 & 1.44 & 1.10 \\
\hline 16.00 & 1.00 & 5.01 & 2.50 & 1.67 & 1.25 & 1.18 & 1.43 & 1.27 & 1.31 & 1.42 & 1.10 \\
\hline 17.00 & 1.00 & 5.01 & 2.50 & 1.67 & 1.25 & 1.18 & 1.40 & 1.22 & 1.26 & 1.40 & 1.10 \\
\hline 18.00 & 1.00 & 5.01 & 2.50 & 1.67 & 1.25 & 1.18 & 1.37 & 1.17 & 1.21 & 1.38 & 1.10 \\
\hline 19.00 & 1.00 & 5.01 & 2.50 & 1.67 & 1.25 & 1.18 & 1.34 & 1.13 & 1.17 & 1.37 & 1.10 \\
\hline 20.00 & 1.00 & 5.01 & 2.50 & 1.67 & 1.25 & 1.18 & 1.31 & 1.09 & 1.13 & 1.35 & 1.10 \\
\hline 21.00 & & 5.01 & 2.50 & 1.67 & 1.25 & 1.17 & 1.29 & 1.05 & 1.10 & 1.33 & 1.10 \\
\hline 22.00 & 1.00 & 5.01 & 2.50 & 1.67 & 1.25 & 1.17 & 1.26 & 1.02 & 1.07 & 1.32 & 1.10 \\
\hline 23.00 & 1.00 & 5.01 & 2.50 & 1.67 & 1.25 & 1.17 & 1.24 & 0.99 & 1.03 & 1.30 & 1.10 \\
\hline 24.00 & 1.00 & 5.01 & 2.50 & 1.67 & 1.25 & 1.17 & 1.21 & 0.96 & 1.00 & 1.29 & 1.10 \\
\hline 25.00 & 1.00 & 5.01 & 2.50 & 1.67 & 1.25 & 1.17 & 1.19 & 0.93 & 0.97 & 1.27 & 1.10 \\
\hline 26.00 & 1.00 & 5.01 & 2.50 & 1.67 & 1.25 & 1.17 & 1.17 & 0.90 & 0.95 & 1.26 & 1.10 \\
\hline 27.00 & 1.00 & 5.01 & 2.50 & 1.67 & 1.25 & 1.17 & 1.15 & 0.88 & 0.92 & 1.24 & 1.10 \\
\hline 28.00 & 1.00 & 5.01 & 2.50 & 1.67 & 1.25 & 1.16 & 1.13 & 0.85 & 0.90 & 1.23 & 1.10 \\
\hline 29.00 & 1.00 & 5.01 & 2.50 & 1.67 & 1.25 & 1.16 & 1.11 & 0.83 & 0.88 & 1.22 & 1.10 \\
\hline 30.00 & 1.00 & 5.01 & 2.50 & 1.67 & 1.25 & 1.16 & 1.09 & 0.81 & 0.85 & 1.20 & 1.10 \\
\hline
\end{tabular}


Table 3.12: Efficiency for different values of $\Delta^{2}$ at $p=10$ and $k=d=0.1,0.5$ and 0.9

\begin{tabular}{|c|c|c|c|c|c|c|c|c|c|c|c|c|c|}
\hline \multirow[t]{2}{*}{$\Delta^{2}$} & \multirow[t]{2}{*}{ LSE } & \multicolumn{6}{|c|}{ LASSO } & \multicolumn{3}{|c|}{ Ridge } & \multicolumn{3}{|c|}{ Liu } \\
\hline & & $q=1$ & $q=3$ & $q=5$ & $q=7$ & $q=9$ & $q=10$ & $k=0.1$ & $k=0.5$ & $k=0.9$ & $d=0.1$ & $d=0.5$ & $\mathrm{~d}=0.9$ \\
\hline 0.00 & 1.00 & 8.92 & 4.46 & 2.97 & 2.23 & 1.78 & 1.62 & 1.21 & 2.25 & 3.61 & 3.31 & 1.78 & 1.11 \\
\hline 1.00 & 1.00 & 8.92 & 4.46 & 2.97 & 2.23 & 1.78 & 1.62 & 1.21 & 2.20 & 3.34 & 3.10 & 1.76 & 1.11 \\
\hline 2.00 & 1.00 & 8.92 & 4.46 & 2.97 & 2.23 & 1.78 & 1.62 & 1.21 & 2.14 & 3.11 & 2.92 & 1.74 & 1.11 \\
\hline 3.00 & 1.00 & 8.92 & 4.46 & 2.97 & 2.23 & 1.78 & 1.62 & 1.21 & 2.09 & 2.90 & 2.75 & 1.72 & 1.11 \\
\hline 4.00 & 1.00 & 8.92 & 4.46 & 2.97 & 2.23 & 1.78 & 1.62 & 1.21 & 2.05 & 2.73 & 2.61 & 1.70 & 1.11 \\
\hline 5.00 & 1.00 & 8.92 & 4.46 & 2.97 & 2.23 & 1.78 & 1.62 & 1.20 & 2.00 & 2.57 & 2.48 & 1.68 & 1.11 \\
\hline 6.00 & 1.00 & 8.92 & 4.46 & 2.97 & 2.23 & 1.78 & 1.62 & 1.20 & 1.96 & 2.43 & 2.36 & 1.67 & 1.11 \\
\hline 7.00 & 1.00 & 8.92 & 4.46 & 2.97 & 2.23 & 1.78 & 1.62 & 1.20 & 1.91 & 2.30 & 2.25 & 1.65 & 1.11 \\
\hline 8.00 & 1.00 & 8.92 & 4.46 & 2.97 & 2.23 & 1.78 & 1.62 & 1.20 & 1.88 & 2.19 & 2.15 & 1.63 & 1.11 \\
\hline 9.00 & 1.00 & 8.92 & 4.46 & 2.97 & 2.23 & 1.78 & 1.62 & 1.20 & 1.84 & 2.09 & 2.06 & 1.62 & 1.11 \\
\hline 10.00 & 1.00 & 8.92 & 4.46 & 2.97 & 2.23 & 1.78 & 1.62 & 1.20 & 1.80 & 1.99 & 1.98 & 1.60 & 1.10 \\
\hline 11.00 & 1.00 & 8.92 & 4.46 & 2.97 & 2.23 & 1.78 & 1.62 & 1.20 & 1.76 & 1.91 & 1.90 & 1.58 & 1.10 \\
\hline 12.00 & 1.00 & 8.92 & 4.46 & 2.97 & 2.23 & 1.78 & 1.62 & 1.20 & 1.73 & 1.83 & 1.83 & 1.57 & 1.10 \\
\hline 13.00 & 1.00 & 8.92 & 4.46 & 2.97 & 2.23 & 1.78 & 1.62 & 1.19 & 1.70 & 1.76 & 1.77 & 1.55 & 1.10 \\
\hline 14.00 & 1.00 & 8.92 & 4.46 & 2.97 & 2.23 & 1.78 & 1.62 & 1.19 & 1.67 & 1.69 & 1.71 & 1.54 & 1.10 \\
\hline 15.00 & 1.00 & 8.92 & 4.46 & 2.97 & 2.23 & 1.78 & 1.62 & 1.19 & 1.64 & 1.63 & 1.65 & 1.52 & 1.10 \\
\hline 16.00 & 1.00 & 8.92 & 4.46 & 2.97 & 2.23 & 1.78 & 1.62 & 1.19 & 1.61 & 1.57 & 1.60 & 1.51 & 1.10 \\
\hline 17.00 & 1.00 & 8.92 & 4.46 & 2.97 & 2.23 & 1.78 & 1.62 & 1.19 & 1.58 & 1.52 & 1.55 & 1.50 & 1.10 \\
\hline 18.00 & 1.00 & 8.92 & 4.46 & 2.97 & 2.23 & 1.78 & 1.62 & 1.19 & 1.55 & 1.47 & 1.50 & 1.48 & 1.10 \\
\hline 19.00 & 1.00 & 8.92 & 4.46 & 2.97 & 2.23 & 1.78 & 1.62 & 1.19 & 1.53 & 1.42 & 1.46 & 1.47 & 1.10 \\
\hline 20.00 & 1.00 & 8.92 & 4.46 & 2.97 & 2.23 & 1.78 & 1.62 & 1.19 & 1.50 & 1.38 & 1.41 & 1.45 & 1.10 \\
\hline 21.00 & 1.00 & 8.92 & 4.46 & 2.97 & 2.23 & 1.78 & 1.62 & 1.19 & 1.48 & 1.34 & 1.37 & 1.44 & 1.10 \\
\hline 22.00 & 1.00 & 8.92 & 4.46 & 2.97 & 2.23 & 1.78 & 1.62 & 1.18 & 1.45 & 1.30 & 1.34 & 1.43 & 1.10 \\
\hline 23.00 & 1.00 & 8.92 & 4.46 & 2.97 & 2.23 & 1.78 & 1.62 & 1.18 & 1.43 & 1.26 & 1.30 & 1.42 & 1.10 \\
\hline 24.00 & 1.00 & 8.92 & 4.46 & 2.97 & 2.23 & 1.78 & 1.62 & 1.18 & 1.41 & 1.23 & 1.27 & 1.40 & 1.10 \\
\hline 25.00 & 1.00 & 8.92 & 4.46 & 2.97 & 2.23 & 1.78 & 1.62 & 1.18 & 1.38 & 1.19 & 1.24 & 1.39 & 1.10 \\
\hline 26.00 & 1.00 & 8.92 & 4.46 & 2.97 & 2.23 & 1.78 & 1.62 & 1.18 & 1.36 & 1.16 & 1.21 & 1.38 & 1.10 \\
\hline 27.00 & 1.00 & 8.92 & 4.46 & 2.97 & 2.23 & 1.78 & 1.62 & 1.18 & 1.34 & 1.13 & 1.18 & 1.37 & 1.10 \\
\hline 28.00 & 1.00 & 8.92 & 4.46 & 2.97 & 2.23 & 1.78 & 1.62 & 1.18 & 1.32 & 1.10 & 1.15 & 1.36 & 1.10 \\
\hline 29.00 & 1.00 & 8.92 & 4.46 & 2.97 & 2.23 & 1.78 & 1.62 & 1.18 & 1.30 & 1.08 & 1.12 & 1.34 & 1.10 \\
\hline 30.00 & 1.00 & 8.92 & 4.46 & 2.97 & 2.23 & 1.78 & 1.62 & 1.17 & 1.29 & 1.05 & 1.10 & 1.33 & 1.10 \\
\hline
\end{tabular}




\section{COMPARISON OF RIDGE, LIU AND TWO PARAMETER BIASED ESTIMATORS}

Since the comparison of Ridge, Liu and Two parameter biased estimator is limited in literature, in this chapter, I review some estimators for estimating ridge parameter $k$ and optimum value of shrinkage parameter $d$. Since a theoretical comparison is not possible, I will do a simulation study to compare the performance of the estimators in the sense of smaller MSE.

\subsection{Ridge, Liu and Two parameter biased Estimators.}

Using the canonical form of linear model, we know from (2.4) that the MSE of generalized ridge regression estimator is,

$$
\operatorname{MSE}\left(\hat{\alpha}_{k}\right)=\sigma^{2} \sum_{i=1}^{p} \frac{\lambda_{i}}{\left(\lambda_{i}+k_{i}\right)^{2}}+\sum_{i=1}^{p} \frac{k_{i}^{2} \lambda_{i}^{2}}{\left(\lambda_{i}+k_{i}\right)^{2}}
$$

Note that in the previous Chapter 3, I compare the estimators based on the orthonormal regression model because of LASSO estimator, as the risk function is only available in orthonormal form.

It follows from Hoerl and Kennard (1970) that the value of $k_{i}$ which minimizes the $\operatorname{MSE}\left(\hat{\alpha}_{k}\right)$ is

$$
k_{i}=\frac{\sigma^{2}}{\alpha_{i}^{2}}
$$

where $\sigma^{2}$ represents the error variance of the model and $\alpha_{i}$ is the $i$ th element of $\alpha$. Hoerl and Kennard (1970), suggested to replace $\sigma^{2}$ and $\alpha_{i}^{2}$ by their corresponding unbiased estimators. That is,

$$
\hat{k}_{i}=\frac{\widehat{\sigma}^{2}}{\widehat{\alpha}_{i}^{2}}
$$


Now I will review some estimators, they are as follows:

1. Estimator based on Hoerl, Kennard and Baldwin (1975) (thereafter $\hat{k}_{H K B}$ or HKB), proposed an estimator of $k$ by taking harmonic mean of $\hat{k}_{i}$ in (4.1).

$$
\hat{k}_{H K B}=\frac{p \widehat{\sigma}^{2}}{\widehat{\alpha}^{\prime} \widehat{\alpha}} .
$$

2. Estimator based on Lawless and Wang (1976) (thereafter $\hat{k}_{L W}$ or LW), proposed the following estimator:

$$
\hat{k}_{L W}=\frac{p \widehat{\sigma}^{2}}{\widehat{\alpha}^{\prime} X \prime X \widehat{\alpha}}
$$

3. Kibria (2003) proposed an estimator by taking the geometric mean of $\hat{k}_{i}$, which produced the following estimator:

$$
\hat{k}_{G M}=\frac{\widehat{\sigma}^{2}}{\left(\prod_{i=1}^{p} \widehat{\alpha}_{i}^{2}\right)^{\frac{1}{p}}} .
$$

4. Muniz and Kibria (2009) proposed estimators by taking geometric mean and square root of estimator proposed by Alkhamisi and Shukur (2006). Suppose $m_{i}=\sqrt{\sqrt{\widehat{\sigma}^{2}}}$, then following estimators were proposed:

$$
\begin{aligned}
\hat{k}_{K M 4} & =\left(\prod_{i=1}^{p} \frac{1}{m_{i}}\right)^{\frac{1}{p}} . \\
\text { and } \quad \hat{k}_{K M 5} & =\left(\prod_{i=1}^{p} m_{i}\right)^{\frac{1}{p}} .
\end{aligned}
$$

5. Estimator based on Alkhamisi and Shukur (2006) (thereafter $\hat{k}_{A S}$ or AS), proposed the following estimator:

$$
\hat{k}_{A S}=\left(\frac{\widehat{\sigma}^{2}}{\max \left(\hat{\alpha}_{i}^{2}\right)}+\frac{1}{\lambda_{i}}\right), i=1,2, \ldots, p
$$

These are the few among many estimators suggested by researchers that will be compared in the study. For more in the estimation of $k$, I refer our readers to Kibria (2003), Khalaf and Shukur (2005), Muniz and Kibria (2009), Alkhamisi and Shukur 
(2006), Khalaf (2012), Aslam (2014), Dorugade (2013) and very recently Kibria and Banik (2015) among others.

From (2.6), we know the MSE of Liu estimator in canonical form is given by,

$$
\operatorname{MSE}\left(\hat{\alpha}_{d}\right)=\sigma^{2} \sum_{i=1}^{p} \frac{\left(\lambda_{i}+d\right)^{2}}{\lambda_{i}\left(\lambda_{i}+1\right)^{2}}+(d-1)^{2} \sum_{i=1}^{p} \frac{\alpha_{i}^{2}}{\left(\lambda_{i}+1\right)^{2}}
$$

Liu (1993) suggested that the $\operatorname{MSE}\left(\hat{\alpha}_{d}\right)$ is minimized at

$$
d_{i}=\frac{\alpha_{i}^{2}-\sigma^{2}}{\alpha_{i}^{2}+\left(\sigma^{2} / \lambda_{i}\right)}, \quad i=1,2, \ldots, p
$$

Now, considering the canonical form in (2.1), the estimate for two parameter biased estimator is obtained as,

$$
\hat{\alpha}_{k, d}=(\boldsymbol{\Lambda}+k \mathbf{I})^{-1}\left(\mathbf{Q}^{\prime} \mathrm{y}-d \hat{\alpha}\right) .
$$

The relationship between linear regression model and orthogonal model is as,

$\hat{\beta}_{k, d}=\mathbf{Q} \hat{\alpha}_{k, d} \cdot \operatorname{MSE}\left(\hat{\alpha}_{k, d}\right)$ is obtained as,

$$
\operatorname{MSE}\left(\hat{\alpha}_{k, d}\right)=\sigma^{2} \sum_{i=1}^{p} \frac{\left(d-\lambda_{i}\right)^{2}}{\lambda_{i}\left(\lambda_{i}+k\right)^{2}}+\sum_{i=1}^{p} \frac{(d+k)^{2} \alpha_{i}^{2}}{\left(\lambda_{i}+k\right)^{2}}
$$

It can be shown that $(4.10)$ is minimized at

$$
d_{o p t}=\frac{\sum_{i=1}^{p} \frac{\sigma^{2}-k \alpha_{i}^{2}}{\left(\lambda_{i}+k\right)^{2}}}{\sum_{i=1}^{p} \frac{\left(\lambda_{i} \alpha_{i}^{2}+\sigma^{2}\right)}{\lambda_{i}\left(\lambda_{i}+k\right)^{2}}} .
$$

As mentioned earlier that the two parameter biased estimator has less MSE than ridge regression estimator, also it allows larger values of $k$ and thus can fully address the problem of ill conditioning. The understanding of the superior performance of two parameter biased estimator over ridge regression estimator can be theoretically 
explained as follows, we know that adding a value of $k$ deals with ill conditioning of $\mathbf{X}^{\prime} \mathbf{X}$ in the model but practically ridge regression does not allow a very large value of $k$ as it creates a bias. Because of this bias the problem of ill conditioning is not fully addressed. In the two parameter biased estimator of $\hat{\beta}_{k, d}, k$ can be used exclusively to control the illconditioning of $\mathbf{X}^{\prime} \mathbf{X}+k \mathbf{l}$, inevitably some bias is generated and hence the second parameter $d$ is used to improve the fit . I choose the ridge regression estimators discussed earlier from equation (4.2) - (4.7). After the $k$ is selected, we can use $d_{\text {opt }}$ to choose $d$ from (4.11). Thus the two parameters in $\hat{\beta}_{k, d}$ are selected.

\subsection{Monte Carlo Simulation.}

In this section, I want to use a simulation study to illustrate the behavior of all the estimators discussed in section 4.1. The simulation is carried out under different degrees of multicollinearity, following McDonald and Galarneau (1975) which was also adopted by Gibbons (1981) and Kibria (2003).The explanatory variables were generated using the following equation:

$$
x_{i j}=\left(1-\gamma^{2}\right)^{\frac{1}{2}} z_{i j}+\gamma z_{i p}, i=1,2, \ldots, \mathrm{n} ; j=1,2, \ldots, \mathrm{p}
$$

where $z_{i j}$ are independent standard normal pseudo-random numbers, and $\gamma^{2}$ is the theoretical correlation between any two explanatory variables. These variables are standardized so that $\mathbf{X}^{\prime} \mathbf{X}$ and $\mathbf{X}^{\prime} \mathbf{y}$ are in correlation forms. The $n$ observations for the dependent variable are determined by,

$$
y_{i}=\beta_{1} x_{i 1}+\beta_{2} x_{i 2}+\cdots+\beta_{p} x_{i p}+\varepsilon_{i}, i=1,2, \ldots, n,
$$

where $\varepsilon_{i}$ are independent normal pseudo-random numbers with mean 0 and variance $\sigma^{2}$ 
Since my primary interest lies in the performance of our proposed estimators according to strength of multicollinearity, I considered three sets of correlation corresponding to $\gamma=$ $0.7,0.8,0.9$. I also want to see the effect of the sample size on the number of regressors so I vary sample size between 15 and 50 , and explanatory variables between 4 and 10 . I investigate five values of sigma $\sigma: 0.1,0.5,1,4,10$; or equivalently, five signal-to-noise ratios: $100,4,1,0.0625,0.01$. For each set of explanatory variables, I follow Newhouse and Oman (1971) conclusion for choosing the coefficient vector to minimize the MSE. When the MSE is function of $\beta, \sigma^{2}$ and $k$ and explanatory variables are fixed, they suggested to choose the coefficient vector corresponding to the largest eigen value of $\mathbf{X}^{\prime} \mathbf{X}$ matrix subject to constraint $\beta^{\prime} \beta=1$. One can also use the coefficient vector corresponding to the smallest eigen value but the results about performance of estimators do not differ significantly. The eigen values and the regression coefficients of $\mathbf{X}^{\prime} \mathbf{X}$ for different set on $n, p, \gamma$ and $\rho^{2}$ are given in Table 4.1 .

For the given values of $n, p, \beta, \lambda, \gamma$ and $\rho^{2}$, the set of explanatory variables are generated. Then the experiment was repeated 2000 times by generating new error terms in (4.13). Then the values of ridge parameters $k$ of the different estimators, $d$ for Liu estimator and optimum $d s$ for two parameter estimators and their corresponding estimators as well as average MSEs were estimated. The MSEs for the estimators are calculated as follows

$$
\operatorname{MSE}(\hat{\alpha})=\frac{1}{2000} \sum_{r=1}^{2000}\left(\hat{\alpha}_{(r)}-\alpha\right)^{\prime}\left(\hat{\alpha}_{(r)}-\alpha\right)
$$

In this simulation study, twelve estimators are compared and their simulated MSE are presented in Tables 4.2-4.13 respectively. For a more in depth idea about which estimator performs uniformly better than LSE can be obtained from Tables $4.14-4.16$. Along with MSEs, average values of $k$, standard deviation of $k$ and the percentage for 
which the given estimator out performs LSE are provided. The twelve estimators compared are:

1. LSE: Least square estimator.

2. HKB: Ridge regression with $\hat{k}_{H K B}$

3. LW: Ridge regression with $\hat{k}_{L W}$

4. GM: Ridge regression with $\hat{k}_{G M}$

5. KM4: Ridge regression with $\hat{k}_{K M 4}$

6. KM5: Ridge regression with $\hat{k}_{K M 5}$

7. AS: Ridge regression with $\hat{k}_{A S}$

8. TPHKB: Two parameter biased estimator with $\hat{k}_{H K B}, \hat{\beta}=\hat{\beta}_{L S}$ and $d_{\text {opt }}$

9. TPGM: Two parameter biased estimator with $\hat{k}_{G M}$ and $\hat{\beta}=\hat{\beta}_{L S}$ and $d_{o p t}$

10. TPKM5: Two parameter biased estimator with $\hat{k}_{K M 5}$ and $\hat{\beta}=\hat{\beta}_{L S}$ and $d_{o p t}$

11. TPAS: Two parameter biased estimator with $\hat{k}_{A S}$ and $\hat{\beta}=\hat{\beta}_{L S}$ and $d_{\text {opt }}$

12. Liu: Liu estimator with optimum $d$ 
Table 4.1: Values of $\lambda$ and $\beta$ used in simulation for $n=50$ and different $p$

\begin{tabular}{|c|c|c|c|c|c|c|}
\hline \multicolumn{7}{|l|}{$Y$} \\
\hline & 0.7 & 0.8 & 0.9 & 0.7 & 0.8 & 0.9 \\
\hline$n$ & \multicolumn{3}{|c|}{$n=50, p=4$} & \multicolumn{3}{|c|}{$n=50, p=10$} \\
\hline$\lambda_{1}$ & 408.24 & 378.011 & 338.597 & 295.141 & 344.529 & 395.777 \\
\hline$\lambda_{2}$ & 27.3165 & 19.2807 & 10.1746 & 48.5102 & 34.2557 & 18.0848 \\
\hline$\lambda_{3}$ & 21.0514 & 14.8643 & 7.84855 & 39.0282 & 27.749 & 14.7464 \\
\hline$\lambda_{4}$ & 10.1791 & 6.69967 & 3.13001 & 31.5056 & 22.1267 & 11.6163 \\
\hline$\lambda_{5}$ & & & & 25.2457 & 17.8418 & 9.42814 \\
\hline$\lambda_{6}$ & & & & 21.1363 & 14.8799 & 7.83493 \\
\hline$\lambda_{7}$ & & & & 19.5329 & 13.8011 & 7.29219 \\
\hline$\lambda_{8}$ & & & & 17.3463 & 12.154 & 6.37162 \\
\hline$\lambda_{9}$ & & & & 10.5365 & 7.23757 & 3.76573 \\
\hline$\lambda_{10}$ & & & & 8.04895 & 4.9286 & 2.10146 \\
\hline$\beta_{1}$ & -0.3897 & -0.405 & -0.4286 & -0.271 & -0.2849 & -0.2977 \\
\hline$\beta_{2}$ & -0.4015 & -0.4152 & -0.4365 & -0.2063 & -0.2359 & -0.2655 \\
\hline$\beta_{3}$ & -0.4353 & -0.4442 & -0.4582 & -0.2904 & -0.3002 & -0.3085 \\
\hline$\beta_{4}$ & -0.7053 & -0.6828 & -0.6448 & -0.3923 & -0.3693 & -0.3485 \\
\hline$\beta_{5}$ & & & & -0.2645 & -0.2765 & -0.2896 \\
\hline$\beta_{6}$ & & & & -0.271 & -0.282 & -0.2938 \\
\hline$\beta_{7}$ & & & & -0.3012 & -0.3051 & -0.3093 \\
\hline$\beta_{8}$ & & & & -0.2845 & -0.2893 & -0.2966 \\
\hline$\beta_{9}$ & & & & -0.2545 & -0.2674 & -0.2828 \\
\hline$\beta_{10}$ & & & & -0.5157 & -0.4822 & -0.4365 \\
\hline
\end{tabular}


Table 4.2: Estimated MSE with $n=15, p=4, y=0.7$

\begin{tabular}{|c|c|c|c|c|c|c|c|c|c|c|c|c|}
\hline sigma & LS & HKB & LW & GM & KM4 & KM5 & AS & TPHKB & TPGM & TPKM5 & TPAS & LIU \\
\hline 0.1 & 0.0099914 & 0.0096636 & 0.0099753 & 0.0131883 & 0.0071161 & 0.006417 & 0.0073855 & 0.0051448 & 0.0062297 & 0.0056696 & 0.0051374 & 0.0036321 \\
\hline 0.5 & 0.2497838 & 0.1579487 & 0.2376406 & 0.1505154 & 0.1872188 & 0.1316426 & 0.1687266 & 0.1168612 & 0.1206345 & 0.1145756 & 0.1205216 & 0.0913687 \\
\hline 1 & 0.9991353 & 0.4852051 & 0.8653898 & 0.5663049 & 0.7727402 & 0.50718 & 0.5704635 & 0.4424265 & 0.4480063 & 0.4406052 & 0.4593035 & 0.3576757 \\
\hline 4 & 15.986164 & 7.429741 & 11.907569 & 10.309655 & 13.109458 & 8.333695 & 9.040674 & 6.969378 & 6.895514 & 7.000231 & 7.054533 & 5.745691 \\
\hline 10 & 99.91353 & 45.80064 & 72.06577 & 69.54592 & 84.51883 & 52.99509 & 58.57629 & 42.65201 & 42.06875 & 42.7154 & 43.05988 & 34.62554 \\
\hline
\end{tabular}

Table 4.3: Estimated MSE with $n=15, p=4, y=0.8$

\begin{tabular}{|c|c|c|c|c|c|c|c|c|c|c|c|c|}
\hline sigma & LS & HKB & LW & GM & KM4 & KM5 & AS & TPHKB & TPGM & TPKM5 & TPAS & LIU \\
\hline 0.1 & 0.0149578 & 0.0142023 & 0.0149196 & 0.0117327 & 0.0098214 & 0.0084132 & 0.0095694 & 0.0072895 & 0.0079226 & 0.0073661 & 0.0074577 & 0.0053886 \\
\hline 0.5 & 0.3739443 & 0.2128902 & 0.3441016 & 0.2152643 & 0.2550967 & 0.1972852 & 0.2285522 & 0.1705009 & 0.168675 & 0.1652844 & 0.1803241 & 0.1346386 \\
\hline 1 & 1.4957771 & 0.7116152 & 1.2111367 & 0.8746838 & 1.0431655 & 0.7869669 & 0.8420659 & 0.6617411 & 0.6591206 & 0.6576636 & 0.696571 & 0.5408818 \\
\hline 4 & 23.932433 & 11.426643 & 17.242251 & 16.984002 & 17.895037 & 13.749235 & 15.085919 & 10.529856 & 10.393935 & 10.511884 & 10.797726 & 8.642682 \\
\hline 10 & 149.57771 & 69.78893 & 106.47177 & 112.01861 & 115.43902 & 87.36506 & 95.34936 & 64.26612 & 63.45536 & 63.9611 & 65.93505 & 52.79462 \\
\hline
\end{tabular}

Table 4.4: Estimated MSE with $n=15, p=4, y=0.9$

\begin{tabular}{|c|c|c|c|c|c|c|c|c|c|c|c|c|}
\hline sigma & LS & HKB & LW & GM & KM4 & KM5 & AS & TPHKB & TPGM & TPKM5 & TPAS & LIU \\
\hline 0.1 & 0.0308692 & 0.0276922 & 0.030685 & 0.0174065 & 0.0201844 & 0.0161961 & 0.0209403 & 0.0146239 & 0.0140715 & 0.0135773 & 0.0161779 & 0.0109332 \\
\hline 0.5 & 0.7717296 & 0.3795884 & 0.6431317 & 0.4331345 & 0.4877646 & 0.4228886 & 0.5235205 & 0.3367361 & 0.3270864 & 0.3262764 & 0.387198 & 0.2712389 \\
\hline 1 & 3.086918 & 1.393182 & 2.255883 & 1.83192 & 1.924166 & 1.727677 & 2.066281 & 1.287673 & 1.270758 & 1.274197 & 1.449438 & 1.067342 \\
\hline 4 & 49.39069 & 22.48665 & 33.66357 & 34.98506 & 31.08769 & 30.24301 & 35.08837 & 20.41577 & 20.17477 & 20.22026 & 22.26608 & 16.94436 \\
\hline 10 & 308.6918 & 145.0339 & 208.3476 & 247.3803 & 204.9827 & 208.3298 & 233.1699 & 130.9143 & 129.0636 & 129.3599 & 143.0726 & 108.503 \\
\hline
\end{tabular}


Table 4.5: Estimated MSE with $n=15, p=10, y=0.7$

\begin{tabular}{|c|c|c|c|c|c|c|c|c|c|c|c|c|}
\hline sigma & LS & HKB & LW & GM & KM4 & KM5 & AS & TPHKB & TPGM & TPKM5 & TPAS & LIU \\
\hline 0.1 & 0.046798 & 0.0367564 & 0.0449406 & 0.0504945 & 0.0301879 & 0.0341312 & 0.0357979 & 0.0250479 & 0.0254408 & 0.0225386 & 0.029043 & 0.0169642 \\
\hline 0.5 & 1.169951 & 0.6419494 & 0.8767881 & 0.9961206 & 0.7611975 & 0.8354619 & 0.8682475 & 0.5528522 & 0.5351144 & 0.5354022 & 0.6850901 & 0.4183946 \\
\hline 1 & 4.679804 & 2.634919 & 3.274944 & 3.893886 & 3.04824 & 3.292386 & 3.352814 & 2.158172 & 2.092785 & 2.120595 & 2.606198 & 1.659148 \\
\hline 4 & 74.87686 & 41.9769 & 50.30539 & 62.59726 & 49.30746 & 52.40536 & 52.35224 & 33.63644 & 32.75617 & 33.1739 & 38.8979 & 25.91715 \\
\hline 10 & 467.9804 & 275.8618 & 317.3916 & 421.0058 & 317.7537 & 353.4829 & 352.3179 & 216.6397 & 209.405 & 212.5535 & 252.7841 & 166.5883 \\
\hline
\end{tabular}

Table 4.6: Estimated MSE with $n=15, p=10, y=0.8$

\begin{tabular}{|c|c|c|c|c|c|c|c|c|c|c|c|c|}
\hline Sigma & LS & HKB & LW & GM & KM4 & KM5 & AS & TPHKB & TPGM & TPKM5 & TPAS & LIU \\
\hline 0.1 & 0.0719235 & 0.0511077 & 0.0671433 & 0.0651366 & 0.0456673 & 0.0538282 & 0.0586689 & 0.0372895 & 0.034643 & 0.0329088 & 0.0474027 & 0.0255868 \\
\hline 0.5 & 1.7980877 & 1.0060598 & 1.2464344 & 1.51001 & 1.1214231 & 1.3375363 & 1.4361408 & 0.8303941 & 0.7946134 & 0.8015447 & 1.1409943 & 0.6363006 \\
\hline 1 & 7.192351 & 4.222495 & 4.709176 & 6.283716 & 4.585819 & 5.556869 & 5.886123 & 3.305163 & 3.189974 & 3.225929 & 4.460466 & 2.565731 \\
\hline 4 & 115.07761 & 70.12109 & 74.91071 & 106.35673 & 74.02306 & 93.24769 & 97.48011 & 53.47523 & 51.6721 & 52.20775 & 69.87783 & 41.88652 \\
\hline 10 & 719.2351 & 436.202 & 465.3851 & 679.7789 & 467.2957 & 595.0823 & 615.6397 & 331.7632 & 320.5374 & 323.4525 & 435.8849 & 258.3578 \\
\hline
\end{tabular}

Table 4.7: Estimated MSE with $n=15, p=10, \gamma=0.9$

\begin{tabular}{|c|c|c|c|c|c|c|c|c|c|c|c|c|}
\hline Sigma & LS & HKB & LW & GM & KM4 & KM5 & AS & TPHKB & TPGM & TPKM5 & TPAS & LIU \\
\hline 0.1 & 0.1529433 & 0.0918929 & 0.1307873 & 0.1276389 & 0.110417 & 0.1207058 & 0.1364077 & 0.0757656 & 0.0670437 & 0.0665439 & 0.1138187 & 0.0538967 \\
\hline 0.5 & 3.823582 & 2.249317 & 2.447658 & 3.359379 & 2.770679 & 3.166831 & 3.505374 & 1.73034 & 1.667656 & 1.673758 & 2.851258 & 1.364865 \\
\hline 1 & 15.294327 & 9.135178 & 9.477046 & 13.425396 & 10.804042 & 12.582917 & 13.777292 & 6.884303 & 6.649564 & 6.679168 & 10.921922 & 5.484152 \\
\hline 4 & 244.70923 & 150.31716 & 151.87863 & 229.57841 & 174.72612 & 212.79638 & 229.73494 & 111.2674 & 107.79617 & 108.12833 & 175.16839 & 88.38628 \\
\hline 10 & 1529.4327 & 948.2393 & 947.8756 & 1476.0187 & 1086.8153 & 1363.6703 & 1456.1289 & 699.2951 & 677.4846 & 678.6354 & 1108.8051 & 554.5174 \\
\hline
\end{tabular}


Table 4.8: Estimated MSE with $n=50, p=4, y=0.7$

\begin{tabular}{|c|c|c|c|c|c|c|c|c|c|c|c|c|}
\hline Sigma & LS & HKB & LW & GM & KM4 & KM5 & AS & TPHKB & TPGM & TPKM5 & TPAS & LIU \\
\hline 0.1 & 0.0020854 & 0.0020716 & 0.002085 & 0.0111715 & 0.0019603 & 0.0019372 & 0.0020471 & 0.0016525 & 0.0018626 & 0.0017617 & 0.0016391 & 0.0007601 \\
\hline 0.5 & 0.0521353 & 0.0451748 & 0.0518841 & 0.0529514 & 0.0498268 & 0.0344652 & 0.0492616 & 0.0267332 & 0.0324605 & 0.0273893 & 0.0267306 & 0.0190356 \\
\hline 1 & 0.2085411 & 0.1402165 & 0.204994 & 0.1448037 & 0.200697 & 0.1289923 & 0.1770852 & 0.1001898 & 0.1113137 & 0.0999975 & 0.1018641 & 0.0763115 \\
\hline 4 & 3.336658 & 1.528862 & 3.041341 & 1.927381 & 3.247668 & 1.854959 & 1.756108 & 1.45235 & 1.470178 & 1.491496 & 1.470347 & 1.192816 \\
\hline 10 & 20.85411 & 9.373097 & 18.168696 & 13.010668 & 20.426873 & 10.952904 & 11.361576 & 8.850362 & 8.754806 & 9.111642 & 8.877008 & 7.168491 \\
\hline
\end{tabular}

Table 4.9: Estimated MSE with $n=50, p=4, y=0.8$

\begin{tabular}{|c|c|c|c|c|c|c|c|c|c|c|c|c|}
\hline sigma & LS & HKB & LW & GM & KM4 & KM5 & AS & TPHKB & TPGM & TPKM5 & TPAS & LIU \\
\hline 0.1 & 0.0030391 & 0.0030086 & 0.0030381 & 0.0075621 & 0.0027307 & 0.0023205 & 0.0029178 & 0.0019287 & 0.0023604 & 0.0021346 & 0.0019158 & 0.0011038 \\
\hline 0.5 & 0.0759784 & 0.0620279 & 0.0753433 & 0.0579413 & 0.0703267 & 0.0461828 & 0.0690433 & 0.0371801 & 0.0415205 & 0.0367882 & 0.0375378 & 0.0277636 \\
\hline 1 & 0.3039136 & 0.1844956 & 0.2951541 & 0.1796017 & 0.2846096 & 0.1744956 & 0.2390452 & 0.1401776 & 0.1456594 & 0.138994 & 0.1443675 & 0.1092562 \\
\hline 4 & 4.862617 & 2.232179 & 4.281019 & 2.861068 & 4.639933 & 2.57811 & 2.578353 & 2.106984 & 2.105738 & 2.161598 & 2.131829 & 1.740549 \\
\hline 10 & 30.39136 & 13.85273 & 25.79729 & 19.99025 & 29.2936 & 15.71448 & 17.0922 & 12.997 & 12.87357 & 13.27169 & 13.0375 & 10.6188 \\
\hline
\end{tabular}

Table 4.10: Estimated MSE with $n=50, p=4, \gamma=0.9$

\begin{tabular}{|c|c|c|c|c|c|c|c|c|c|c|c|c|}
\hline sigma & LS & HKB & LW & GM & KM4 & KM5 & AS & TPHKB & TPGM & TPKM5 & TPAS & LIU \\
\hline 0.1 & 0.0060537 & 0.0059273 & 0.0060492 & 0.0061443 & 0.0047564 & 0.0037309 & 0.0051914 & 0.0030589 & 0.003498 & 0.0031532 & 0.0030656 & 0.0021582 \\
\hline 0.5 & 0.1513422 & 0.1066449 & 0.1481297 & 0.087148 & 0.1261314 & 0.0818556 & 0.118864 & 0.0701819 & 0.069795 & 0.0675112 & 0.0720618 & 0.0537323 \\
\hline 1 & 0.6053686 & 0.3128591 & 0.5648157 & 0.3342905 & 0.5182376 & 0.3166532 & 0.3927557 & 0.2672132 & 0.2641652 & 0.2647211 & 0.2797057 & 0.2121683 \\
\hline 4 & 9.685898 & 4.401896 & 7.982438 & 5.807901 & 8.623953 & 4.845213 & 5.203612 & 4.108073 & 4.07844 & 4.166959 & 4.139735 & 3.400016 \\
\hline 10 & 60.53686 & 27.35599 & 48.81411 & 41.26191 & 55.1935 & 30.91536 & 35.1995 & 25.35054 & 25.10372 & 25.55998 & 25.6013 & 20.87807 \\
\hline
\end{tabular}


Table 4.11: Estimated MSE with $n=50, p=10, \gamma=0.7$

\begin{tabular}{|c|c|c|c|c|c|c|c|c|c|c|c|c|}
\hline sigma & LS & HKB & LW & GM & KM4 & KM5 & AS & TPHKB & TPGM & TPKM5 & TPAS & LIU \\
\hline 0.1 & 0.0057045 & 0.0055999 & 0.0057021 & 0.0504328 & 0.0055077 & 0.005087 & 0.0055631 & 0.0039923 & 0.0052433 & 0.0044939 & 0.0039707 & 0.0020024 \\
\hline 0.5 & 0.1426117 & 0.1051151 & 0.1412005 & 0.1551477 & 0.1383003 & 0.0845454 & 0.1333832 & 0.0780738 & 0.0896033 & 0.0766101 & 0.0801394 & 0.0501204 \\
\hline 1 & 0.5704467 & 0.327528 & 0.5517138 & 0.4475692 & 0.5543025 & 0.3317808 & 0.4764835 & 0.2974342 & 0.3065563 & 0.2969289 & 0.3157866 & 0.2021973 \\
\hline 4 & 9.127148 & 4.638433 & 8.1892 & 6.174592 & 8.903293 & 5.092452 & 5.185746 & 4.416422 & 4.305396 & 4.578231 & 4.600128 & 3.186709 \\
\hline 10 & 57.04467 & 29.22233 & 50.32267 & 39.98599 & 55.77135 & 31.54804 & 32.59912 & 27.56371 & 26.66685 & 28.53667 & 28.55483 & 19.76678 \\
\hline
\end{tabular}

Table 4.12: Estimated MSE with $n=50, p=10, y=0.8$

\begin{tabular}{|c|c|c|c|c|c|c|c|c|c|c|c|c|}
\hline sigma & LS & HKB & LW & GM & KM4 & KM5 & AS & TPHKB & TPGM & TPKM5 & TPAS & LIU \\
\hline 0.1 & 0.0084228 & 0.0081829 & 0.0084165 & 0.0274848 & 0.0078995 & 0.0057491 & 0.0079422 & 0.0050875 & 0.0068132 & 0.0054223 & 0.005091 & 0.0029807 \\
\hline 0.5 & 0.2105689 & 0.142061 & 0.206779 & 0.1723412 & 0.199381 & 0.1195448 & 0.1870799 & 0.1128457 & 0.1145735 & 0.1081953 & 0.1182638 & 0.0733781 \\
\hline 1 & 0.8422755 & 0.4552908 & 0.7944422 & 0.589147 & 0.8000254 & 0.4683981 & 0.6451712 & 0.4240638 & 0.4147371 & 0.4231201 & 0.4589172 & 0.2921224 \\
\hline 4 & 13.476407 & 6.99257 & 11.664827 & 9.308667 & 12.874578 & 7.446916 & 7.690046 & 6.539806 & 6.317763 & 6.739574 & 6.823498 & 4.717 \\
\hline 10 & 84.22755 & 44.43721 & 72.14139 & 62.51251 & 80.83463 & 46.97573 & 49.31967 & 41.27454 & 39.78072 & 42.27507 & 42.78561 & 29.50965 \\
\hline
\end{tabular}

Table 4.13: Estimated MSE with $n=50, p=10, \gamma=0.9$

\begin{tabular}{|c|c|c|c|c|c|c|c|c|c|c|c|c|}
\hline sigma & LS & HKB & LW & GM & KM4 & KM5 & AS & TPHKB & TPGM & TPKM5 & TPAS & LIU \\
\hline 0.1 & 0.0171385 & 0.0160878 & 0.0171032 & 0.0171974 & 0.0144543 & 0.0098896 & 0.0137651 & 0.009742 & 0.0101154 & 0.0089067 & 0.0098869 & 0.0059323 \\
\hline 0.5 & 0.4284616 & 0.2488431 & 0.4064802 & 0.2882771 & 0.3689336 & 0.2365009 & 0.3186997 & 0.2197035 & 0.2028688 & 0.2086741 & 0.2403167 & 0.1475976 \\
\hline 1 & 1.7138465 & 0.8863237 & 1.4985533 & 1.1480594 & 1.4888807 & 0.9410616 & 1.094845 & 0.8323048 & 0.7914599 & 0.8283718 & 0.920826 & 0.5886096 \\
\hline 4 & 27.421545 & 14.219395 & 21.991581 & 19.189275 & 24.204195 & 15.180668 & 15.447081 & 12.998545 & 12.501353 & 13.132833 & 13.694628 & 9.434745 \\
\hline 10 & 171.3846 & 90.22121 & 137.76715 & 126.50171 & 152.86653 & 96.26811 & 98.91006 & 82.2925 & 79.04653 & 82.74551 & 86.72064 & 59.44376 \\
\hline
\end{tabular}


Table 4.14: Estimated MSE, average $k$, s.d. of $k$ with $n=30, p=6, y=0.7$

\begin{tabular}{|c|c|c|c|c|c|c|c|c|c|c|c|c|}
\hline sigma & LS & HKB & LW & GM & KM4 & KM5 & AS & LIU & TPHKB & TPGM & TPKM5 & TPAS \\
\hline \multirow[t]{3}{*}{0.1} & 0.005169 & 0.00509 & 0.005165 & 0.014476 & 0.004765 & 0.003741 & 0.004956 & 0.001869 & 0.003189 & 0.004116 & 0.003537 & 0.003174 \\
\hline & & $\begin{array}{c}(0.059663 \\
0.001006)\end{array}$ & $\begin{array}{c}(0.002583 \\
0.000325)\end{array}$ & $\begin{array}{c}(14.70597 \\
19.41113)\end{array}$ & $\begin{array}{c}(0.343651 \\
0.12918)\end{array}$ & $\begin{array}{c}(3.441961 \\
1.691243)\end{array}$ & $\begin{array}{c}(0.096147 \\
0.062497)\end{array}$ & & & & & \\
\hline & & 100 & 100 & 41.1 & 100 & 97.2 & 100 & 100 & 100 & 100 & 100 & 100 \\
\hline \multirow[t]{3}{*}{0.5} & 0.129224 & 0.09813 & 0.127123 & 0.097298 & 0.121113 & 0.075808 & 0.117125 & 0.047013 & 0.066782 & 0.071607 & 0.065659 & 0.067821 \\
\hline & & $\begin{array}{c}(1.3326 \\
0.142297)\end{array}$ & $\begin{array}{l}(0.063327 \\
0.027755)\end{array}$ & $\begin{array}{l}(24.80631 \\
37.68962)\end{array}$ & $\begin{array}{l}(0.264596 \\
0.098772)\end{array}$ & $\begin{array}{l}(4.461042 \\
2.215371)\end{array}$ & $\begin{array}{c}(0.337185 \\
0.06573)\end{array}$ & & & & & \\
\hline & & 100 & 100 & 82.8 & 100 & 99 & 100 & 100 & 100 & 100 & 100 & 100 \\
\hline \multirow[t]{3}{*}{1} & 0.516895 & 0.295662 & 0.492707 & 0.332272 & 0.487246 & 0.293787 & 0.404687 & 0.188729 & 0.255735 & 0.259074 & 0.254466 & 0.264526 \\
\hline & & $\begin{array}{c}(4.117401, \\
1.002566)\end{array}$ & $\begin{array}{l}(0.191533 \\
0.114045)\end{array}$ & $\begin{array}{l}(30.11345 \\
37.27834)\end{array}$ & $\begin{array}{l}(0.239132 \\
0.090408)\end{array}$ & $\begin{array}{l}(4.946125 \\
2.377419)\end{array}$ & $\begin{array}{l}(1.099273 \\
0.184969)\end{array}$ & & & & & \\
\hline & & 99.95 & 100 & 88.05 & 100 & 98.8 & 99.95 & 100 & 100 & 100 & 100 & 100 \\
\hline \multirow[t]{3}{*}{4} & 8.27032 & 4.045102 & 7.274132 & 5.384369 & 7.898347 & 4.480938 & 4.5821 & 2.9468 & 3.880841 & 3.821265 & 3.959094 & 3.939506 \\
\hline & & $\begin{array}{c}(14.84304 \\
10.75586)\end{array}$ & $\begin{array}{c}(0.601523 \\
0.67383)\end{array}$ & $\begin{array}{l}(52.90355 \\
79.00465)\end{array}$ & $\begin{array}{l}(0.183814 \\
0.071468)\end{array}$ & $\begin{array}{l}\text { (6.487171) } \\
3.290223)\end{array}$ & $\begin{array}{l}(11.13381 \\
16.35435)\end{array}$ & & & & & \\
\hline & & 99.55 & 100 & 84.45 & 100 & 97.9 & 96 & 100 & 100 & 100 & 100 & 100 \\
\hline \multirow[t]{3}{*}{10} & 51.6895 & 25.58743 & 44.88862 & 36.46683 & 49.68654 & 27.78412 & 29.80301 & 18.48397 & 24.38181 & 23.86778 & 24.79131 & 24.7117 \\
\hline & & $\begin{array}{l}(18.33704 \\
18.98134)\end{array}$ & $\begin{array}{c}(0.714358 \\
1.055777)\end{array}$ & $\begin{array}{c}(79.27496 \\
125.5558)\end{array}$ & $\begin{array}{l}(0.156943 \\
0.064798)\end{array}$ & $\begin{array}{l}(7.803691 \\
4.287955)\end{array}$ & $\begin{array}{c}(370.7379 \\
10728.73)\end{array}$ & & & & & \\
\hline & & 99.25 & 100 & 80.45 & 100 & 97.25 & 93.85 & 100 & 100 & 100 & 100 & 100 \\
\hline
\end{tabular}


Table 4.15: Estimated MSE, average $k$, s.d. of $k$ with $n=30, p=6, y=0.8$

\begin{tabular}{|c|c|c|c|c|c|c|c|c|c|c|c|c|}
\hline sigma & LS & HKB & LW & GM & KM4 & KM5 & AS & LIU & TPHKB & TPGM & TPKM5 & TPAS \\
\hline \multirow[t]{3}{*}{0.1} & 0.00772 & 0.007536 & 0.007712 & 0.009061 & 0.006696 & 0.00475 & 0.007009 & 0.002767 & 0.004173 & 0.005031 & 0.004325 & 0.004178 \\
\hline & & $\begin{array}{l}\text { (0.05951, } \\
0.000983)\end{array}$ & $\begin{array}{l}(0.00245 \\
0.000458)\end{array}$ & $\begin{array}{l}\text { (10.0616, } \\
11.87183)\end{array}$ & $\begin{array}{l}\text { (0.40939, } \\
0.153969)\end{array}$ & $\begin{array}{l}(2.87419 \\
1.342212)\end{array}$ & $\begin{array}{l}(0.13867 \\
0.100266)\end{array}$ & & & & & \\
\hline & & 100 & 100 & 72.75 & 100 & 98.55 & 100 & 100 & 100 & 100 & 100 & 100 \\
\hline \multirow[t]{3}{*}{0.5} & 0.193005 & 0.133358 & 0.187882 & 0.124091 & 0.17234 & 0.107451 & 0.162221 & 0.069253 & 0.097041 & 0.095998 & 0.093982 & 0.099737 \\
\hline & & $\begin{array}{c}(1.26493 \\
0.16284)\end{array}$ & $\begin{array}{l}(0.06727 \\
0.041156)\end{array}$ & $\begin{array}{l}\text { (18.0407, } \\
25.77398)\end{array}$ & $\begin{array}{l}\text { (0.31117, } \\
0.116853)\end{array}$ & $\begin{array}{l}\text { (3.80373, } \\
1.890526)\end{array}$ & $\begin{array}{l}(0.37912 \\
0.102015)\end{array}$ & & & & & \\
\hline & & 99.95 & 100 & 87.4 & 99.95 & 97.4 & 99.95 & 100 & 100 & 100 & 100 & 100 \\
\hline \multirow[t]{3}{*}{1} & 0.772019 & 0.407981 & 0.714584 & 0.469636 & 0.697078 & 0.414901 & 0.54007 & 0.27583 & 0.371452 & 0.364961 & 0.369513 & 0.388307 \\
\hline & & $\begin{array}{l}\text { (3.64658, } \\
\text { 1.006989) }\end{array}$ & $\begin{array}{l}(0.20708 \\
0.157627)\end{array}$ & $\begin{array}{l}(23.5827 \\
40.22962)\end{array}$ & $\begin{array}{l}(0.27566 \\
0.103902)\end{array}$ & $\begin{array}{l}(4.31292 \\
2.232464)\end{array}$ & $\begin{array}{l}\text { (1.13507, } \\
0.198965)\end{array}$ & & & & & \\
\hline & & 99.95 & 100 & 89.2 & 100 & 97.85 & 99.95 & 100 & 100 & 100 & 100 & 100 \\
\hline \multirow[t]{3}{*}{4} & 12.3523 & 6.110453 & 10.53628 & 8.276557 & 11.376 & 6.602244 & 6.945109 & 4.428472 & 5.807665 & 5.700715 & 5.889751 & 5.906828 \\
\hline & & $\begin{array}{l}\text { (10.8656, } \\
9.563891)\end{array}$ & $\begin{array}{l}\text { (0.53368, } \\
0.722205)\end{array}$ & $\begin{array}{l}(38.2894 \\
58.54542)\end{array}$ & $\begin{array}{l}\text { (0.21817 } \\
0.085112)\end{array}$ & $\begin{array}{l}(5.49570 \\
2.844425)\end{array}$ & $\begin{array}{l}\text { (8.55986, } \\
11.48202)\end{array}$ & & & & & \\
\hline & & 98.65 & 100 & 83.7 & 100 & 96.2 & 94.8 & 100 & 100 & 100 & 100 & 100 \\
\hline \multirow[t]{3}{*}{10} & 77.20187 & 38.19354 & 65.3593 & 55.4644 & 71.99711 & 41.06761 & 44.07596 & 27.38699 & 36.07819 & 35.35801 & 36.43697 & 36.63483 \\
\hline & & $\begin{array}{c}(12.5978 \\
13.1616)\end{array}$ & $\begin{array}{l}\text { (0.59567, } \\
1.017023)\end{array}$ & $\begin{array}{c}(63.3459 \\
144.2615)\end{array}$ & $\begin{array}{l}\text { (0.18281, } \\
0.077798)\end{array}$ & $\begin{array}{l}(6.80775 \\
4.124177)\end{array}$ & $\begin{array}{l}(373.032 \\
11347.91)\end{array}$ & & & & & \\
\hline & & 98.75 & 100 & 79.75 & 100 & 94.95 & 94.65 & 100 & 100 & 100 & 100 & 100 \\
\hline
\end{tabular}


Table 4.16: Estimated MSE, average $k$, s.d. of $k$ with $n=30, p=6, y=0.9$

\begin{tabular}{|c|c|c|c|c|c|c|c|c|c|c|c|c|}
\hline sigma & LS & HKB & LW & GM & KM4 & KM5 & AS & LIU & TPHKB & TPGM & TPKM5 & TPAS \\
\hline \multirow[t]{3}{*}{0.1} & 0.015896 & 0.015083 & 0.015862 & 0.010644 & 0.01159 & 0.008824 & 0.011565 & 0.005737 & 0.008379 & 0.008221 & 0.007861 & 0.008528 \\
\hline & & $\begin{array}{l}(0.05903 \\
0.001088)\end{array}$ & $\begin{array}{c}(0.002302 \\
0.000718)\end{array}$ & $\begin{array}{l}(5.94673 \\
8.652064)\end{array}$ & $\begin{array}{l}(0.54461 \\
0.203563)\end{array}$ & $\begin{array}{l}(2.17560 \\
1.101852)\end{array}$ & $\begin{array}{l}(0.27493 \\
0.229402)\end{array}$ & & & & & \\
\hline & & 100 & 100 & 88.9 & 99.35 & 95.7 & 98.95 & 100 & 100 & 100 & 100 & 99.9 \\
\hline \multirow[t]{3}{*}{0.5} & 0.397398 & 0.232756 & 0.371915 & 0.245112 & 0.30433 & 0.216285 & 0.266974 & 0.142213 & 0.196652 & 0.186199 & 0.189202 & 0.208166 \\
\hline & & $\begin{array}{l}(1.10031, \\
0.209141)\end{array}$ & $\begin{array}{l}(0.08144 \\
0.075145)\end{array}$ & $\begin{array}{l}(10.2829 \\
15.64252)\end{array}$ & $\begin{array}{l}(0.41195 \\
0.153058)\end{array}$ & $\begin{array}{l}(2.87138 \\
1.427968)\end{array}$ & $\begin{array}{l}(0.51559, \\
0.230153)\end{array}$ & & & & & \\
\hline & & 99.9 & 100 & 87.05 & 99.8 & 93.75 & 97.95 & 100 & 100 & 100 & 100 & 99.95 \\
\hline \multirow[t]{3}{*}{1} & 1.589593 & 0.807853 & 1.378215 & 1.022296 & 1.24303 & 0.87692 & 0.940344 & 0.573312 & 0.761482 & 0.737442 & 0.75578 & 0.80943 \\
\hline & & $\begin{array}{l}(2.66120 \\
1.075268)\end{array}$ & $\begin{array}{l}\text { (0.20777) } \\
0.228632)\end{array}$ & $\begin{array}{l}(12.8825 \\
19.27105)\end{array}$ & $\begin{array}{c}(0.370784 \\
0.139741)\end{array}$ & $\begin{array}{l}(3.20172 \\
1.622612)\end{array}$ & $\begin{array}{c}(1.19422 \\
0.332469)\end{array}$ & & & & & \\
\hline & & 98.55 & 100 & 844.5 & 99.85 & 93.05 & 96.55 & 100 & 100 & 100 & 100 & 100 \\
\hline \multirow[t]{3}{*}{4} & 25.43348 & 12.71991 & 20.56304 & 17.82145 & 20.68535 & 14.16653 & 14.61344 & 9.070651 & 11.82114 & 11.54778 & 11.83807 & 12.14534 \\
\hline & & $\begin{array}{l}(5.99370 \\
6.254837)\end{array}$ & $\begin{array}{l}(0.42112 \\
0.738302)\end{array}$ & $\begin{array}{l}(21.6235 \\
44.11132)\end{array}$ & $\begin{array}{l}(0.29274 \\
0.110116)\end{array}$ & $\begin{array}{l}(4.07913 \\
2.233101)\end{array}$ & $\begin{array}{l}\text { (6.03878, } \\
8.509219)\end{array}$ & & & & & \\
\hline & & 96.95 & 100 & 79.55 & 99.95 & 90.2 & 91.45 & 100 & 100 & 100 & 100 & 100 \\
\hline \multirow[t]{3}{*}{10} & 158.9593 & 78.41805 & 126.2277 & 117.4674 & 133.3351 & 89.05708 & 91.10128 & 55.44889 & 72.71819 & 70.7814 & 72.40383 & 74.72872 \\
\hline & & $\begin{array}{l}\text { (6.79058, } \\
8.346396)\end{array}$ & $\begin{array}{l}\text { (0.50858, } \\
1.167226)\end{array}$ & $\begin{array}{r}(34.0470 \\
47.7129)\end{array}$ & $\begin{array}{l}(0.23686 \\
0.096607)\end{array}$ & $\begin{array}{l}(5.15030 \\
2.743206)\end{array}$ & $\begin{array}{l}(4318.49 \\
181606.3)\end{array}$ & & & & & \\
\hline & & 97.2 & 100 & 77.75 & 99.95 & 89.55 & 91.5 & 100 & 100 & 100 & 100 & 99.95 \\
\hline
\end{tabular}




\subsection{Simulated Results.}

I will discuss the simulation results in this section. A comparison will be made among the estimators based on the smaller MSE criterion for different values of $p, k, d$ and $\rho$.

\subsubsection{Performance as a function of $\sigma$.}

From Tables 4.2-4.13, we can compare MSEs of the estimators as a function of the variance of the errors $\left(\sigma^{2}\right)$. When the value of $\sigma$ increases, the MSE of the estimators also increases. For all values of $\sigma$, the ridge regression estimators and the two parameter biased estimators have smaller MSE compared with the LSE. However, the performance of the two parameter biased estimators is better than the performance of the corresponding ridge regression estimators. I also observe that the MSE of Liu estimator is the smallest which is a special case of two parameter estimator for $d=0$. This behavior was almost constant for any sample size and number of variables considered.

Amongst ridge estimators, KM5 performs better than others estimators for $\sigma<1$, however for $\sigma>1$, HKB performs better than the rest of ridge estimators closely followed by KM5. But to note here, from Tables 4.14-4.16, we see from the percentages that the MSE of KM5 is not always less than that of LSE. There is decrease in the percentage times the MSE of KM5 outperform the MSE of LSE with increase in sigma. For $\sigma<1$, amongst $\mathrm{HKB}$ and $\mathrm{KM} 5$, better choice is that to choose HKB as the average $k$ and s.d of $k$ corresponding to HKB are smaller and thus it is more reliable. Mostly performance of all the estimator are between HKB and LW except that of KM4 for larger values of $\sigma$. All the two parameter biased estimators perform better than ridge estimators, TPGM performs better than rest of the two parameter biased estimators for $\sigma>1$ and for $\sigma<1$ 
TPKM5 performs better than the rest the two parameter biased estimators. Liu estimator out performs all the estimators in all cases.

For given $y=0.70$ and $n=15$, the performance of estimators as a function of the standard deviation of the errors for $p=4$ and $p=10$ are provided in Figures 4.1 and 4.2 respectively. From these figures I observe that as the standard deviation increases, the MSE also increases.

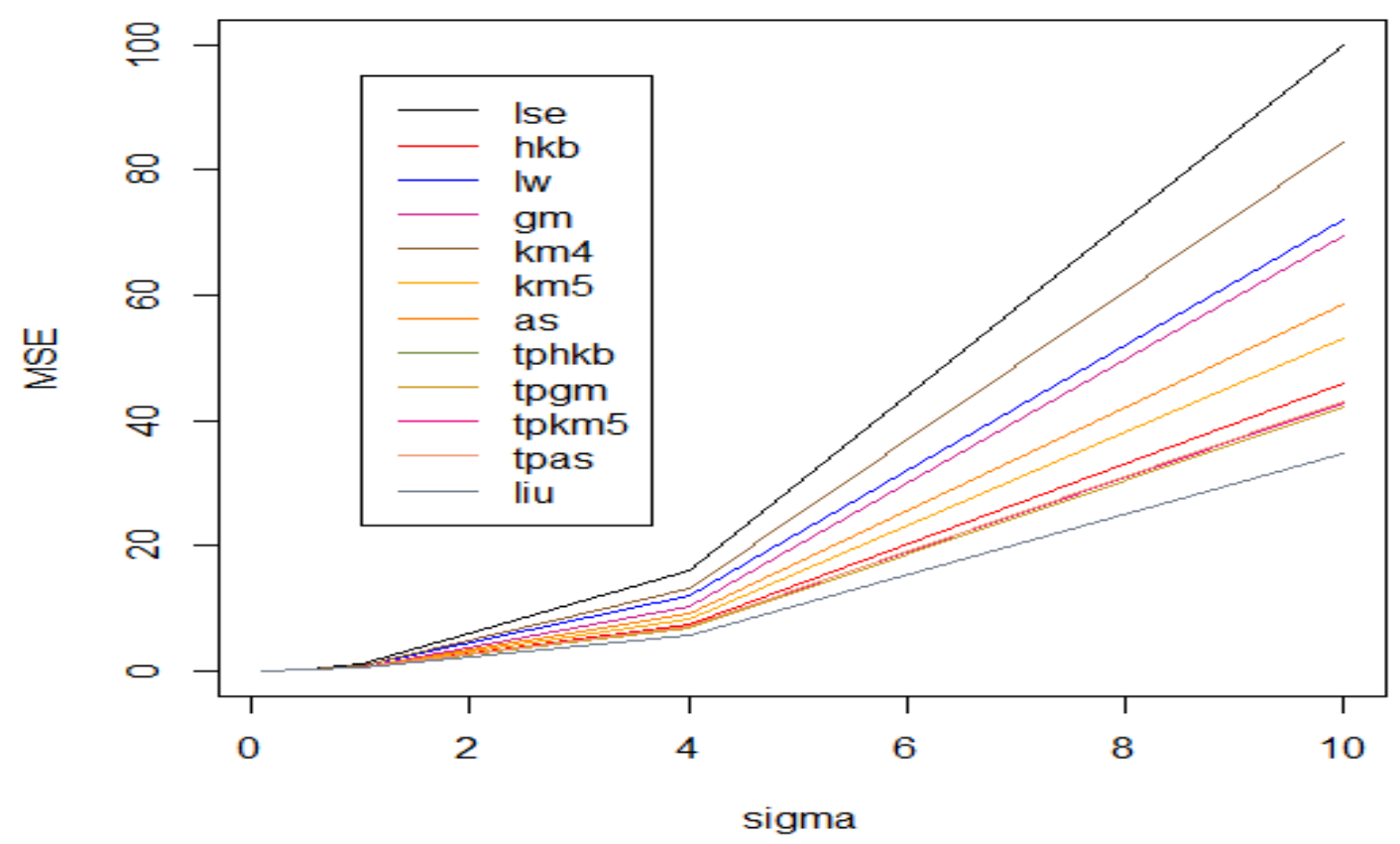

Figure 4.1: Performance of estimators as a function of $\sigma$, for $p=4, \gamma=0.70$ and $n=15$ 


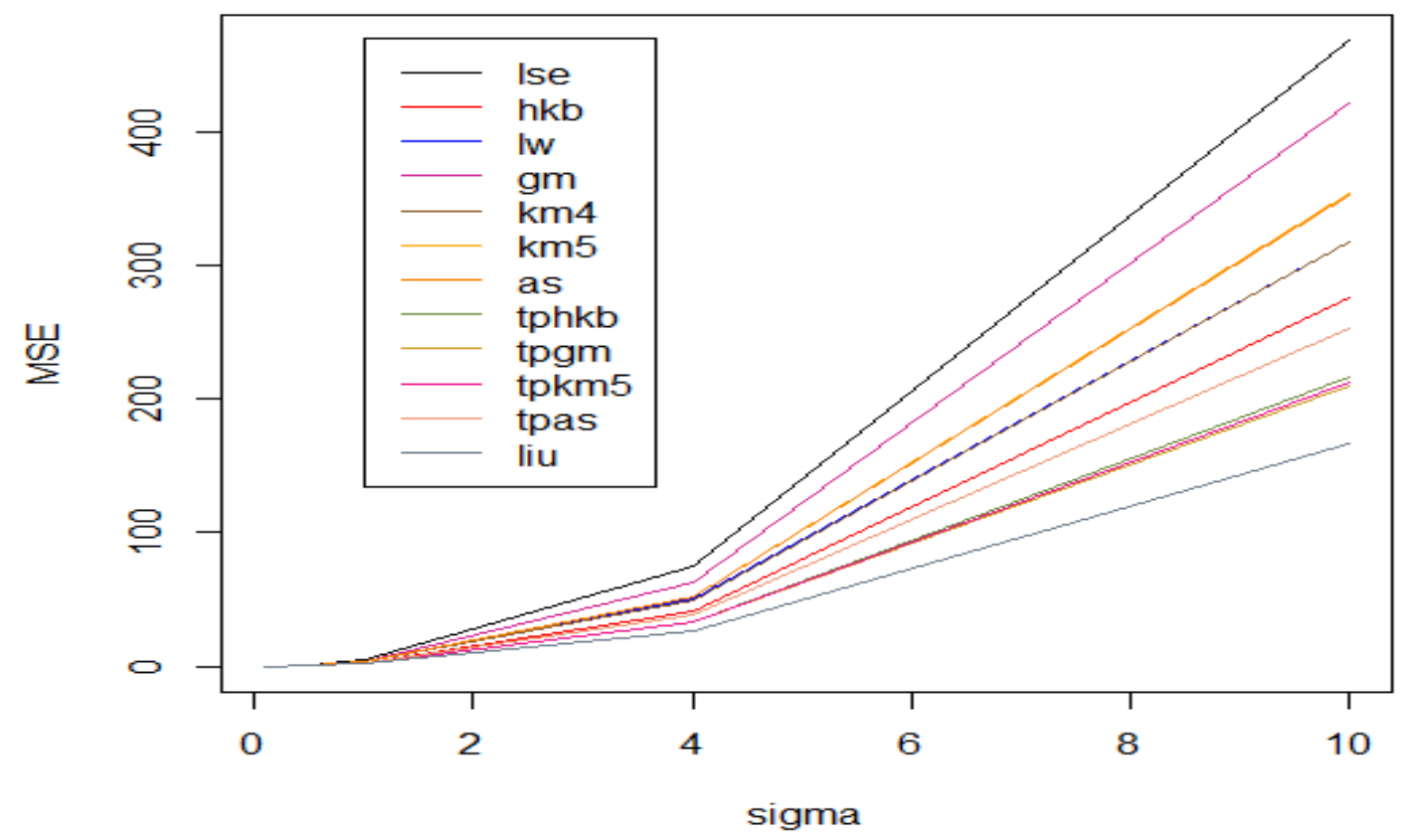

Figure 4.2: Performance of estimators as a function of $\sigma$, for $p=10, \gamma=0.70$ and $n=15$

\subsubsection{Performance as a function of $y$.}

From, Tables 4.2-4.13, I observe that for smaller sigma $(\sigma=0.01)$ the change in the correlation between the explanatory variables had almost no effect on the MSEs. In all situations they remained almost the same for any sample size or number of parameters, and their MSEs are very small. When $\sigma$ increases, the higher correlation between the independent variables, results in an increase of the MSE of the all estimators.

In general, all the two parameter biased estimators except TPAS perform better than rest of estimators other than KM4. For given $\sigma=1$ and $p=10$, the performance of estimators as a function of the correlation between the explanatory variables for $n=15$ and $n=50$ are provided in Figures 4.3 and 4.4 respectively. From these figures I observed that as correlation increases, the MSE also increases. All of the estimators 
have smaller MSE compared with LSE. Liu estimator again outperforms all other estimators, as special case of two parameter biased estimator. The MSEs of all the two parameter biased estimators are very close.

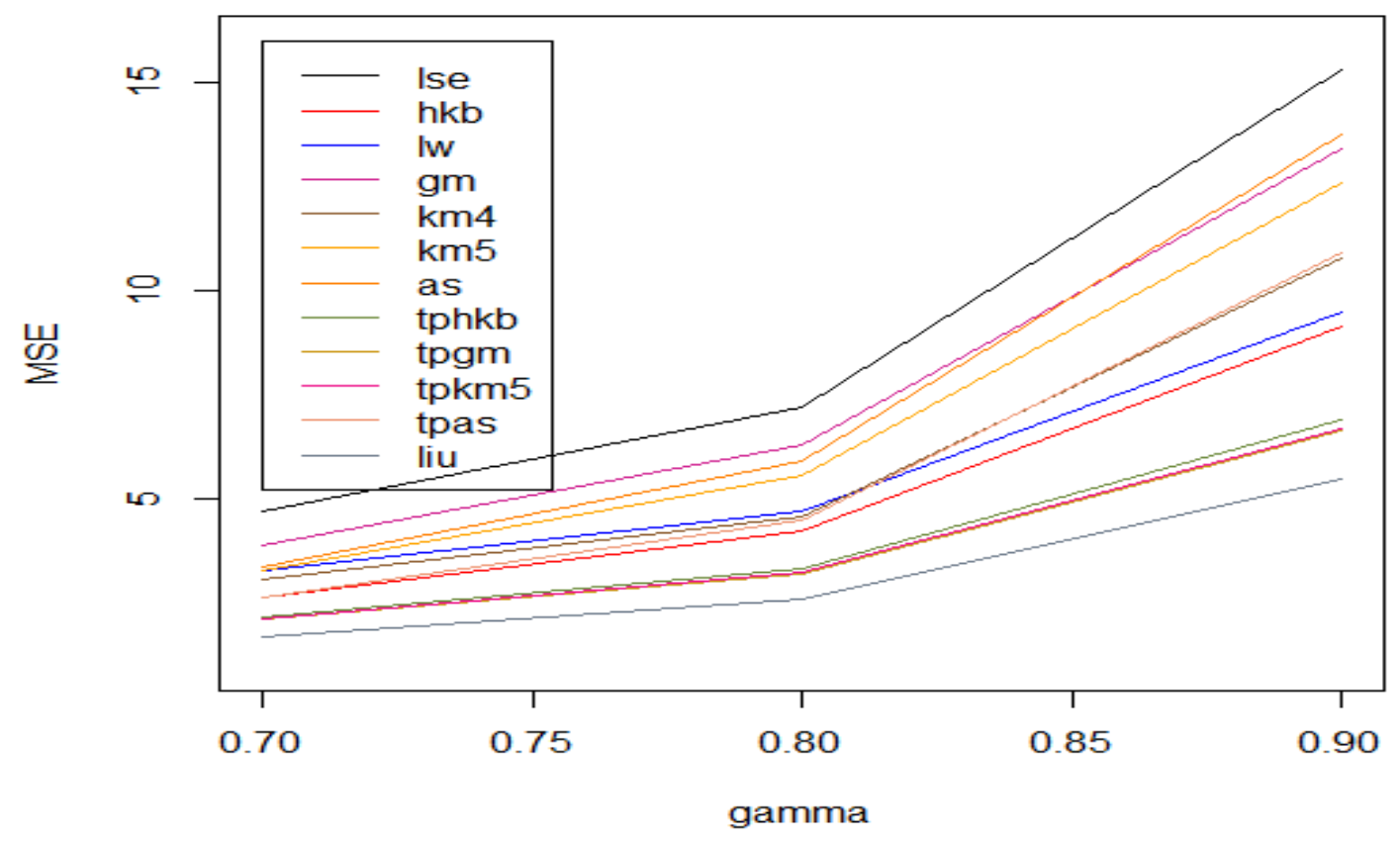

Figure 4.3: Performance of estimators as a function of $\gamma$, for $n=15$ 


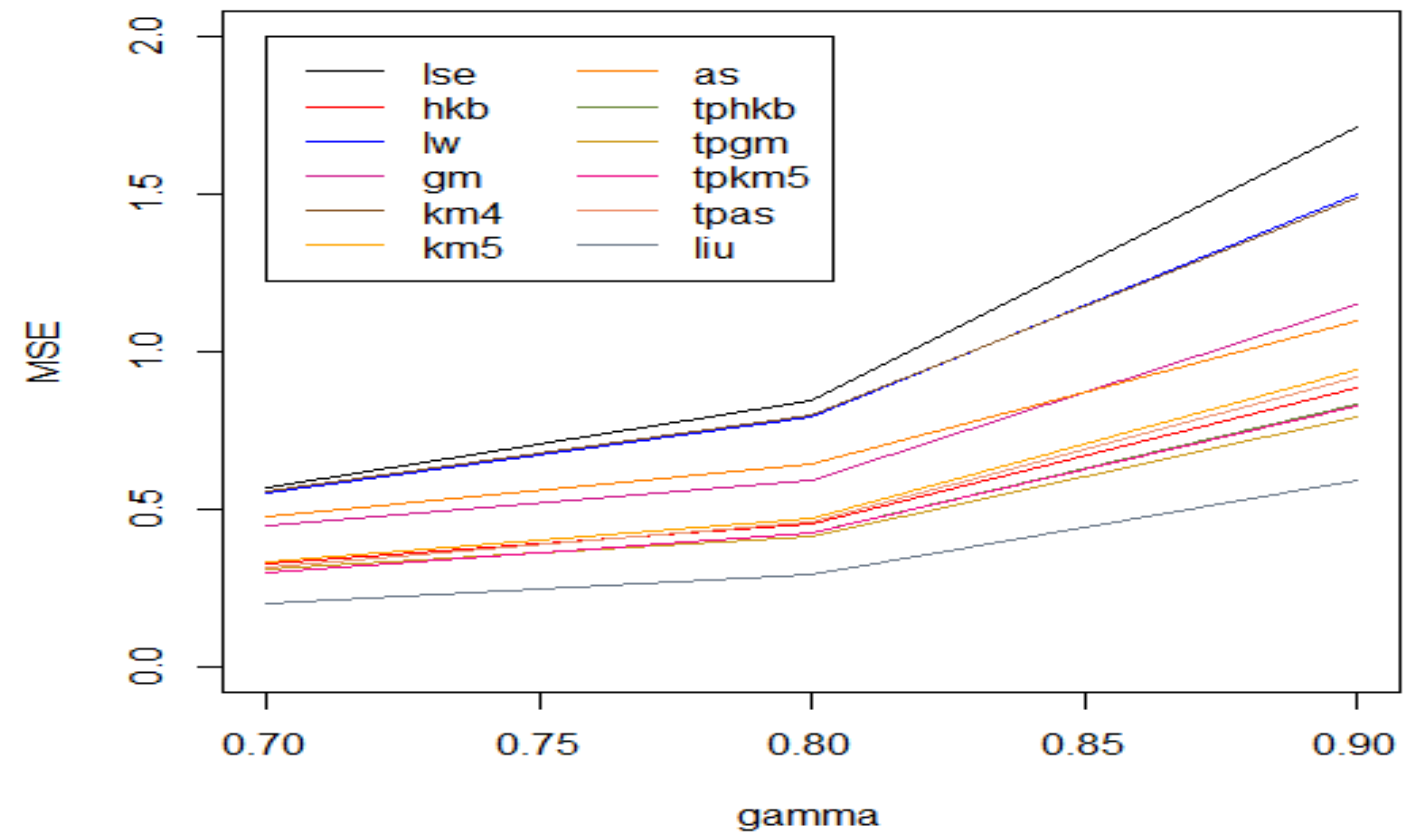

Figure 4.4: Performance of estimators as a function of $y$, for $n=50$

\subsubsection{Performance as a function of $\boldsymbol{n}$ and $\boldsymbol{p}$.}

From Tables 4.2 to 4.13 , I observed that, in general, when the sample size increases, the MSE decreases, or remained the same. Even for the large values of $\gamma$ and $\sigma$, if I increase the sample size, the MSE of estimators decrease. Again in this situation, as $n$ increases the performance of TPHKB, TPGM, TPKM5, TPAS and Liu better than the rest of the ridge estimators, also TPGM performs better amongst all the two parameter biased estimator and Liu performs better overall. From Tables 4.14 to 4.16 , we also observe that amongst all the Two parameter biased estimators TPHKB and TPKM5 perform equally good but the s.d of $k$ for TPKM5 is smaller than that of TPHKB for $\sigma>1$, thus TPKM5 is recommended to be used than TPHKB. 
For given $\sigma$ and $\gamma$, as the number of explanatory variables increase, the MSE of proposed estimator increases and the performance of estimators is similar to that when compared for different $\sigma$ s. 


\section{SUMMARY AND CONCLUDING REMARKS}

The purpose of this research is two fold. Firstly I made an analytical comparison of LASSO, ridge regression estimator, Liu estimator and least square estimator. For selected values of $k, p$ and $\Delta^{2}$, I compared the risks (MSE) and relative efficiencies and presented them in tabular form in Tables 3.1 to 3.12. I, also provided risk graphs for a visual comparison.

Based on the analyses of risks and relative efficiencies, I found that none of estimator uniformly dominate each other. I compared all the estimators for their dominance criteria in terms of $\Delta^{2}$, each criteria is found to be an increasing function of $p$. I found LSE mostly being dominated uniformly by rest of the estimators over a wider sub-space except for smaller values of $p$ at 3 and 4 . Also as value of $k$ increases the sub-space where ridge estimator is dominated by LASSO and Liu estimator, where $d$ equals $k$, increases. The results are similar for Liu estimator for decreasing values of $d$, at small value of $d$, LASSO and ridge estimator with $k$ equal to $d$ dominate it over a wider sub-space. This phenomenon increases for LASSO with smaller values of $q$, as $p$ increases. Neither estimator dominates one another uniformly except for LASSO at larger values of $p$ and small q. Finally, neither LASSO, ridge regression nor Liu estimator perform uniformly better than one other.

Secondly, I compared the Ridge regression, Liu, two parameter estimator and least square estimators under multicollinear model with error distribution being normal. The performance of the estimators depends on the variance of the random error, the correlations among the explanatory variables, the sample size and the unknown coefficients vectors. Based on the simulation study, some conclusions might be drawn. However, these conclusions might be restricted to the set of experimental conditions 
which are investigated. I used the MSE criteria to measure the goodness of the estimators. Increase in the value of $\sigma$ and the increase of the correlation between the independent variables have a negative effect on the MSE, in the sense that it also increases. When the sample size increases the MSE decreases, even when the correlation between the independent variables and $\sigma$ are large. The two parameter biased estimator gave better performance than the corresponding ridge regression estimator. Comparing the choice of $k, k_{\mathrm{GM}}$ performed better than rest of estimators for $\sigma$ $<1$ and $k_{\mathrm{KM} 5}$ performed better for $\sigma>1$. In conclusion, two parameter biased estimator with appropriate $k$ might be considered over ridge regression, as observed from simulated results. Finally, Liu estimator is a special case of two parameter biased estimator at $d=0$, outperforms every estimator. However, more study is required before making any definite statement.

For future researcher one may consider comparing the estimators under different error distribution, also the experiment was restricted to 2000 replications in the study which can be increased to attain more precise result in future. One variation that can be applied to the two parameter biased estimator is to replace $\tilde{\beta}$ by $\hat{\beta}_{\text {ridge }}$ instead of $\hat{\beta}_{L S}$. Also, lot of ridge estimators are available in the literature which can be considered and a comparison can be made amongst them. Variations such as the mean, geometric mean can be considered in estimating $d$, the shrinkage parameter for Liu estimator. 


\section{LIST OF REFERENCES}

Alkhamisi, M., Khalaf, G., Shukur, G. (2006). Some modifications for choosing ridge parameters Communications in Statistics- Theory and Methods, 35: 2005-2020.

Aslam, M. (2014). "Performance of Kibria's Method for the Heteroscedastic Ridge Regression Model: Some Monte Carlo Evidence". Communications in StatisticsSimulation and Computation, 43: 673-686.

Arashi, M. and Valizadeh, T. (2015).“Performance of Kibria's methods in partial linear ridge regression model". To appear in Statistical Papers.

Donoho, D. and Johnstone, I. (1994). "Ideal spatial adaption by wavelet shrinkage". Biometrika, 81, 425-455..

Dorugade, A. V. (2013). "New ridge parameters for ridge regression". Journal of the Association of Arab Universities for Basic and Applied Sciences. Vol.15. 94-99.

Galton, Sir Francis. (1885). "Regression towards mediocrity in heredity stature". Journal of Anthropological Institute 15: 246-263.

Gibbons, D.G. (1981). "A simulation study of some ridge estimators”. Journal of the American Statistical Association 76:131-139.

Golub, G. H., Health, M. and Wahaba, G. (1979). "Generalized cross-validation as a method for choosing a good ridge parameter". Technometrics, 21: 215-223.

Hefnawy, A. E and Farag, A. (2014). "A Combined Nonlinear Programming Model and Kibria Method for Choosing Ridge Parameter Regression". Communications in Statistics-Simulation and Computation, 43: 1442-1470, 2014

Hoerl, A.E., Kennard R.W. (1970). "Ridge regression: biased estimation for nonorthogonal problems". Technometrics 12: 55-67.

Hoerl, A.E., Kennard, R.W., Baldwin, K.F. (1975). "Ridge regression: some simulation". Communications in Statistics 4: 105-123

Khalaf, G. and Shukur, G. (2005). "Choosing ridge parameters for regression problems". Communication in Statistics- Theory and Methods, vol.34, pp.1177-1182.

Khalaf, G. (2012). "A proposed ridge parameter to improve the least squares estimator". Journal of Modern Applied Statistical Methods, vol.11, pp.443-449.

Kibria B. M. G. (2003). "Performance of some new ridge regression estimators". Communications in Statistics- Theory and Methods, 32:419-435.

Kibria, B. M. G. and Banik, S. (2015). "A Comprehensive Simulation Study on Some Ridge Regression Estimators and Their Performances". To appear in Journal of Modern Applied Statistical Methods. 
Lawless, J.F., Wang, P.(1976)." A simulation study of ridge and other regression estimators". Communications in Statistics A5: 307-323.

Liu, K. (1993). "A new class of biased estimate in linear regression". Communications in Statistics- Theory and Methods, Vol.22, No.2. 393-402

Liu, K. (2003). "Using Liu-Type Estimator to Combat Collinearity". Communications in Statistics - Theory and Methods, Vol.32, No.5. 1009-1020

McDonald, G.C., Galarneau D.I. (1975)." A Monte Carlo evaluation of some ridge-type estimators". Journal of the American Statistical Association 70:407-416.

Muniz, Gisela and Kibria, B.M. G. (2009). "On Some Ridge Regression Estimators : An Empirical Comparisons". Communications in Statistics- Theory and Methods, Vol.38, No.3. 621-630.

Mansson, K., Kibria, B.M. G., Shukur, G. (2012). "On Liu estimators for the logit regression model.” Economic Modelling. Vol.29 No.4., 1483-1488.

Newhouse, J. P. and Oman, S. D. 1971. "An evaluation of ridge estimators". Rand Corporation. P-716-PR

Saleh, A. K. Md. E. (2006). "Theory of Preliminary Test and Stein-Type Estimation with Applications". Wiley, New York, 2006.

Tibshirani, R. (1996). "Regression Shrinkage and Slection via the LASSO". Journal of the Royal Statistical Society. Series B(Methodological, Vol.58, No.1). 267-288. 